Florida International University

FIU Digital Commons

3-28-2019

\title{
Organizational Factors in Patient-Centered Medical Home Implementation and Chronic Disease Management in HRSA- Funded Community Health Centers
}

Willmarie Latorre

wlato001@fiu.edu

Follow this and additional works at: https://digitalcommons.fiu.edu/etd

Part of the Community Health and Preventive Medicine Commons, Health Services Administration Commons, Health Services Research Commons, and the Public Health Education and Promotion Commons

\section{Recommended Citation}

Latorre, Willmarie, "Organizational Factors in Patient-Centered Medical Home Implementation and Chronic Disease Management in HRSA-Funded Community Health Centers" (2019). FIU Electronic Theses and Dissertations. 4022.

https://digitalcommons.fiu.edu/etd/4022

This work is brought to you for free and open access by the University Graduate School at FIU Digital Commons. It has been accepted for inclusion in FIU Electronic Theses and Dissertations by an authorized administrator of FIU Digital Commons. For more information, please contact dcc@fiu.edu. 


\title{
FLORIDA INTERNATIONAL UNIVERSITY
}

\author{
Miami, Florida
}

\section{ORGANIZATIONAL FACTORS IN PATIENT-CENTERED MEDICAL HOME IMPLEMENTATION AND CHRONIC DISEASE MANAGEMENT IN HRSA- FUNDED COMMUNITY HEALTH CENTERS}

A dissertation submitted in partial fulfillment of

the requirements for the degree of

DOCTOR OF PHILOSOPHY

in

PUBLIC HEALTH

by

Willmarie Latorre 
To: Dean Tomás R. Guilarte

Robert Stempel College of Public Health and Social Work

This dissertation, written by Willmarie Latorre, and entitled Organizational Factors in Patient-Centered Medical Home Implementation and Chronic Disease Management in HRSA-Funded Community Health Centers, having been approved in respect to style and intellectual content, is referred to you for judgment.

We have read this dissertation and recommend that it be approved.

$\begin{array}{r}\text { William Darrow } \\ \hline \text { Patria Rojas } \\ \hline \text { Elena Bastida, Major Professor Campa }\end{array}$

Date of Defense: March 28, 2019

The dissertation of Willmarie Latorre is approved.

Dean Tomás R. Guilarte Robert Stempel College of Public Health and Social Work

Andrés G. Gil Vice President for Research and Economic Development and Dean of the University Graduate School

Florida International University, 2019 
(C) Copyright 2019 by Willmarie Latorre

All rights reserved. 


\section{DEDICATION}

I want to dedicate the product of my work to God. I am immensely grateful to you. Thank you for giving me the health and the strength to complete this journey.

I also dedicate this dissertation to my amazing family. Thank you for your patience and your loving support. Thanks for all the words of encouragement and for always believing in me.

"There is freedom waiting for you, On the breezes of the sky, and you ask: What if I fall? Oh but my darling, what if you fly?" - Erin Hanson 


\section{ACKNOWLEDGMENTS}

First and foremost, I would like to thank my Major Professor, Dr. Elena Bastida, for her persistent guidance and her faith in me. Dr. Bastida, I will be eternally grateful for your patience, your constant encouragement, and your mentorship. I have learned so much from you and feel privileged to have had the opportunity to work side-by-side with you in this process. To the members of my Committee, Dr. William Darrow, Dr. Patria Rojas, and Dr. Adriana Campa, it has been an honor to have you on my Dissertation Committee. I sincerely appreciate your invaluable guidance. Thank you for all the time you dedicated to this work and for helping me achieve this goal. Finally, I thank my family for being my rock all through this process, for your understanding, your patience, and unconditional love. 


\author{
ABSTRACT OF THE DISSERTATION \\ ORGANIZATIONAL FACTORS IN PATIENT-CENTERED MEDICAL HOME \\ IMPLEMENTATION AND CHRONIC DISEASE MANAGEMENT IN HRSA- \\ FUNDED COMMUNITY HEALTH CENTERS \\ by
}

Willmarie Latorre

Florida International University, 2019

Miami, Florida

Professor Elena Bastida, Major Professor

The Patient-Centered Medical Home (PCMH) model has attracted the attention of numerous health organizations in the United States for its potential to enhance quality of care and improve health outcomes among those living with chronic diseases. Community Health Centers (CHCs) funded by HRSA have been implementing this model for several years. Nevertheless, not all have achieved anticipated improvements. Several researchers have suggested that organizational factors may have a more determining role than the actual implementation of the PCMH model.

This qualitative case study explored organizational factors that distinguished or affected PCMH implementation at two CHCs with PCMH status recognized by HRSA as high-quality leaders. Three objectives were proposed: 1) compare and contrast organizational factors identified in the two CHCs; 2) describe how these CHCs implemented PCMH elements related to chronic disease management; and 3) propose an organizational framework to support PMCH implementation among CHCs. Three data 
collection procedures were employed: field observations, document review/analysis, and interviews.

Findings highlight the influence of organizational structure and organizational culture on PCMH implementation and chronic disease management initiatives. It also heightens the impact of multilevel efforts on the implementation of a complex organizational model like the PCMH. Other identified facilitators include the establishment of a "patient-centered" culture, active engagement of top-level leadership, and availability of fully-functioning health care teams supporting care coordination and implementation of the model across the organization. Findings also highlighted structural factors affecting PCMH implementation such as changes from one $\mathrm{PCMH}$-accrediting agency to another, which can destabilize PCMH implementation; the choice of an individual site instead of an organizational-level PCMH implementation, which can lead to lack of commitment among sites not recognized; and the high level of complexity of a multiple-site implementation.

This study is expected to contribute to the work of CHCs, an essential component of the US health system playing a key role in helping accomplish the nation's health goals. Given their role in the fight against chronic diseases, public health practitioners should pay attention to how well these organizations are moving toward the accomplishment of their mission and examine factors that can improve their performance in this endeavor. 


\section{TABLE OF CONTENTS}

I. SIGNIFICANCE AND LITERATURE REVIEW 14

The Impact of Chronic Diseases in Communities Served by CHCs 15

Chronic Disease Management: A Fundamental Strategy for CHCs 16

PCMH: An Organizational, Integrated Approach to Chronic Disease Management 20

$\begin{array}{ll}\text { PCMH Principles and Standards } & 21\end{array}$

Effectiveness of the Implementation of the PCMH Model on Chronic Disease

Outcomes $\quad 25$

Challenges in Studying the Impact of the PCMH model 29

Importance of Organizational Context and Dynamics in the Success of the PCMH 33

The PCMH within the Context of HRSA-Funded CHCs 36

Summary of Evidence and Research Gap 37

Summary of Challenges and Factors Involved in PCMH Implementation 40

II. METHODS, DATA COLLECTION, AND ANALYSIS PROCEDURES 43

Methods $\quad 44$

Overall Research Strategy $\quad 44$

Case Study Approach $\quad 45$

Setting and Unit of Study. $\quad 46$

Sampling and Recruitment. 48

Data Collection Procedures $\quad 51$

Non-Participant Direct Observations $\quad 51$

Document Review and Analysis $\quad 53$

Semi-Structured Interviews $\quad 55$

Member Checking/Validation. $\quad 57$

Data Analysis $\quad 57$

$\begin{array}{ll}\text { Data Interpretation } & 60\end{array}$

Quality of the Study and Ethical Considerations 61

Strategies to Enhance Quality and Rigor of the Study 61

$\begin{array}{ll}\text { Ethical Considerations } & 62\end{array}$

III. CASE 1

Community Health Center 1 (CHC1) Overview $\quad 70$

$\begin{array}{ll}\text { Site Description } & 71\end{array}$ 
$\begin{array}{ll}\text { Emerging Themes } & 72\end{array}$

$\begin{array}{ll}\text { Patient-Centered, Need-Based Care } & 73\end{array}$

Comprehensive and Integrated Care $\quad 73$

Patient Support and Enabling Services $\quad 75$

Linguistically and Culturally Appropriate Services $\quad 77$

Patient Participation in the Process of Care $\quad 78$

Access to Care $\quad 79$

Coordination and Integration of Care $\quad 81$

Care Coordination $\quad 81$

$\begin{array}{ll}\text { Planned Care } & 83\end{array}$

Team-Based Care $\quad 84$

Chronic Disease Management, Self-Care Support, and Education 85

Chronic Disease Management and Self-Care Support 85

Patient Communication and Education $\quad 88$

Use of Technology to Facilitate Patient Care and Population Health Management 90

Organizational System and Organizational Culture $\quad 92$

$\begin{array}{ll}\text { Organizational Structure } & 92\end{array}$

Organizational Culture, Leadership Culture, and Staff Characteristics 94

Learning Culture: Performance and Quality Improvement 96

$\begin{array}{ll}\text { Strategic Partnerships } & 97\end{array}$

Medical Home Implementation $\quad 98$

Approach to PCMH implementation $\quad 98$

Challenges and Barriers to Implementation $\quad 99$

$\begin{array}{ll}\text { PCMH Implementation Facilitators } & 100\end{array}$

Summary of Key Findings and Organizational Factors Identified 101

Salient Features of CHC1's PCMH Model 101

Summary of Challenges and Facilitators to PCMH Implementation 104

IV. CASE 2 106

Community Health Center 2 (CHC2) Overview 112

Site Description $\quad 113$

Emerging Themes $\quad 114$

$\begin{array}{ll}\text { Patient-Centered, Need-Based Care } & 115\end{array}$

Comprehensive and Integrated Care 115

$\begin{array}{ll}\text { Patient Support and Enabling Services } & 117\end{array}$

Culturally and Linguistically Appropriate Services 117

Patient and Family Participation in the Process of Care 119

$\begin{array}{ll}\text { Access to Care } & 120\end{array}$

Coordination and Integration of Care 121

$\begin{array}{ll}\text { Team-Based Care } & 121\end{array}$ 
Care Coordination $\quad 123$

Planned Care $\quad 124$

Education and Self-Care Support $\quad 126$

Patient Communication and Education 126

Chronic Disease Management and Self-Care Support 127

Organizational System and Organizational Culture 128

$\begin{array}{ll}\text { Organizational Structure } & 128\end{array}$

Organizational, Leadership, and Learning Culture $\quad 129$

Strategic Partnerships 131

Use of Technology to Facilitate Patient Care and Manage Population Health $\quad 131$

Medical Home Implementation 132

$\begin{array}{ll}\text { Approach to Patient-Centeredness } & 132\end{array}$

Approach to PCMH Implementation 133

Challenges and Barriers to Implementation 135

PCMH Implementation Facilitators 137

Summary of Key Findings and Organizational Factors Identified 138

Salient Features of CHC2's PCMH Model 139

Summary of Challenges and Facilitators to PCMH Implementation 141

V. DISCUSSION AND CONCLUSION 143

Summary of the Research 144

Discussion $\quad 146$

Organizational Factors $\quad 151$

Summary of Organizational Barriers and Facilitators 155

Challenges and Barriers to Implementation 155

PCMH Implementation Facilitators 157

Proposed Organizational Framework for PCMH Implementation at CHCs 159

Conclusions and Lessons Learned 160

Lessons Learned 161

$\begin{array}{ll}\text { REFERENCES } & 164\end{array}$

$\begin{array}{ll}\text { APPENDICES } & 173\end{array}$

$\begin{array}{ll}\text { VITA } & 212\end{array}$ 


\section{LIST OF TABLES}

TABLE

PAGE

1. Major factors and barriers to PCMH implementation identified in $\mathrm{PCMH}$ literature

2. Documents reviewed as part of the research process at $\mathrm{CHC} 1$

3. Case 1 Interview Guiding Questions and Emerging Themes

4. Categorized emerging themes for Case 1

5. Health services provided by $\mathrm{CHC} 1$

6. Summary of Challenges and Facilitators to PCMH Implementation at CHC1

7. Documents reviewed as part of the research process at $\mathrm{CHC} 2$

8. Case 2 Interview Guiding Questions and Emerging Themes

9. Categorized emerging themes for Case 2

11. Summary of Challenges and Facilitators to PCMH Implementation at $\mathrm{CHC} 2$

12. Summary of organizational barriers and facilitators to PCMH implementation at $\mathrm{CHCs}$ 


\section{LIST OF FIGURES}

FIGURE

PAGE

1. The Chronic Care Model

2. Illustration of the case study/ data analysis process

58

3. Proposed organizational framework for PCMH implementation at $\mathrm{CHCs}$

159 


\section{INTRODUCTION}

\section{Statement of the Problem}

The Patient-Centered Medical Home (PCMH) model has increasingly attracted the attention of health organizations in the United States as an approach with the potential to improve health outcomes among those with chronic disease, improve quality of care, and reduce health care costs (Martsolf et al., 2012). This health care model promotes the delivery of patient-centered, comprehensive, coordinated, accessible, and evidence-based care, placing emphasis on the whole person, his/her family, and his/her overall well-being (Beacham et al., 2012; Martsolf et al., 2012; Cook et al., 2015; Platonova et al., 2016). Community Health Centers (CHCs) funded by the Health Resources and Services Administration (HRSA), which serve more than 27 million individuals across the 50 states and US territories, have been implementing this model since 2011. Nevertheless, not all have achieved the anticipated improvements. As reported by the National Association of Community Health Centers (NACHC) (2017), key health outcomes such as diabetes and hypertension control are met by only $37 \%$ and $15 \%$ of $\mathrm{CHCs}$, respectively (NACHC, 2017). In 2017, 33\% of CHC patients with diabetes had HbA1c levels greater than $9 \%$ and more than $37 \%$ of patients with hypertension were uncontrolled (HRSA, 2018a).

Given the significant role of CHCs in the quality of life and well-being of those living in the most disadvantaged communities in the nation, the main driver behind the present research is to explore and characterize factors with potential influence on the capacity of these health organizations to implement this integrated model of care and 
bring about desired chronic disease outcomes among communities served. Researchers have found that both having PCMH status and implementing a few components of the PCMH model are associated with improved processes of care, certain health outcomes, and patient experience with care. However, as further elaborated in Chapter 1 of this dissertation, existing evidence on PCMH effectiveness to date seems to be inconsistent. One key challenge in studying the impact of this model is the substantial variability in the way the model is implemented, mostly due to its ambiguity and "conceptual sponginess," an issue further explained in Chapter 1 (Hoff et al., 2012).

Several authors have placed emphasis on the importance of understanding the existing difference between obtaining PCMH status and fully committing to PCMH implementation. $\mathrm{PCMH}$ recognition does not necessarily mean that an organization is actually using or implementing all the model's principles (Dobbins et al., 2018). While the PCMH model is constituted by a series of principles identified as best practices, once recognition is obtained, not every health organization implements the model completely. Variations in performance and results across health centers, according to Shippee et al. (2017), show differences in interventions, scope of implementation, and populations under study, as health centers address PCMH principles or standards differently. The PCMH does not follow a homogeneous design, but an adaptive approach (Shippee et al., 2017).

Current PCMH standards provide little guidance on how to strategically implement the model and how to adapt it to different organizational contexts (Hoff et al., 2012). In fact, multiple researchers have expressed concerns regarding whether different primary care practice settings, with different resources and constraints, should have 
similar PCMH interventions and outcome goals (Goldman et al., 2015; Quinn et al., 2013; and Hoff et al., 2012). It has been often suggested that organizational context plays a fundamental role and that the effectiveness of PCMH implementation depends on organizational factors, rather than on the actual implementation of the model. Hence, some stress the need of understanding why the PCMH may only work in some contexts and how organizational factors are involved in achieving PCMH outcomes (Goldman et al., 2015). This is highly relevant in understanding the outcomes of PCMH implementation at $\mathrm{CHCs}$, as they confront numerous organizational, financial, and community challenges in carrying out their mission of improving the lives of disadvantaged communities.

Successful implementation of the PCMH model could provide HRSA-funded CHCs with an invaluable opportunity to enhance their chronic disease management performance and, thus, improve health outcomes for those living with chronic diseases. There is, however, a need to understand why anticipated PCMH outcomes with regards to chronic disease management have not been equally achieved, why PCMH has only seemed to work effectively at certain $\mathrm{CHCs}$, and what differentiates successful models at CHCs. According to current research, which is discussed thoroughly in Chapter 1, variations in CHC performance, $\mathrm{PCMH}$ implementation, and chronic disease outcomes across CHCs nationwide may be the result of the influence of factors beyond PCMH principles and HRSA support, including organizational context.

\section{Study Aim and Objectives}

This study explores and describes the organizational factors that contribute to successful PCMH implementation and improvement of chronic disease management 
outcomes among HRSA-funded CHCs with PCMH recognition. For the purpose of this research, organizational factors include the organizational level aspects, characteristics, or dynamics that contribute to or hinder PCMH implementation and concomitant improvements in chronic disease management. The specific aim is to explore and characterize organizational factors that distinguish or affect PCMH implementation at two CHCs with PCMH status recognized by HRSA as high-quality leaders with variations in context. In exploring this aim, three objectives were proposed:

1) compare and contrast organizational factors identified in the two participating CHCs;

2) describe how these CHCs have implemented PCMH elements related to chronic disease management, mainly diabetes and hypertension control; and 3) use theoretically-framed interpretations to propose an organizational model to support chronic disease management and PMCH implementation among HRSAfunded CHCs.

\section{Overview of the Research Methods}

A qualitative collective case study was designed and implemented to support the accomplishment of the study's aim. The qualitative research strategy provided an opportunity to explore the phenomenon of interest within its natural setting, understand how it develops within the particular conditions of $\mathrm{CHCs}$, and examine the multiple factors and dimensions related to PCMH implementation and chronic disease management as they emerged. In addition, the case study approach allowed the study of the phenomenon through the use of multiple sources and perspectives. The information- 
rich cases selected allowed a comprehensive description and analysis of the phenomenon and the particularities of the two different contexts in which it developed.

The study employed a purposeful and theoretical sampling approach. Data collection took place within settings where the phenomenon occurs: HRSA-funded community health centers with PCMH recognition. Two CHCs with PCMH status were included as part of this qualitative collective case study. In addition to having PCMH recognition, at the time of the study, the two cases selected were recognized by HRSA as "Health Center Quality Leaders" for having the best overall performance among all CHCs in a series of chronic disease management and preventive care measures and had been previously recognized by HRSA as "National Quality Leaders" for meeting or exceeding national benchmarks. PCMH status and HRSA recognition were fundamental in expanding the research's potential of finding and exploring successful models of PCMH implementation at CHCs. Moreover, to document variations in the implementation, context and culture of the PCMH, the cases selected for the study included one case from a group of HRSA-funded CHCs in South Florida and a second case from a group of CHCs in the US Commonwealth of Puerto Rico.

The two cases were purposefully selected to reach maximum variation with regards to organizational context. The cases studied demonstrated optimal performance amid very different contexts and organizational cultures. This decision allowed the documentation of unique variations brought up by the conditions of each site. Moreover, this gave place to an opportunity to explore the organizational adaptation of the PCMH implementation. Existing differences in patient and staff mix, organizational cultures, behaviors, language use, patient-provider/staff interactions, and practices, among others, 
provided an instrumental opportunity to assess the implementation of the PCMH model from two distinctive perspectives.

Taking full advantage of the case study approach, the study employed three data collection methods: field observations, interviews, and documentation review/content analysis. The use of multiple data collection sources provided access to a comprehensive picture of the manifestation of the phenomenon of interest. Overall, data collection involved 70 hours of field observation, 35 at each site. Fifty-six (56) hours of observation took place in general public areas, patient waiting rooms, hallways, and general service areas. The remaining 14 hours included six (6) hours of observations at corporate and administrative facilities and a total of eight (8) hours observing community and location.

The second data collection phase included the review and analysis of PCMHrelated documents, announcements, and patient communications. Contents from multiple documents provided by both sites were thoroughly analyzed to identify common patterns and categories. Documents obtained were qualitatively analyzed to find significant meanings and themes and to establish links with the evidence obtained from observations and interviews. Documents reviewed included: PCMH meeting minutes; program related policies; documentation of compliance with PCMH standards; documentation of accomplishments in key PCMH/chronic disease objectives; quality of care performance measures; quality/performance improvement documents; sample documents submitted to accrediting agencies (e.g., NCQA); sample of patient record review worksheets; showing tracking of compliance with PCMH standards; organizational charts; and announcements or communication pieces. 
Other data collected included publicly-available key health center quantitative measures such as: patients' race/ ethnicity (proportions by category); language (percentage of patients best served in a language other than English); percentage of patients living below poverty; percentage of uninsured patients and Medicaid and Medicare beneficiaries; percentage of homeless individuals and public housing residents served; percentage of older adults served; percentage of patients served with hypertension and diabetes, respectively; percentage of patients with uncontrolled diabetes; and percentage of patients with uncontrolled hypertension.

A series of semi-structured interviews constituted the last data collection phase of the study. These conversations were a central piece in the case development process, as they were vital in understanding the reasons behind current PCMH implementation practices and outcomes. There were close to 13 hours of interviews, over six at the first site and six at the second site, with a total of 13 health center staff members. Interview duration ranged from 15 to 120 minutes. Interviewees included health center leaders, health care providers, medical home coordinators, quality improvement personnel, and allied health professionals. Interviews were transcribed verbatim and analyzed using NVivo 12 as a tool to facilitate data organization and identification of emerging themes and patterns.

After completing data collection at each site, a preliminary within-case analysis process was conducted in order to provide space for validation and corroboration of findings and obtain further input from key informants. Preliminary data were shared with key health center personnel as part of a member-checking process that provided another 
means of corroboration and validation of findings. Input received during this participatory process was anonymously incorporated into findings.

As further explained in Chapter 2, data analysis took place across four major phases: 1) preparation; 2) development of individual case and context description; 3) within-case analysis; and 4) cross-case analysis. Data collected allowed the development of two individual cases that characterize and illustrate the contexts of both participating organizations, their organizational cultures, key internal actors, PCMH implementation practices, and chronic disease management strategies. Both cases were then crossanalyzed to fulfill the overall purpose of the study. This cross-case analysis and both the emerging and theoretically-framed interpretations led to the development of a framework for the development of contextually-relevant PCMH interventions that support comprehensive chronic disease management at CHCs. Chapter 2 provides full details on the research strategy selected, study settings, data collection methods, and data analysis procedures.

\section{Theoretical Perspective}

This study involves the assessment of the implementation of a multifaceted patient care model designed to improve quality of care and, consequently, chronic disease outcomes at safety net settings affected by numerous challenges, requirements, and complex patient populations. In understanding the implementation of this model and the influence that multiple factors at $\mathrm{CHCs}$ have on this process, this study places emphasis on three theoretical frameworks: The Expanded Chronic Care Model (ECCM), the Consolidated Framework for Implementation Research (CFIR), and the PCMH model itself. These three models provide valuable insights into the approaches to chronic care in 
these organizations, factors that affect implementation processes, and the principles of the PCMH model, respectively. Whereas the data collection phase of this research followed an inductive approach, it was important to align data collection guidelines with these theoretical frameworks to provide the researcher with the necessary direction on the field, considering the numerous dynamics that take place within complex organizations. In addition, research findings were interpreted in the light of these theoretical frameworks.

\section{Expanded Chronic Care Model}

Following the focus of this study, it is important consider the implementation of the PCMH model from the perspective of its contributions to chronic disease management and the improvement of health outcomes in patients with chronic disease. This is why this study uses the ECCM to analyze and interpret research findings. The ECCM is an "expanded" version of the Chronic Care Model (CCM), which is fundamental for this research because, as further discussed in Chapter 1, it set the standards for chronic disease management within these settings and led to the development of the PCMH (Setodji et al., 2017). The ECCM adds a population-based health promotion component as a result of the lessons learned through the implementation of the CCM at HRSA-funded community health centers as part of the Improving Chronic Illness Care collaborative (Glasgow \& Stange, 2014).

According to Barr et al. (2003), the integration of population-based health promotion into chronic disease prevention and management under the ECCM enhances efforts to further reduce the burden of chronic disease and supports communities in their efforts to be healthy. In addition. the model highlights the importance of considering the place of health care systems within a larger community environment and policy/cultural 
context, placing emphasis on the linkages between the health care setting and community resources (Glasgow \& Stange, 2014).

\section{Consolidated Framework for Implementation Research}

This study also used the Consolidated Framework for Implementation Research (CFIR) to ensure the focus on the contextual factors involved in the process of implementation of the PCMH and facilitate the analysis and interpretation of findings. The CFIR is key to understanding the relationship between context and implementation processes across a range of settings (Damschroder et al., 2013; Liang et al., 2016). This framework is especially useful in identifying and understanding barriers and facilitators influencing implementation of evidence-based practices in preventive and primary care (Liang et al., 2016). According to the CFIR, an intervention itself presents a series of characteristics that could either challenge or facilitate the implementation in multiple ways (Damschroder et al., 2013). However, the organization's external context and its inner setting play vital roles in the adoption or implementation of the project or intervention. Crucial inner setting factors include the organization's culture, the nature and quality of networks among organizational agents, the level of commitment to changes, and the availability of resources (Damschroder et al., 2013). Individual-level characteristics such as knowledge and attitudes of organizational agents can also influence the implementation process. Given its focus, this research placed emphasis on CFIR's constructs related to "inner setting", "characteristics of individuals", and “process". 


\section{Patient-Centered Medical Home Model}

The third and final theoretical framework guiding this research is the PCMH model. Obtaining PCMH recognition is the result of a formal process established by several national quality accrediting agencies. The standards or principles established by each of these agencies and other noteworthy quality organizations have been crucial to the development of guidelines for the adoption of the PCMH. Although the terms used by these organization may vary, most place emphasis on a set of common principles: 1) team-based care and practice organization activities; 2) need-based, evidence-based care; 3) community partnerships; 4) culturally and linguistically appropriate services; 5) patient-centered access and continuity; 6) care management and self-care support; 7) care coordination and continuity of care; 8) quality improvement; 9) recognition of patients' rights, responsibilities, and empowerment; 10) patient and family participation; and 11) relationship between the patient and the health care team (NCQA, 2017; AAAHC, 2013). The model's standards and principles are thoroughly discussed in Chapter 1.

\section{Significance of the Study}

The nearly 1,400 HRSA-funded CHCs in the US and its territories serve close to 27 million patients (HRSA, 2018a). Over 90\% are low-income and more than $60 \%$ are racial and/or ethnic minorities (HRSA, 2018a). These patients are affected by complex health needs, including multiple chronic conditions. Incidence of chronic conditions such as diabetes and hypertension is disproportionate among CHC patients (Taylor, 2004). Overall, $15 \%$ of patients served by $\mathrm{CHCs}$ have diabetes and $27 \%$ have hypertension. In $2017,33 \%$ of CHC patients with diabetes had A1c levels greater than $9 \%$ and $37 \%$ of patients with hypertension were uncontrolled (HRSA, 2018a). 
Even when many CHCs have had significant success improving the health outcomes of their populations, others have struggled. CHCs confront a myriad of challenges, including insufficient resources to offer comprehensive preventive and primary care to all community residents while investing to improve quality and efficiency; high workload; and high staff turnover (Shin et al., 2009; Quinn et al., 2013). Recruitment and retention of qualified health professionals willing to practice in medically underserved communities is another significant hurdle faced by $\mathrm{CHCs}$ (Rosenbaum et al., 2010). In addition, high proportions of those receiving care at CHCs have comorbidities, challenging social needs, and limited English proficiency or health literacy (Timbie et al., 2017). These competing priorities pose significant barriers to providing accessible, safe, affordable, and quality care (Smith et al., 2017).

Supporting the successful implementation of the PCMH at CHCs could help advance efforts to promote health, enhance quality of life, and reduce the number of lives lost prematurely among those with chronic disease. This study is expected to enhance the impact of CHCs on the quality of life of millions of disadvantaged individuals affected by chronic diseases nationwide. CHCs are an essential component of the US health system and play a key role in helping accomplish the Nation's overarching health goals: 1) attaining longer lives free of preventable disease, disability, injury, and premature death; 2) achieving health equity, eliminating disparities, and improving health;3) creating social and physical environments that promote health; and 4) promoting quality of life, healthy development, and healthy behaviors (Department of Health and Human Services, 2014). 


\section{Overview of the Dissertation}

The five chapters of this dissertation attempt to provide a thorough understanding of the context within CHCs operate to accomplish their mission and how this context shapes their capacity to implement the PCMH model and improve chronic disease outcomes. Chapter 1 contains a review of relevant literature on the implementation of the PCMH model at $\mathrm{CHCs}$ and key findings from previous related studies. It also discusses existing fundamental research gaps and the importance of conducting this study to address those gaps. Chapter 2 is a thorough discussion of the research methods and data collection procedures designed and implemented as part of this study, and the approach established to develop and analyze the two cases presented in chapters 3 and 4, respectively. These two cases are later cross-analyzed in Chapter 5, which includes the discussion of findings, recommendations, and conclusions, along with a proposed organizational framework for PCMH implementation at CHCs, based on findings and interpretations. 


\section{CHAPTER I}

\section{SIGNIFICANCE AND LITERATURE REVIEW}

As explained in the Introduction, successful implementation of the PatientCentered Medical Home (PCMH) model could provide HRSA-funded Community Health Centers (CHCs) an opportunity to enhance their performance, including their efforts to improve health outcomes for disadvantaged populations living with chronic diseases. CHCs play a key role in helping accomplish the nation's overarching health goals by providing care and support to over 27 million people across the US and its territories, 92\% of whom are low-income (HRSA, 2018a). These individuals are affected by multiple complex social and health needs, including co-occurring chronic diseases.

There is a need to understand why health centers with PCMH recognition have not been able to equally achieve anticipated chronic disease outcomes, why PCMH has only seemed to work effectively at certain $\mathrm{CHCs}$, and what differentiates successful models at CHCs. Placing emphasis on this need, this study aimed to explore and characterize organizational factors that distinguish or affect $\mathrm{PCMH}$ implementation at two CHCs with PCMH status recognized by HRSA as high-quality leaders with variations in context. This first chapter examines relevant literature on the implementation of the $\mathrm{PCMH}$ as a model designed to improve chronic disease management performance, particularly at safety net settings such as HRSA-funded CHCs. It presents key findings regarding the effectiveness of the model on chronic disease outcomes and existing challenges in implementing and assessing its impact, including the prevailing variability in PCMH implementation and outcomes. 


\section{The Impact of Chronic Diseases in Communities Served by CHCs}

Even when several chronic diseases are highly preventable, 150 million people nationwide live with at least one chronic disease and close to 100 million have more than one (Buttorff, C., Ruder, T., \& Bauman, M., 2017). Furthermore, nearly 30 million live with five chronic conditions or more (Buttorff et al., 2017) As stated previously, population groups served by CHCs across the US and its territories are largely impacted by chronic diseases. In 2017, among CHC patients nationwide, 15\% (nearly 2.3 million) had been diagnosed with diabetes, $27 \%$ (over 4.2 million) had hypertension, and close to 6\% (almost 1.3 million) had asthma (HRSA, 2018a). Some groups are disproportionately affected by chronic diseases, including ethnic minorities, who are nearly twice as likely as whites to have a chronic condition (Price et al., 2013). As discussed earlier, the majority of those served by $\mathrm{CHCs}$ belong to one or more ethnic/racial minority groups. In 2017, 9.4 million people served by CHCs nationwide (36\%) were Hispanic/Latino and over 5.2 million (22\%) were Black/African American (HRSA, 2018a).

The social characteristics of individuals served by CHCs also make them more vulnerable to being affected by chronic diseases. Due to the nature and mission of CHCs, the majority of their patients are also low-income, uninsured, and underserved. CHCs serve 1 in every 3 low-income uninsured individuals in the US and 1 in every 6 Medicaid beneficiaries (NACHC, 2017). In 2017, nearly 92\% of the population served lived below $200 \%$ of the poverty level and $69 \%$ lived at or below $100 \%$ of the poverty level (HRSA, 2018a). Half were Medicaid beneficiaries and 23\% were uninsured.

These population groups are at higher risk of having one or more chronic diseases. Research evidence shows that adults living below the poverty threshold are at 
greater risk for conditions such as cancer, depression, diabetes, behavioral health disorders, asthma, and stroke (Smith et al., 2017). In addition, those with lower incomes, less education, and uninsured are more likely to be affected by conditions such as cardiovascular disease and diabetes (Roger and Zhang, 2017; Towne et al., 2017; (Brown et al., 2011).

\section{Chronic Disease Management: A Fundamental Strategy for CHCs}

Achieving significant improvements in health outcomes among patients with chronic disease is crucial to improving their quality of lives and minimizing the risk of complications. Poorly managed chronic diseases can lead to major complications. For instance, annually, there are over 100,000 hospitalizations for a lower-extremity amputation due to diabetes and more than 160,000 for diabetic ketoacidosis (CDC, 2017). Furthermore, over 50,000 people with diabetes develop end-stage renal disease (CDC, 2017). Uncontrolled high blood pressure can increase the risk of heart attacks, stroke, heart failure, kidney disease or failure, vision loss, angina, and peripheral artery disease (American Heart Association, 2016).

Unfortunately, managing diabetes and other chronic conditions can be highly challenging for those affected. Individuals with chronic diseases deal with the complexity of their conditions on a daily basis, managing difficult lifestyle regimens and coping with the co-occurring psychological consequences (Schulman-Green et al., 2012). Common challenges for patients with chronic illnesses include recognizing symptoms and taking appropriate actions, using medications effectively, managing complex regimens, coping with the psychological consequences, and interacting with the healthcare system (Schulman-Green et al., 2012). The IOM (2012) has been emphatic 
about the need for funders, health systems, policy makers, and public health programs and agencies to come up with effective measures to enhance the quality of life of those living with chronic illnesses.

Multiple integrated and coordinated chronic disease management approaches have emerged in the past 20 years. However, many health systems continue to employ a reactive, episodic approach to care that responds to illnesses with sudden onset and limited duration (Nuño, Coleman, Bengoa, \& Sauto, 2012). CHCs are at the forefront of efforts to control hypertension and diabetes, especially as, among the low-income population, the likelihood of uncontrolled glucose levels and high blood pressure remains a concern. For years, CHCs have placed emphasis on integrated chronic disease management strategies such as the Health Disparities Collaboratives (HDC), based on the Model for Improvement and the Chronic Care Model (CCM), and, more recently, the PCMH model.

Edward H. Wagner, from the MacColl Institute for Healthcare Innovation, developed the CCM in 1998, in response to the high rates of chronic disease in the US. Wagner and colleagues wanted to address the existing need for a comprehensive approach by transforming health care into a proactive system focused on keeping individuals healthy, rather than just responding to acute episodes (Improving Chronic Illness Care, 2006). According to Wagner et al. (2001), effective management of chronic disease requires addressing the multiple challenges and barriers confronted by the patient and his or her family: dealing with symptoms, disability, emotional distress, complex medication regimens, lifestyle changes, social demands, barriers to access to care, and obtaining comprehensive care. 
According to Zwar et al., (2006), the overall aim of the CCM is to develop wellinformed patients and a health system that is ready for them. Based on the model, achieving the desired improvements requires actions that ensure patient-centered, timely, efficient, coordinated, evidence-based, and safe interactions between an informed, empowered patient and family and a proactive health care team (Improving Chronic Illness Care, 2014). For these interactions to take place effectively, six essential elements are required: community resources and policies, the health care organization, selfmanagement support, delivery system design, decision support, and clinical information systems (Figure 1). The health system is expected to incorporate information systems, self-management interventions, and decision support, as well as promote interactions between the health organization and the community (Improving Chronic Illness Care, 2014). All processes and relationships take place within a universe of three "overlapping galaxies": the entire community system, including its resources and policies, the health care system, and the provider organization (Bodenheimer, Wagner, \& Grumbach, 2002).

According to Wagner et al. (2001), chronic disease sufferers must build confidence and skills to manage the illness through access to community resources, adequate support, optimal care, and ongoing follow-up. Patients who are active, informed, and empowered cope better with the challenges of living and treating chronic illness (Wagner et al., 2001). The health system, thus, must design and implement strategies to ensure patients participate actively in their care and adopt effective selfmanagement practices. Glasgow et al. (2002) single out "self-management support" as a central feature of the model. Through self-management support, the health system is expected to help build the skills necessary for the patient's active engagement in chronic 
disease self-care practices (Bodenheimer et al., 2002; Green et al., 2012). This requires a "whole systems" perspective that acknowledges the central role of the patient (Kennedy, Rogers, \& Bower, 2007).

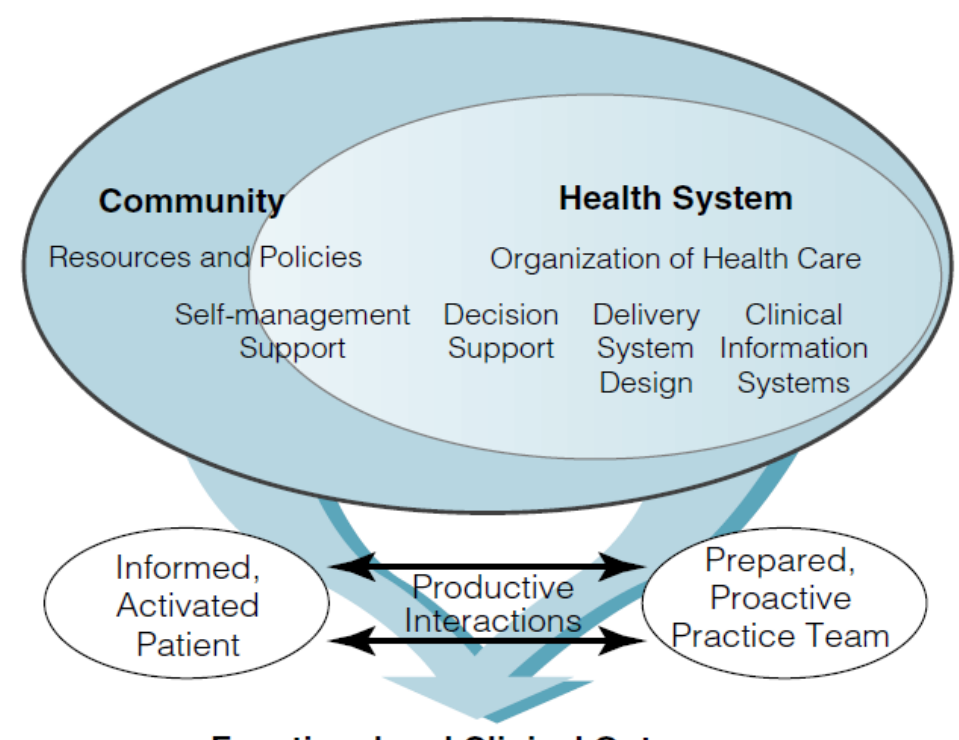

Functional and Clinical Outcomes

Figure 1. The Chronic Care Model. Reprinted from Wagner EH. Chronic disease management: what will it take to improve care for chronic illness? Effective Clinical Practice, 1998(1), 2-4. Reproduced with permission of American College of Physicians in the format Thesis/Dissertation via Copyright Clearance Center.

From 1998 to 2008, HRSA made the CCM a priority for CHCs through the implementation of the HDC, which prioritized continuous improvement of the health delivery system, patient self-management, and the use of information systems for decision making (Landon et al., 2007; Calvo, Calvo \& Bezold, 2008; Chin, 2010). Through team-based learning sessions, groups of $\mathrm{CHC}$ leaders would learn and share best practices on target chronic disease management areas (Chin, 2010). In its beginnings, the CHCs' Collaboratives focused on improving diabetes outcomes (Taylor, 2004). Over the 10-year period, CHCs also implemented HDC interventions targeting asthma, 
cardiovascular disease, depression, cancer, and HIV/AIDS. Since 2011, HRSA and the CHCs have placed emphasis on the PCMH as an organizational, integrated approach to promote the improvement of chronic disease outcomes.

\section{PCMH: An Organizational, Integrated Approach to Chronic Disease Management}

For nearly a decade, multiple health organizations in the US have turned their attention to the PCMH, a comprehensive approach to chronic disease management built on the principles of the CCM (Setodji et al., 2017). This model is considered to have the potential to improve health outcomes among those with chronic disease, improve quality of care, enhance patient experience, and reduce health care costs (Martsolf et al., 2012; Setodji et al., 2017). The PCMH model promotes the delivery of patient-centered, comprehensive, coordinated, accessible, and evidence-based care in a culturally competent manner, placing emphasis on the whole person, his/her family, and his/her overall health and well-being (Beacham et al., 2012; Platonova et al., 2016).

Several researchers consider the PCMH model particularly effective in managing populations with complex chronic care needs and comorbidities, such as those served by CHCs (Lieberthal et al., 2017; Rivo et al., 2016; Dobbins et al., 2018). According to Rivo et al. (2016), PCMH-related aspects such as comprehensive care, coordinated care, and patient engagement are key to addressing these conditions, as well as managing patient populations. In addition, the model's emphasis on increased accessibility and cultural competence make it highly beneficial for vulnerable and underserved populations (Platonova et al., 2016).

Some see the potential for a wide-scale shift in the US health care system with the spread of the PCMH model (Miller \& Baumgartner, 2016). More and more health 
organizations working under the PCMH model are moving from an individual care approach to a population health perspective. They are also transitioning from an exclusive focus on health care delivery to working actively on advocacy and community engagement (Miller \& Baumgartner, 2016). These organizations are also placing emphasis on partnerships with local and state health partners, multiple sectors, and communities, an approach that has characterized CHCs for more than five decades.

\section{PCMH Principles and Standards}

Obtaining PCMH recognition is the result of a formal process established by several quality accrediting agencies in the country. The standards or principles established by each of these agencies, as well as other noteworthy quality organizations, have been crucial in the development of guidelines for the adoption of the PCMH. Due to the significance of these standards in defining PCMH efforts and obtaining PCMH status, this study uses them as a means to assess PCMH implementation among participating CHCs. As further discussed in Chapter 2, PCMH standards and principles were an important reference in the development of data collection instruments. They were also used as a framework for the analysis and interpretation phases of the study.

Currently, most CHCs obtain PCMH recognition from the National Committee for Quality Assurance (NCQA). Organizations obtain a level 1, 2, or 3 recognition, based on their scores. NCQA standards and activities have continued to evolve, with its most recent redesign published in September 2017 (Lieberthal et al., 2017). While existing research on the PCMH is based on previous editions issued by the NCQA, the standards follow NCQA's basic PCMH approach: access to care, team-based care, population health management, patient care planning and management, care tracking and 
coordination, and performance measurement and improvement (Miller-Day et al., 2017). The six 2017 NCQA PCMH standards focus on: 1) team-based care and practice organization activities; 2) knowing and managing patients; 3) patient-centered access and continuity; 4) care management and support; 5) care coordination and care transitions; and 6) performance measurement and quality improvement (NCQA, 2017).

Under the "Team-Based Care and Practice Organization" concept, the health organization is expected to provide continuity of care, communicate roles and responsibilities of the medical home to patients/families/caregivers, and organize and train staff to provide effective team-based care (NCQA, 2017). "Knowing and Managing Your Patients" requires the health organization to use patient and community data to deliver evidence-based care that supports population needs and provide culturally and linguistically appropriate services. In addition to responding to needs internally, the organization must identify and establish connections to community resources to collaborate and direct patients to needed support (NCQA, 2017). The needs and preferences of the population must be considered when planning services and establishing standards for the provision of enhanced care on a $24 / 7$ basis, which is the basic requirement under the "Patient-Centered Access and Continuity" concept (NCQA, 2017).

The "Care Management and Support" concept involves the systematic tracking of tests, referrals and care transitions to achieve high quality care coordination, lower costs, improve patient safety and ensure effective communication with community providers (NCQA, 2017). The organization must demonstrate collaboration with patients/families/ caregivers to develop a care plan that addresses barriers and incorporates lifestyle goals. 
"Care Coordination and Care Transitions" focuses on the coordination of care across the multiple internal and external entities involved, from the health care team to community organizations. Finally, under the "Performance Measurement and Quality Improvement" concept, the health organization is expected to establish a culture of data-driven performance improvement on clinical quality, efficiency and patient experience, as well as engage staff and patients/families/ caregivers in quality improvement activities (NCQA, 2017).

Another popular PCMH recognition body among $\mathrm{CHCs}$ is the Accreditation Association for Ambulatory Health Care (AAAHC). This organization has particularly designed a process for HRSA-funded CHCs. Thus, the number of CHCs seeking AAAHC medical home recognition is recently increasing. As stated in the AAAHC's Medical Home On-Site Certification Handbook (AAAHC, 2013), the agency’s Medical Home standards include the following: 1) recognition of patients' rights and responsibilities; 2) effective governing and administrative infrastructure to support highquality patient-centered care; 3 ) relationship between the patient and the medical home team; 4) timely services that meet patients' needs; 5) patient empowerment and support to facilitate their responsibility for their care; 6) continuity of care; 7) maintaining comprehensive records and an efficient health information system; and 8) having an integrated, patient-centered quality improvement program.

After reviewing the characteristics of a PCMH, Wagner and colleagues (2012) identified a group of change concepts needed to be considered a fully developed PCMH, in alignment with the elements of the CCM. In order to achieve full PCMH transformation, according to Wagner et al. (2012), a health organization needs: 1) 
engaged leadership; 2) a quality improvement strategy; 3) empanelment; 4) continuous and team-based healing relationships; 5) organized, evidence-based care; 6) patientcentered interactions; 7) enhanced access; and 8) care coordination. Wagner et al. (2012) stress that the organizational culture needs to foster ongoing performance assessment and the identification of opportunities for improvement with the active involvement of patients and community members. Moreover, the health organization must foster patientcentered interactions through active patient involvement in decision-making, care, and self-management, while monitoring and respecting patient's needs, preferences, and values (Wagner et al., 2012).

Although Wagner's medical home "change concepts" are closely related to NCQA's PCMH recognition criteria, Wagner et al. (2012) state that NCQA's criteria place more emphasis on the availability of electronic data and information systems throughout the care cycle (Wagner et al., 2012). Wagner et al.'s (2012) PCMH change concepts are intended to guide the development and measurement of specific practice changes. A key distinctive of these change concepts is that they foster awareness of the unique needs, capability, and culture of the organization. They point out, nonetheless, that these change concepts are not specific enough to support PCMH implementation on their own; they can be taken as general guidelines or goals, but not the methods to reach the goals (Wagner et al., 2012). As discussed later in this chapter, this is a key issue highlighted by multiple PCMH researchers. 


\section{Effectiveness of the Implementation of the PCMH Model on Chronic Disease Outcomes}

Several studies, including randomized clinical trials, observational studies, and meta-analyses, have been conducted to assess the effectiveness of the PCMH model, especially with regards to chronic disease outcomes and quality improvement. Some have found that the implementation of PCMH components, independently and in combination, can improve health outcomes among people with chronic diseases and processes of care to support chronic disease management. Other studies have looked into the implementation of the model, focusing specifically on PCMH status recognition, rather than examining specific standards. Whereas some have found that PCMH recognition status and some PCMH elements are associated with improved processes of care, certain health outcomes, and patient experience with care, existing evidence on PCMH effectiveness to date seems to be inconsistent. Also, as emphasized in earlier in the Introduction as part of the statement of the research problem, researchers studying the PCMH model repeatedly point out the substantial variability in the way the model is implemented.

Morgan et al. (2014) conducted a systematic review to examine specific individual practice improvement strategies under the seven PCMH principles that drive improvements in glycemic control among people with diabetes. The study revealed two main PCMH principles that seemed to contribute to HbA1c improvements: 1) physiciandirected care with nursing or pharmacist care management support and 2) whole-person orientation with lifestyle modification support, also known as self-management support (Morgan et al., 2014). The latter included interventions using behavior theory to enhance 
patients' self-efficacy, goal setting, nutrition, physical activity, and psychological wellness. According to Morgan et al., (2014), both of these principles place emphasis on a team-based approach to care and the involvement of the patient as part of the health care team. It was difficult, however, to assess the effectiveness of other PCMH principles due to the high variability in how they were implemented (Morgan et al., 2014).

James et al. (2017) evaluated the effectiveness PCMH status on HbA1c outcomes for patients with type II diabetes from six clinics in rural, low-income communities in the Mississippi Delta. The researchers assessed HbA1c changes from 2007 to 2012 in diabetic patients from PCMH medical practices compared to patients from non-PCMH practices, using logistic regression analyses over time by cohort, controlling for age, race, and cohort-level measures of the first visit (James et al., 2017). At the end of the fiveyear period, the percentage of PCMH patients with type II diabetes with HbAlc equal or greater than 6.5 decreased from $87 \%$ to $70 \%$. Among non-PCMH patients, the proportion increased from $70 \%$ to $77 \%$. PCMH status was found to be significantly associated with observed improvements in glycemic control (James et al., 2017). While the study focused on the effectiveness of PCMH status as the main independent variable (recognized PCMH vs. non-PCMH), rather than the assessment of specific PCMH elements or interventions, James et al. (2017) attributed the observed improvement to changes in the level of patient involvement and adherence to follow-up appointments.

Another study attempted to determine the impact of the model by using an index that measured the level of PCMH implementation (Setodji et al., 2017). Setodji et al. (2017) examined associations between the level of PCMH and CCM implementation and patient experiences with care at 14 service delivery sites from a federally qualified $\mathrm{CHC}$ 
in California. The Medical Home Index, a self-rating tool used to facilitate PCMH transformation, was applied to evaluate each practice, placing emphasis on the chronic disease management domain to evaluate the value of the CCM within the PCMH model. Multivariate analyses showed that sites with high level of CCM implementation had more positive patient experiences regarding aspects such as provider communication and overall rating of the primary care provider, after controlling for the number of providers (Setodji et al., 2017). The researchers stressed the importance of specifically identifying PCMH elements associated with improved patient experience, as well as barriers to successful implementation.

Davy et al. (2015) examined 77 peer-reviewed research papers on the effect of the implementation of the elements of the CCM, the basis of the PCMH, in primary care settings in different countries, including the US. The systematic review included randomized clinical trials (RCTs), non-RCTs, observational cohort studies, crosssectional studies, case studies, and case series. Twenty-two of the 31 case studies or case series found an association between certain elements of the CCM and disease outcomes, including self-management support, delivery system design, decision support, clinical information systems, and enhanced case management support (Davy et al., 2015). Also, four of the six observational retrospective cohort studies reviewed found improvements in chronic disease outcomes and three of the 11 cross-sectional studies found associations between implementation of CCM elements and health outcomes (Davy et al., 2015).

Findings from the RCTs reviewed by Davy et al. (2015) were, however, inconsistent. Three of the 13 RCTs found significant changes in health outcomes from baseline for the intervention groups as a result of the implementation of several different 
CCM elements. Yet, between-group differences were non-significant and six other studies reported no intervention effect for any health outcome. Moreover, according to Davy et al. (2015), it was not possible to identify any optimal combination of CCM elements that would lead to improvements in health outcomes due to the considerable variability in the way the CCM elements were implemented, as well as in the combination of elements across different primary care settings.

These findings are in tune with the experience of PCMH researchers. For instance, a systematic review of RCTs and observational studies with comparison group conducted by Jackson et al. (2013) to evaluate the evidence on the effect of PCMH interventions on patient, staff, and economic outcomes found small to moderate positive effect on aspects such as patient experience and preventive care processes. Nevertheless, the researchers could not find sufficient evidence to determine effects of PCMH implementation on health outcomes. Jackson et al. (2013) noted that the studies reviewed varied greatly in the number and types of approaches used to implement PCMH core components.

Shippee, Finch, and Wholey (2017) determined that, while some aspects or degrees of implementation of the PCMH are associated with improved quality, others show limited benefits. After assessing quality of care provided and outcomes for five chronic diseases in a group of health centers with and without Minnesota's state-based medical come certification, Shippee et al. (2017) concluded that medical home certification was associated with higher adjusted rates of optimal care for all conditionspecific measures, including glycemic control, hypertension control, and asthma control, among others, except for 6-month remission of depression. Nevertheless, they noted that, 
whereas being a medical home patient was generally associated with better quality, there was large variability in performance regarding processes of care across participating health centers (Shippee et al., 2017).

In response to the observed inconsistency in findings regarding the effect of the PCMH model on chronic disease outcomes, particularly diabetes and hypertension control, Dobbins et al. (2018) conducted a cross-sectional, population-based study to determine whether PCMH recognition status was associated with diabetes control rates among CHCs. Whereas they found significant association between PCMH status and greater diabetes control rates, they stressed the need to determine which elements actually contribute to positive disease outcomes and are necessary and sufficient to achieve the desired outcomes (Dobbins et al., 2018). Others, such as Shippee et al. (2017), suggest the consideration of alternative medical home frameworks that are better suited or adaptable to implementation settings.

\section{Challenges in Studying the Impact of the PCMH model}

Jackson et al. (2013), Morgan et al. (2014), Davy et al. (2015), Shippee et al. (2017), in addition to other researchers such as Quinn et al. (2013) and Hoff (2010), have concluded that the main challenge in studying the impact of the PCMH on health care practice and health outcomes is the high level of variability in the implementation of the model and functional interventions carried out to comply with each PCMH component. Jackson et al. (2013) further state that, given the large variability in the way the model is implemented at different settings, not many have been able to assess the effect of the overall model. Variations in performance and results across health centers, according to Shippee et al. (2017), reflect differences in interventions, scope of implementation, or 
populations under study, since the PCMH does not follow a homogeneous design, but an adaptive approach. Therefore, health centers address PCMH principles or standards differently (Shippee et al., 2017).

While the PCMH model is constituted by a series of principles identified as best practices, once recognition is obtained, not every health organization implements the model as a whole. A study conducted by Ottmar et al., (2015) found that some health organizations struggle to put some of elements into practice once adopted. This could have an impact on the implementation of the whole model, as certain elements of the model, such as population health management and team-based approaches to care, determine the availability or well-functioning of other PCMH elements (Ottmar et al., 2015). Dobbins et al. (2018) point out that, while the PCMH recognition process by accrediting agencies is currently the officially recognized method to validate PCMH model implementation, there is a distinction between PCMH recognition and actual implementation. Therefore, $\mathrm{PCMH}$ recognition does not necessarily mean that an organization is actually using or implementing all the model's principles (Dobbins et al., 2018). This is worth considering in understanding why PCMH outcomes have not been equally achieved among CHCs.

Timbie et al. (2017a) conducted a cross-sectional analysis to assess the relationship between PCMH capabilities and processes of care and health outcomes among Medicare beneficiaries in a sample of $804 \mathrm{CHCs}$ seeking NCQA recognition. The researchers measured PCMH capabilities reported by CHCs to NCQA as part of their application (Timbie et al., 2017a). Even though they found a positive association between PCMH capabilities and quality of care, they observed large variation in the adoption of 
PCMH components. According to Timbie et al. (2017a), some organizations had lower capabilities to adopt elements such as care coordination and quality improvement, while others, including more advanced $\mathrm{CHCs}$, showed limited ability to implement populationbased strategies and self-management support efforts. It is worth noting that Timbie et al. (2017a) found limited evidence of larger effects on patient outcomes associated with increasing levels of PCMH capabilities. They point out that PCMH capabilities reported to NCQA were self-reported and, thus, may have been under or overstated.

Lieberthal et al. (2017) carried out a mixed methods study to explore the PCMH transformation practices employed by a group of 11 small-to-medium sized NCQArecognized PCMHs. The researchers quantitatively analyzed the changes made to transform to a PCMH and qualitatively explored why some features of the model were or not adopted. They found that all participating organizations had changed or implemented many of the standards during their transformation to a PCMH. Yet, there was high variation in the way they implemented the standards. Lieberthal et al. (2017) concluded that, since the NCQA does not require every activity to be implemented but rather a total number of points to achieve recognition, practices choose the activities they want to or can afford to implement.

After conducting a systematic review to identify research on the implementation of the PCMH model, the effectiveness of PCMH interventions, and the evolution of the model, Hoff, Weller, and DePuccio (2012) determined that PCMH is defined and operationalized in different ways. According to Hoff et al. (2012), unless everyday primary care settings implement multiple interventions that include a combination of the general principles of the model, they fall short of true medical home care. As shown by 
several researchers, including Hoff et al. (2012), Lieberthal et al. (2017), Timbie et al. (2017a), and Miller-Day et al. (2017), the combination of interventions can differ significantly from setting to setting. To date, there is no specific definition or standard on the strategies or interventions necessary to successfully achieve patient care and health outcomes (Hoff et al., 2012). The lack of specific guidelines and interventions to follow may further impact PCMH implementation in settings with considerable resource limitations such as CHCs and other safety net organizations.

According to DePuccio and Hoff (2014), PCMH care is a general umbrella term that includes a variety of different approaches, tools, and innovations to shape patient experience, practice efficiency, and disease management. The variation in how different primary care settings implement their version of the model has limited the possibility of gaining full understanding on how to take the model from simply a structure for the organization of care to an effective model with results at the patient care level (Hoff et al., 2012). Hoff (2010) sustains that the principles and concepts of the PCMH model have not been defined precisely and, thus, there is no blueprint for the implementation of the model. NCQA's PCMH tool to determine the readiness of clinical practices for PCMH implementation does not provide standards on how to implement each principle (Hoff et al., 2012; Hoff, 2010).

Multiple researchers have expressed concerns regarding whether or not different primary care practice settings, with different resources and constraints, should have similar PCMH interventions and outcome goals. Timbie et al. (2017) noted that some PCMH capabilities, such as population-based strategies and self-management support, may be highly complex for all community health centers, despite their level of PCMH 
capability, given their challenging contexts. Goldman et al. (2015), Quinn et al. (2013), and Hoff et al. (2012) have suggested that PCMH implementation and success differ according to organizational factors that have yet to be defined. Goldman et al. (2015) stress the need of understanding why the PCMH may only work in some contexts and how organizational factors are involved in achieving PCMH outcomes. According to them, the ongoing focus on clinical benchmarks to determine the model's effectiveness has left these important questions behind (Goldman et al., 2015).

\section{Importance of Organizational Context and Dynamics in the Success of the PCMH}

Due to the fact the PCMH manifests differently across settings and circumstances, understanding the model and making it work requires understanding the context in which it takes place (Agency for Healthcare Research and Quality, 2013). Several researchers, including Goldman et al. (2015), Quinn et al. (2013), and Hoff et al. (2012), have questioned whether different primary care settings, with different resources and constraints, should be expected to have similar PCMH interventions and outcome goals. According to Goldman et al. (2015), whereas the PCMH model aims at successful patient care outcomes, success may be affected by a series of undefined and variable organizational factors. The present study seeks to define, characterize, and provide direction in understanding these factors and how they shape the success of PCMH implementation.

Davy et al. (2015) highlight how some of the case studies they reviewed show the importance of developing interventions that are "contextually relevant". Moreover, they point out that the context where these interventions take place is particularly important when dealing with primary care settings serving disadvantaged populations in 
underserved areas such as CHCs (Davy et al., 2015). Determinant factors, according to these researchers, include organizational culture and team dynamics. For instance, they found that the use of Plan-Do-Study-Act (PDSA) cycles and learning collaboratives are associated with the implementation of contextually relevant interventions. Both PDSA and learning collaboratives engage health providers in development and implementation processes, encourage a sense of ownership, and foster the use of "reflective practice" strategies (Davy et al., 2015).

The Agency for Healthcare Research and Quality (AHRQ) (2013) has stated that, what works in one context does not work in another. Furthermore, the AHRQ (2013) indicates that several factors can be crucial to the implementation and potential impact of the PCMH. These factors include: national, State, local, and organizational policies; community norms and resources; health care system organization; payment and incentive systems; practice culture, history, and staffing; characteristics of patient populations and subgroups; historical factors and recent events; the culture and motivations surrounding monitoring and evaluation; and changes in these factors over time (AHRQ, 2013).

According to Ackroyd and Wexler (2014), effectively implementing the PCMH model requires solid leadership, active involvement of staff in both planning and implementation, and the incorporation of strategies that enhance morale and motivation. These conditions foster improved organizational processes, improved care, and, thus, improved health outcomes. Davy et al. (2015) also stress the key role of leadership, not only in the development and implementation of chronic care strategies, but also in fostering opportunities for collaboration, staff involvement, and sustainability of these 
interventions. They emphasize the need to identify facilitators and barriers to the implementation of chronic disease models.

Miller-Day et al. (2017) studied the experiences of four health centers transitioning to the PCMH model as part of a state-wide Chronic Care Initiative. Two of these organizations were classified as "high-improvement (HI)" and two as "lowimprovement (LI)", based on their chronic care performance during an 18-month period. One of the two low performing health care organizations was a small $\mathrm{CHC}$; the remaining three participating institutions included an internal medicine private practice, a large family medicine residency program within a larger health system, and a small physicianrun group practice collaborative. This study qualitatively described the experience of these organizations as they transitioned to the adoption and implementation of the PCMH model (Miller-Day et al., 2017). The research's main focus was to identify facilitators and barriers to $\mathrm{PCMH}$ adoption and transformation, placing emphasis on ways each PCMH standard was adopted by low and high performers.

According to Miller-Day et al. (2017), major facilitators to PCMH adoption and transition among the more successful performers included: engaged leadership, teambased approach, sense of ownership, staff buy-in, and the use of health information systems. The most important barrier identified among low performers was the disconnect between leadership and clinical staff and employees. Miller-Day et al. (2017) also noted that participating health organizations had different approaches to the implementation of the PCMH standards, as well as mixed interpretations of the patient-centeredness concept, even when they were all working within the framework set under the PCMH model (Miller-Day et al., 2017). 


\section{The PCMH within the Context of HRSA-Funded CHCs}

The literature documented in the previous section shows the importance of understanding how organizational context influences the success of PCMH implementation and the achievement of chronic disease management outcomes. While this should be a key issue for every health organization implementing this model, it seems crucial for HRSA-funded CHCs. As discussed in the Introduction, even when $\mathrm{CHCs}$ have historically provided comprehensive patient-centered care, $\mathrm{PCMH}$ implementation in these settings is challenged by multidimensional factors (Timbie et al., 2017b). Due to their nature and mission, CHCs serve low-income underserved populations, mostly racial/ethnic minorities, affected by multiple chronic diseases and a variety of socioeconomic factors that challenge access to care, adherence to regular care, and full engagement in disease management plans (Whelan, 2010; NACHC, 2017; HRSA, 2017; Perez et al., 2013). Organizationally, CHCs must confront numerous hurdles, including financial constraints, difficulties in recruiting necessary staff, high staff turnover, and high workloads (Shin et al., 2009; Rosenbaum et al., 2010; Quinn et al., 2013; Perez et al., 2013).

According to Anderson and Olayiwola (2012), the PCMH model requires substantial changes in care processes, organizational practices, and quality improvement efforts. It also requires availability of quality improvement experts, openness to change, strong teamwork skills, and significant organizational culture transformation.

Furthermore, Anderson and Olayiwola (2012) stress the need for high levels of adaptive reserve. According to Tu et al. (2015), adaptive reserve provides the necessary flexibility and resilience in times of change. 
The obstacles faced by CHCs may limit their capacity to adapt their organizational and work cultures to the demands of care transformation models such as the PCMH and, thus, their capacity to bring about the desired improvements in quality of care and disease outcomes (Perez et al., 2013). Perez et al. (2013), who conducted an exploratory study on patient-centered care models at six safety net organizations aiming to improve chronic outcomes in underserved populations, including one HRSA-funded $\mathrm{CHC}$, noted that these organizations find it harder to have the staff needed to become a PCMH and enhance quality improvement. Also, according to Perez et al. (2013), having transient populations and populations with unstable insurance coverage make it difficult to implement PCMH principles such as care continuity. Additionally, safety net organizations struggle with the implementation of team-based care due to high turnover and the difficulty in recruiting physicians and other health professionals. Research that supports PCMH implementation within the context of CHCs is still highly needed.

\section{Summary of Evidence and Research Gap}

There is vast evidence that $\mathrm{CHCs}$ ' comprehensive approaches to care have been effective in reducing complications in patients with chronic conditions (Taylor, 2004). Furthermore, the Institute of Medicine (IOM) and the General Accounting Office (GAO) consider $\mathrm{CHCs}$ as models for chronic disease screening, diagnosis, and management (Ravenswood Family Health Center, 2014). However, as documented in this chapter, over time, there has been wide variation in the quality of care provided and results

obtained across CHCs; while some exceed performance indicators, others perform poorly (Chin, 2010). 
HRSA promoted and encouraged initiatives such as the HDC and the PCMH as ways to support improvements in quality of care and chronic disease outcomes at CHCs. The PCMH was adopted as a promising approach in a time when quality and costreduction were seen as priorities for the nation's health system. Nevertheless, whereas multiple studies conducted at different primary care settings found associations between several PCMH-related interventions and improved chronic disease outcomes, evidence has been inconsistent (Davy et al., 2015; Shi et al., 2015; Morgan et al., 2014). In addition, it is uncertain which strategies bring about successful chronic disease management outcomes (Ackroyd \& Wexler, 2014; Goldman et al., 2015). The combination of interventions differs significantly from setting to setting as there is no definition or standard on the strategies or interventions necessary to successfully achieve patient care and health outcomes (Hoff, Weller, \& DePuccio, 2012). In fact, it has been documented that, once PCMH recognition is obtained, not every organization implements the PCMH model as a whole, particularly if the organization lacks the necessary resources and capabilities (Ottmar et al., 2015; Timbie et al., 2017a).

Some researchers highlight the lack of a blueprint for the implementation of the model as one key issue affecting PCMH implementation and, thus, its outcomes (Hoff et al., 2012; Hoff, 2010). Others express concerns regarding whether or not primary care practice settings with different resources and constraints should have similar PCMH interventions and outcome goals (Timbie et al., 2017). Mainly, there seems to be consensus among different PCMH researchers that PCMH implementation and its success differ according to organizational context and factors that have yet to be defined 
(Hoff et al., 2012; Quinn et al., 2013; Ackroyd \& Wexler, 2014; Goldman et al., 2015; Miller-Day et al., 2017).

Several researchers have explored the experiences, barriers, and facilitators during the transition to PCMH adoption at different medical practice settings. Perez et al. (2013), for instance, explored the views of experts at six safety net organizations aiming to improve chronic outcomes in underserved populations through patient-centered care models, such as the PCMH and the Accountable Care Organization (ACO), on facilitators and barriers to the transformation of the health care delivery system to inform the next phases of PCMH and ACO development. Miller-Day et al. (2017) studied variations in PMHC transformation and adoption strategies in high and low improvement medical practices, according to a set of quantitative chronic disease measures (Miller-Day et al., 2017). Both studies placed emphasis on factors that hinder or facilitate practice transformation in adopting PCMH standards as part of the process to become a PCMH.

There are still important questions regarding the dynamics behind an organization's capacity to succeed at implementing the PCMH model and producing improvements in chronic disease management practices and outcomes, especially among HRSA-funded CHCs. Since 2011, HRSA has awarded millions of dollars in federal funding to promote quality improvement, $\mathrm{PCMH}$ implementation, and improvements in chronic disease outcomes among CHCs. Yet, variations in $\mathrm{CHC}$ performance, $\mathrm{PCMH}$ implementation, and chronic disease outcomes across CHCs nationwide suggest the existence of factors beyond PCMH principles and HRSA support affecting CHC performance. As presented earlier, key health outcomes such as diabetes and hypertension control are met by only $37 \%$ and $15 \%$ of CHCs, respectively (NACHC, 
2017). In 2017, whereas over $75 \%$ of CHCs were certified as PCMHs, less than one-third had achieved HRSA recognition as quality leaders and less than 3\% as national quality leaders (HRSA, 2018b).

This research addresses the need to understand why anticipated PCMH outcomes with regards to chronic disease management have not been equally achieved and how organizational factors affect PCMH implementation at CHCs. As stated in the Introduction, the specific aim of this research is to explore and characterize organizational factors that distinguish or affect PCMH implementation at $\mathrm{CHCs}$, focusing on two CHCs with PCMH status recognized by HRSA as quality leaders. A qualitative collective case study was designed and implemented to support the accomplishment of this aim. Chapter 2 provides further details on the research strategy selected, study settings, data collection methods, and data analysis procedures.

\section{Summary of Challenges and Factors Involved in PCMH Implementation}

Table 1 summarizes the major factors and barriers related to PCMH implementation identified in the exiting literature, as discussed throughout this chapter.

\section{Table 1}

Major factors and barriers to PCMH implementation identified in PCMH literature Challenges in understanding the impact of the PCMH model on health care and health outcomes

a. PCMH recognition does not necessarily mean that an organization is using or implementing all the model's principles (Dobbins et al., 2018).

b. Researchers have noted high level of variability in the implementation of the model and functional interventions carried out to comply with each PCMH component 
(Jackson et al., 2013; Morgan et al., 2014; Davy et al., 2015; Shippee et al., 2017; Quinn et al., 2013; and Hoff, 2010).

c. Variations in performance, interventions, and scope of implementation may be a result of the design of the PCMH, since the model does not follow a homogeneous design, but an adaptive approach (Shippee et al., 2017).

d. Researchers have also found mixed interpretations of the patient-centeredness concept, even when they were all working within the framework set under the PCMH model (Miller-Day et al., 2017).

e. The combination of PCMH interventions can differ from setting to setting (Hoff et al., 2012; Lieberthal et al., 2017; Timbie et al., 2017a; Miller-Day et al., 2017).

\section{Barriers to the implementation of the PCMH model}

a. Some health organizations exhibit lower capabilities to put into practice several elements of the PCMH model once adopted, including care coordination and quality improvement, population-based strategies, and self-management support efforts (Ottmar et al., 2015; Timbie et al., 2017).

b. Since accrediting agencies do not require every activity to be implemented but rather achieving a number of points to achieve recognition, practices choose the activities they want to or can afford to implement (Lieberthal et al., 2017).

c. To date, there is no specific blueprint for the implementation of PCMH strategies or interventions necessary to successfully achieve patient care and health outcomes (Hoff et al., 2012; Hoff, 2010).

d. Lack of specific guidelines and interventions to follow may further impact PCMH implementation in settings with considerable resource limitations such as $\mathrm{CHCs}$ and other safety net organizations (Hoff et al., 2012; Lieberthal et al., 2017; Timbie et al., 2017a; Miller-Day et al., 2017).

e. Researchers have expressed concerns regarding whether or not different primary care settings, especially those serving disadvantaged populations affected with different constraints, should have similar PCMH interventions and goals (Goldman et al., 2015; Davy et al., 2015).

f. Numerous hurdles may limit CHCs capacity to adapt their organizational cultures to the demands of PCMH transformation and to comply with PCMH requirements, including: financial constraints, difficulties in recruiting necessary staff, high staff 
turnover, unstable patient populations, and high workloads (Shin et al., 2009; Rosenbaum et al., 2010; Quinn et al., 2013; Perez et al., 2013).

Potential factors involved in PCMH implementation at different health care settings

a. Several researchers have proposed a series of factors that support PCMH adoption and could drive PCMH success:

1) Organizational culture and team dynamics (Davy et al., 2015)

2) Engagement of health providers in development and implementation processes and reflective and learning strategies (Davy et al., 2015)

3) Active involvement of staff in both planning and implementation, and strategies that enhance morale and motivation (Ackroyd and Wexler, 2014)

4) Leadership engagement (Ackroyd and Wexler, 2014; Davy et al., 2015; Miller-Day et al. (2017)

5) Involvement of leadership in the development and implementation of chronic care strategies (Davy et al., 2015)

6) Opportunities for collaboration and staff involvement in the development and implementation of chronic care strategies (Davy et al., 2015)

7) Team-based approach, staff buy-in, and the use of health information systems (Miller-Day et al., 2017)

8) Availability of quality improvement experts, openness to change, teamwork skills, and organizational culture transformation (Olayiwola, 2012)

9) High levels of adaptive reserve, flexibility and resilience in times of change (Olayiwola, 2012; Tu et al., 2015) 


\section{CHAPTER II}

\section{METHODS, DATA COLLECTION, AND ANALYSIS PROCEDURES}

The literature review presented in Chapter 1 shows that one of the main challenges in implementing the PMCH model is the large variability of interventions used under each of the model's principles, not only leading to diverse results but making it hard to assess the model's effectiveness. Furthermore, PCMH implementation is affected by the absence of guidelines on specific potential interventions. Especially, there are few, if any, directions regarding the implementation of contextually relevant strategies. The ambiguity of the model has led researchers and evaluators to establish clinical benchmarks to determine its effectiveness, leaving behind relevant questions regarding

why anticipated outcomes have or not been achieved, how and why the PCMH may work in some contexts, and how organizational factors affect PCMH implementation (Goldman et al., 2015). These are key questions in expanding existing knowledge regarding PCMH effectiveness, particularly in HRSA-funded CHCs.

The qualitative collective case study presented in forthcoming chapters examines the organizational-level factors that distinguish and affect PCMH implementation and the achievement of improved chronic disease management at two participating CHCs with PCMH recognition and HRSA quality recognition. This chapter presents a thorough discussion of the research methods and data collection procedures used in this study. It also discusses the approach followed in developing and analyzing the two cases presented in chapters 3 and 4, respectively. The first section of the chapter describes the overall methods of the study, while the second section describes the data collection procedures. 
The third section provides details about the procedures followed to analyze data collected. The chapter ends with a discussion of strategies used to enhance the quality and rigor of the study and ethical considerations.

\section{Methods}

\section{Overall Research Strategy}

In conducting the above research, a qualitative design was found to be most appropriate to the objective of the study: to identify gaps in PCMH implementation and contributors to effective chronic disease management. As already noted in Chapter 1, the latter is a fundamental part of the work of HRSA-funded CHCs. More importantly, the data generating process in this type of design is dynamic, with the researcher actively becoming an observer at the $\mathrm{CHC}$. This approach was found to be essential to yielding the data needed to construct a contextualized understanding of the day to day activities and processes taking place at $\mathrm{CHCs}$ and how these may influence $\mathrm{PCMH}$ implementation (Goldman et al., 2015). Additionally, qualitative research provides an opportunity to explore the phenomenon of interest within its natural setting and understand how it develops within the conditions in which CHCs operate (Creswell, 2013; Denzin \& Lincoln, 2003). Hence, qualitative methods were used to collect data presented and analyzed in the following chapters, which allowed the researcher to examine the complexities of the PCMH model and the dynamics of the adaptive strategies devised at the settings where PCMHs are implemented, as suggested by Damschroder et al., 2013.

Creswell (2013) points to a series of key attributes of the qualitative research design. These fundamental characteristics highlight the importance of employing this design in approaching the aim and objectives guiding this study. In addition to facilitating 
the study of a phenomenon within a particular context or setting of interest, qualitative research was selected for its emergent, evolving design (Creswell, 2013). This was expected to allow the exploration of multiple factors and dimensions related to PCMH implementation and chronic disease management at participating $\mathrm{CHCs}$ as they emerged during site observations, in addition to drawing on different strategies and methods to obtain the information needed. Finally, qualitative research is also reflective and interpretive, engaging the researcher in an ongoing interaction with the data to produce lessons learned throughout the process (Creswell, 2013).

Another essential characteristic of the qualitative research design, according to Creswell (2013), is that it allows researchers to develop a complex picture of the phenomenon under study, which is fundamental within the complex context of PCMH implementation. Gathering and reporting multiple perspectives, using a variety of sources of data, provide the elements needed to identify the numerous factors involved in the phenomenon and develop a holistic description. Rather than looking for cause-effect relationships between factors, the main interest of qualitative methods lies on identifying the complex interactions among factors involved (Creswell, 2013). As supported in the following chapters, a qualitative design was needed to accomplish the detailed understanding of the PCMHs that will be presented, which involves an array of complex structural factors and human actors interacting within multifaceted settings.

\section{Case Study Approach}

Within the multiple qualitative approaches available to the investigator, a qualitative case study approach was used to examine the phenomenon under study within the setting of interest through multiple perspectives (Schadewaldt et al., 2014; Yin, 
2003). A "case" is considered a bounded, integrated system constituted by multiple actors, programs, processes (Stake, 1995; Creswell, 2013). According to Creswell (2013), this methodology provides access to an array of sources of information to develop an indepth understanding of complex issues in settings with well-defined boundaries that can be delimited or described within a series of parameters (Creswell, 2013). It is also ideal when the focus is on both the phenomenon and its context, such as the case of PCHM implementation at CHCs (Yin, 2003). The particular approach of this study can be considered "instrumental", as it was not only designed to produce a description of a setting or the process, but to generate a better understanding of the specific issue of concern (Stake, 1995; Creswell, 2013).

The case study provided the best strategy for studying two contextually-different HRSA-funded CHCs with PCMH status, recognized by HRSA as high-quality leaders. Moreover, it is expected that conducting multiple case studies will yield more substantial and comparable data and, correspondingly, more rigorous findings, not likely to emerge from a single case (Stake, 1995; Schadewaldt et al., 2013). Furthermore, according to Gray (2013), data from two case studies multiply observations important in illustrating the phenomenon of interest, instead of reaching conclusions based on one case, thus multiple cases strengthen the validity and reliability of the study.

\section{Setting and Unit of Study}

The unit of study in this research is the HRSA-funded CHCs (cases), which also provides the setting. The main emphasis is on the "health center" as a dynamic setting where multiple processes take place as part of the implementation of the PCMH. To document variations in the implementation, organizational context, and organizational 
culture of the PCMH, the cases selected for the study included one case from a group of HRSA-funded CHCs in South Florida and a second case from a group of CHCs in the US Commonwealth of Puerto Rico. Both organizations are part of a group of CHCs recognized by HRSA as "Health Center Quality Leaders" for having the best overall performance among all CHCs or exceeding national quality benchmarks, including Healthy People 2020 goals, for chronic disease management and preventive care. These performance indicators are annually assessed by HRSA, as CHCs are required to report a series of quality measures on an annual basis through the Uniform Data System (UDS).

Since 2014, HRSA-funded CHCs meeting or exceeding objectives for preventive care and chronic disease management measures annually receive Quality Improvement Awards (QIAs) to reward their achievements and support further improvement (HRSA, 2018b). CHCs exceeding national quality benchmarks, including Healthy People 2020 goals, for chronic disease management and perinatal/prenatal care are recognized as "National Quality Leaders". Those with the best overall performance among all CHCs are recognized as “Health Center Quality Leaders”. Based on the 2017 HRSA performance measures, one $\mathrm{CHC}$ in Florida was recognized as National Quality Leader and 18 were recognized as Health Center Quality Leaders, ten of which are located in South Florida (HRSA, 2018c). Among CHCs in Puerto Rico, three were recognized as National Quality Leaders and nine as Health Center Quality Leaders (HRSA, 2018c). In 2017, there were 47 HRSA-funded CHCs across the state of Florida, 17 of which serve communities throughout South Florida (HRSA, 2018b). Over $80 \%$ of CHCs in the state of Florida have PCMH recognition; nearly $77 \%$ of CHCs in South Florida are recognized PCMHs. Together, CHCs in Florida served nearly 1.5 million patients in 
2017, more than 390,000 in the South Florida region (HRSA, 2018b). The majority of patients served in South Florida were low-income individuals from a racial/ethnic minority group, half were Hispanic/Latino and 35\% Black/African American (HRSA, 2018b).

There were 20 HRSA-funded CHCs in Puerto Rico, $65 \%$ of which are recognized as PCMH. These organizations served close to 360,000 people in 2017,1 in every 10 individuals living in this US territory (HRSA, 2018c). Most patients served by CHCs in Puerto Rico live at or below $100 \%$ of the federal poverty level. It is worth noting that nearly all (99\%) of those served by HRSA-funded health centers in the Island are Hispanic, mainly Puerto Rican; less than 1\% are non-Hispanic white (HRSA, 2018c). These CHCs serve a culturally-homogeneous population with a level of sameness not seen at any other CHC in US mainland. CHCs in Puerto Rico and the communities they serve also confront unique conditions due to the financial crisis affecting the Island's population and basic systems, including health care. Additionally, health organizations in Puerto Rico are impacted by the existing disparities in Medicaid funding, compared to the states (Rios, 2017). The financial and infrastructure situation of these organizations worsened after the direct hit of the Island's worst hurricane in 100 years in September 2017.

\section{Sampling and Recruitment}

The study employed a purposeful and theoretical sampling approach. According to Creswell (2013), sites can be selected "purposefully", following certain criteria of interest, to inform the understanding of the phenomenon under study. As will be noted in forthcoming chapters, data collection took place at two HRSA-funded community health 
centers with PCMH recognition. Each $\mathrm{CHC}$ is a dynamic, complex setting where multiple processes take place as part of a patient-centered strategy to improve care and health outcomes. Moreover, in this study, information-rich and intense cases were purposefully selected to show different perspectives, issues, and factors involved in the phenomenon (Patton, 2002; Creswell, 2013).

Cases selected represented two theoretical samples, selected on the basis of the potential manifestation of a series of processes and constructs that are fundamental for the purpose of this study (Cohen \& Crabtree, 2006). Both participating organizations represented a desired level of performance and quality of care to increase opportunities of exploring successful models of PCMH implementation at CHCs. As mentioned previously, at the time of the study, these PCMHs were recognized by HRSA as highquality performers for meeting or exceeding a series of performance indicators for chronic disease management and preventive care.

The study's cases, presented in Chapters 3 and 4, were also purposefully selected to reach maximum variation in organizational context, since both achieved the desired performance and quality levels amid very different contexts and organizational cultures. Using the maximum variation sampling technique, where a criterion is used to select sites that are different on the particular criterion, allows the maximization of the differences at the beginning of the study to increase the likelihood that findings include multiple, different perspectives, circumstances, or practices (Patton, 2002; Creswell, 2013). The decision was made to allow for the documentation of unique variations as these were expected to emerge by conditions present at each site, but, moreover, it would provide an 
opportunity to explore the organizational adaptation of the PCMH implementation. This was essential in achieving the study's aim and research objectives.

The selection of two cases was expected to make it possible to achieve greater indepth exploration of the phenomena in question, especially through the triangulation of multiple sources. These sources were anticipated to yield rich data to allow for "thick" descriptions, as posited by Geertz (1973), of these centers and the processes through which they implement PCMH guidelines. In sum, this study was expected to comprehensively document and analyze the particularities of the context. The small sample selected would allow many opportunities to identify themes and conduct crosscase theme analysis (Creswell, 2013). According to Creswell (2013), qualitative case study research should focus on no more than four cases. Furthermore, experts indicate that every additional case over one dilutes the level of detail that can be provided by the researcher (Creswell, 2013).

Top quality performers in South Florida and Puerto Rico were identified from the list of quality awardees publicly available in HRSA's website. An open invitation was sent to five CHCs in South Florida, one CHC that had been recognized as National Quality Leaders at the time and four Health Center Quality Leaders with high performance in chronic disease management measures, as identified through the HRSA Health Center Data portal. Three CHCs in Puerto Rico recognized as National Quality Leaders were also approached. Three health centers in South Florida and one in Puerto Rico notified their interest in the study. Introductory meetings were held with these organizations to present details about the study protocol and address any concerns, especially with regards to confidentiality and protection of health information. In the end, 
one CHC in South Florida and one in Puerto Rico accepted to participate in the research. These organizations were provided with a brief case statement for them to present to other health center leaders, including board members, and an informational letter to be distributed among key informants participating in the study. The latter has been included in Appendix B.

\section{Data Collection Procedures}

Multiple data collection methods were used to obtain an in-depth understanding of each case: 1) non-participant direct observations, 2) document review; and 3) semistructured interviews. In collecting data from various sources and through several methods, this study intended to provide multiple measures of the same phenomenon, facilitating corroboration of the evidence gathered, inclusion of diverse perspectives, and greater understanding of the main issues of concern (Yin, 2003; Creswell, 2013).

\section{Non-Participant Direct Observations}

The data collection process began with non-participant field observations to capture organizational factors, processes, behaviors, experiences, and dynamics. Observation is a crucial method in developing case studies because it allows gaining indepth understanding of the phenomenon, adding an outsider perspective to internal perspectives and perceptions (Stake, 1995; Creswell, 2013). This data collection method contributes to the development of a multidimensional picture of the phenomenon, as it allows the researcher to submerge in the setting, language, culture, and overall experience, capturing the essence of what happened beyond formal activities (Creswell, 2013; Patton, 2002). Understanding the context was not only essential to obtaining a holistic perspective, but also central to the aim of this study (Patton, 2002). 
The purpose of conducting observations in the first phase of data collection was getting into the field without any preconceptions caused by health center staff perspectives. Employing this technique presented an opportunity to move beyond selective perceptions of key informants and provided access to first-hand information about the organizational context without being held captive by those selective perceptions (Patton, 2002).

While openness is part of the inductive nature of qualitative research, an observation protocol was used to organize observations and standardize the process at participating sites, taking into consideration the complexity of these settings and the phenomenon to be observed. The instrument provided guidelines throughout the process. Scales and/or checklists were designed under each category to facilitate observation and documentation. It also served as a recordkeeping tool. Following the essence of the qualitative methodology, reflection and introspection were part of the field research process (Patton, 2002). Thus, field notes were also used to document researcher reflections. Field, descriptive, and reflective notes regarding the experience and learnings were documented through this tool. All field notes were taken from a distance without any involvement in the dynamics. A separate protocol was used for each day of observation at each site. The observation protocol has been included in Appendix C.

PCMH principles and standards were used in the development of the observation protocol given their key role in PCMH recognition and implementation processes. Key PCMH implementation elements considered in designing the instrument included enhanced access, patient support/enabling services, after-hours, continuity of care, and care coordination dynamics. Elements of the Consolidated Framework for 
Implementation Research (CFIR), one of the theoretical frameworks guiding this research, were also incorporated to maintain focus on organizational factors. This framework focuses on elements such as organizational structure; leadership roles and behaviors; and collaboration and partnership activities. Even though these were not the only guidelines considered, they provided an important basis to maintain the necessary focus within the complex, multidimensional settings observed.

Field observations focused on aspects such as physical appearance and overall environment; accessibility; patient flow/work flow; facilities and resources available for patients; services provided; working hours and availability of extended hours; accessibility during after-hours; patient load throughout different times observed; patient waiting time throughout period of observation; patient characteristics (ages, cultural diversity, gender, among others); and cycle time (how patients moved from one process to the other throughout the visit). Patient, health care team, and health center staff behaviors and interactions, as well as respect for and management of cultural diversity, were also of high importance throughout the field observation process. Other observations placed emphasis on educational and general content disseminated through screens in waiting areas; announcements for patients; brochures and literature available for patients; and use of an electronic health record and availability of a patient portal.

\section{Document Review and Analysis}

The observation phase was followed by the review and analysis of texts found in documents related to the implementation of the PCMH model. According to Yin (2003), document review and analysis in case studies help corroborate evidence from other sources. Furthermore, Patton (2002) stresses the importance of organizational documents 
in providing insight to "behind-the-scenes" processes and the complex logistics of the organization's programs. Participating health centers provided organizational and public documents that served as evidence of the implementation of PCMH elements, particularly those related to chronic disease management policies and procedures. Documents submitted by the health center to complete the PCMH recognition process such as selfassessment tools, samples of practices implemented, minutes, and PCMH-related organizational policies were of particular interest.

A document review protocol, included as Appendix D, facilitated recording of observations, topics, and categories during the review of PMCH-related documents, paying attention to patient flow; patient/staff communications; use of technology; comprehensiveness of services provided; and a series of PCMH elements. These included: team-based care; care coordination (internal and external); patient-centered care; population management; collaboration between care team and patient/ family /caregiver; incorporation of patient preferences; self-management support; shared decision-making; community linkages; enhanced access to care; medical home responsibilities; and provision of patient support services. The CFIR was also used to design this instrument, with emphasis on organizational factors such as cultural competence; strategic partnerships; planning practices; leadership culture; patient participation; characteristics of the inner organizational setting (e.g., structure, networks, communications, culture, organizational dynamics, learning climate, quality improvement practices, leadership involvement, organizational resources); and staff characteristics (e.g., skills, roles, approach to care, teamwork). 
After gathering the documents and texts, the developed tool helped organize, guide, and standardize document review and documentation of findings, based on the aspects discussed above. Contents were also carefully reviewed and qualitatively analyzed to find significant meanings and themes and to establish links with the evidence obtained from observations and interviews. The purpose was not only to find evidence of PCMH implementation and chronic disease management processes, but also patterns that would help identify unique or common implementation practices and ways in which PCMH implementation was interpreted and conducted by each site.

\section{Semi-Structured Interviews}

Interviews were the third and final data collection method used as part of this study. This technique enables collection of data that reflect experiences, feelings, attitudes, and opinions that cannot be observed (Schadewaldt et al., 2013). The use of this method helped further explore organizational factors affecting PCMH implementation and chronic disease management efforts through the perspectives of those involved in the process. Interviews were also fundamental to cross-corroborate findings from the previous two methods. They were conducted during the last phase of data collection to avoid any influence of interview responses on the other two phases.

Yin (2003) considers the interview one of the most significant sources of case study information. Within a case study research, interviews take the form of fluid conversations that illuminate the understanding of the phenomenon (Yin, 2003). These conversations are vital to understand the "why", a central focus of this study. Interviews allow the researcher to obtain interpretations of those immersed in the setting of interest (Stake, 1995). This involves the identification of key informants who have experienced 
the phenomenon and can provide researchers, especially in a case study, with insights into the issue of interest and suggest sources to corroborate evidence (Creswell, 2013; Yin, 2003). Patton (2002) considers key informants as the sources of explanation for events that an observer has witnessed but cannot explain.

CHC staff at each participating organization, including leadership and other staff involved in the PCMH implementation and chronic disease management, served a crucial role as key informants. Key informants included: health center leaders (e.g., chief executive officer, chief operating officer, chief medical officer, quality manager, nursing supervisors, site administrator), medical home coordinators, medical home leaders, and other patient support staff, such as a health educator and a nutritionist. While most were recommended by health center leaders serving as gatekeepers throughout the process, several informants were identified during the observation phase. These participants received information about the purpose of the study, the voluntary nature of their participation, and aspects such as confidentiality.

In order to foster conversation flow, while maintaining some form of control over the direction of the interviews, a semi-structured interview strategy was used. Semistructured interviews are guided by a protocol with a series of open-ended questions, but do not impose a fixed set of questions with limited set of responses, allowing for interviewee openness and the emergence of new ideas. This technique provided a way to explore the experience with specific PCMH implementation and chronic disease management aspects from the perspective of the organizational actors.

The interview protocol designed had a total of 18 open-ended questions categorized under five topics. This instrument has been included in Appendix E. 
Interview questions focused on: organizational context and culture, overall PCMH approach, patient-centeredness, PCMH implementation and chronic disease management strategies, leadership and health care team roles, and organizational barriers and facilitators to PCMH implementation and chronic disease management. Whereas the interview protocol provided overall guidance, other questions emerged throughout the interviews as themes and patterns were identified. Most interviews were audio-recorded to facilitate verbatim transcription and analysis. In some cases, to promote trust and openness and avoid any type of pressure or discomfort of key informants, interviews were not recorded. Instead, notes were taken during the interview and documented immediately afterwards.

\section{Member Checking/Validation}

A preliminary within-case analysis process followed data collection at each site to gather general findings and request validation, corroboration, and further input from key informants. Findings and emerging themes from each case were shared with key informants from each participating health center. The input received during this participatory process was anonymously incorporated into findings.

\section{Data Analysis}

Data collection and data analysis followed a structured and systematic inductive approach characteristic of a qualitative design. Instead of making decisions regarding the specific constructs and variables to study upon a set of assumptions and existing theoretical knowledge, the aim of this research was to go to the source to "meet the phenomenon" and gather empirical data without preconceptions. While the data 
collection process led the way to the identification of meanings and themes, a data analysis process was designed to facilitate movement from raw data to discovery of emerging themes (Gray, 2013; Creswell, 2013).

Data analysis took place in four major phases: 1) preparation; 2) development of individual case and context description; 3) within-case analysis; and 4) cross-case analysis. The first three phases occurred at the individual level, for both cases.

Afterwards, both cases were cross-analyzed. As part of the interpretation process, overall assertions, conclusions, and lessons learned were documented. Figure 2 provides a visual summary of the data analysis process described, which followed Creswell's approach to case study research (Creswell, 2013).

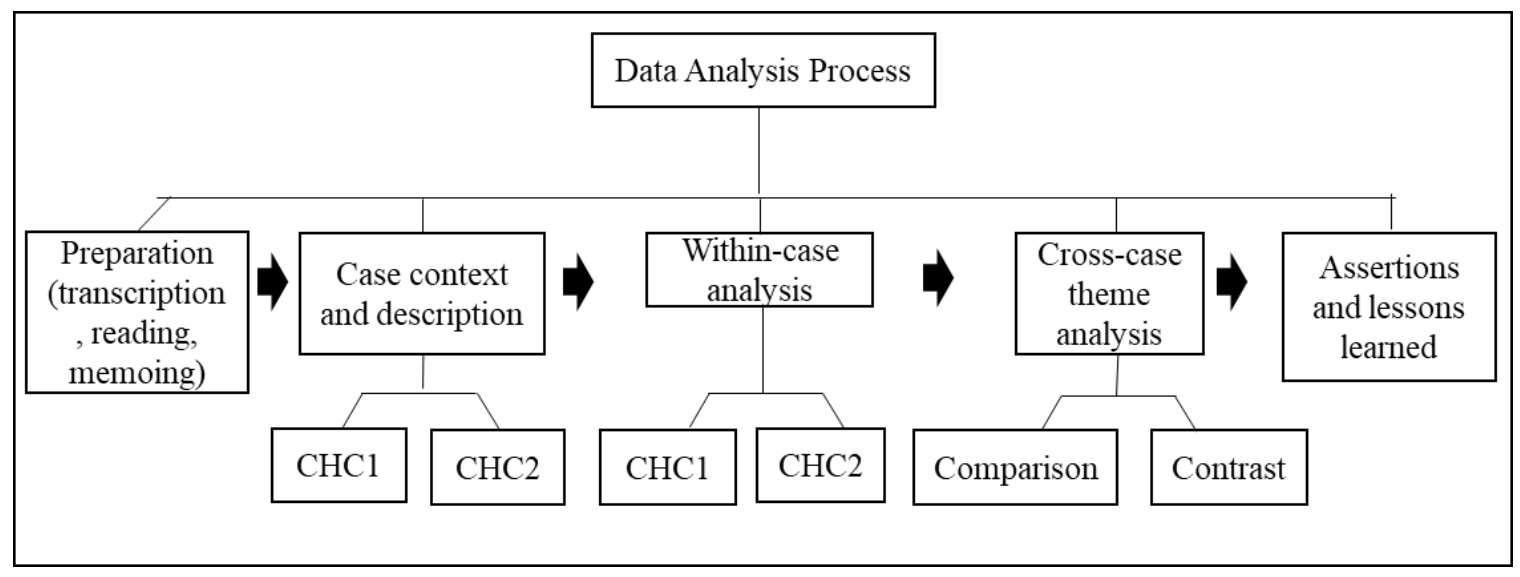

Figure 2. Illustration of the case study/ data analysis process. Adapted from Qualitative Inquiry \& Research Design: Choosing among Five Approaches (p. 209) by J.W. Creswell, 2013, Thousand Oaks, CA: Sage Publications.

During the preparation phase, data was transcribed and systematically documented. Also, a reading and "memoing" process was conducted to get a sense of the database (Creswell, 2013). This involved reading and going over transcripts, field notes, document reviews, texts, and visuals several times to submerge in the details and the 
experience as a whole. During this initial analysis phase, key concepts, outstanding phrases, and emerging ideas were written down in the form of memos. This process took place throughout field note taking, transcribing, and reading. Once raw data were processed and prepared for analysis, data from all sources were integrated to develop detailed descriptions of the health centers' particularities and contexts (Creswell, 2013). This phase mainly involved descriptions of the settings and the evidences found in each case.

A within-case analysis, which involved the identification of themes and categories in each case, was used to develop two individual cases (Stake, 1995; Patton, 2002; Creswell, 2013). Statements and issues discovered in each case were classified into themes, focusing on understanding the complexity of the phenomenon of interest. Themes, concepts, meanings, and experiences emerged from all sources of information collected (Patton, 2002).

Following Creswell's (2013) method, several codes were identified in the beginning and were further expanded as data from all sources was reviewed. Initial themes and categories based on previous literature and theoretical frameworks, were determined a priori to guide the coding process (Creswell, 2013). Nonetheless, the entire analysis process was always open to emerging themes. NVivo12 was used to support the organization of qualitative data and help identify common themes and repetitive patterns. This application supports data analysis through tools that facilitate coding review, text search, and identification of word frequency.

Themes identified were classified under more general categories towards the end of the within-case analysis. Also, statements of significance were discussed under each 
category and theme, placing emphasis on the phenomenon under study. Finding evidence of organizational factors involved in PCMH implementation and chronic disease management was a major key driver throughout this process.

After completing the development of the two cases through the within-case analysis process, both cases were compared and contrasted as part of the cross-case analysis (Stake, 1995; Patton, 2002; Yin, 2003; Creswell, 2013). Similarities and differences under each theme and category were analyzed, leading to the development of assertions and overall conclusions regarding the phenomenon of interest. Common factors involved in PCMH implementation and chronic disease management, as well as major differences in implementation processes, contexts, and organizational dynamics were identified during this process.

\section{Data Interpretation}

Data interpretation occurred throughout the entire data analysis cycle described above, as codes and themes were analyzed in efforts to find larger categories and meanings to explain and reach conclusions regarding the phenomenon of interest (Creswell, 2013). After completion of individual case development and the cross-case analysis process, the research work transitioned from a mainly analytical focus to an interpretive effort. In this phase, moving along the abstraction continuum, the research process transitioned from an inductive approach to a more deductive approach, as theoretical frameworks and relevant literature were introduced to guide interpretation (Creswell, 2013; Yin, 2003).

Due to the importance of understanding the role of context and the health system for this study, several system-level theoretical frameworks were selected to guide this 
research phase. As discussed in the Introduction, the Consolidated Framework for Implementation Research (CFIR), the Expanded Chronic Care Model (ECCM), and the PCMH model itself were used to frame the study's interpretation phase. Findings, emerging themes, and categories developed were interpreted in the light of these theoretical frameworks, as well as relevant literature on the topic. These theoreticallyframed interpretations were then applied in the development of a proposed organizational framework to support successful PMCH implementation and chronic disease management performance among HRSA-funded CHCs.

\section{Quality of the Study and Ethical Considerations}

\section{Strategies to Enhance Quality and Rigor of the Study}

Throughout the research process, several strategies were used to strengthen the quality and credibility of the study. The research design, for instance, incorporated evidence from multiple sources of data as a strategy to corroborate and cross-check findings (Stake, 1995; Yin, 2003; Creswell, 2013). According to Yin (2003), the use of multiple methods provides numerous perspectives on the same phenomenon, increasing construct validity. Findings through one method were cross-checked with findings from another method (Yin, 2003). In addition, a "member-checking" process was added at the end of the data collection process to further support corroboration and validation of findings (Stake, 1995; Patton, 2002; Creswell, 2013).

Data quality also depends in great measure on the rigor with which data was collected. This study followed Yin's logic of replication (Yin, 2003; Creswell, 2013). This is of key importance to this case study, as both cases had to be developed under 
similar conditions, even amid contextual differences. In addition to ensuring that cases selected complied with specific criteria that would provide access to the phenomenon of interest, the study ensured that the same procedures were followed at both sites. To help ensure fieldwork replication fidelity, study protocols and data collection guidelines were used. Moreover, both cases were individually developed following a standardized withincase analysis protocol. While participating $\mathrm{CHCs}$ had differing contexts and approaches to PCMH implementation, their similarity in nature as HRSA-funded CHCs facilitated replication of data collection and analysis procedures.

Another key strategy to enhance data quality was the order in which data collection methods took place. To reduce the risk of potential influence of preconceptions and interpretations from organizational constituents, field observations and documentation review were carried out prior to the interviews. Furthermore, during case development and data analysis, NVivo was used to facilitate the analysis phase, which was important in maintaining objectivity during the analysis of themes. The use of this application further enhanced data quality by supporting the identification of codes and themes, which led to case development.

\section{Ethical Considerations}

Ethical implications were taken into consideration throughout the study. Prior to the beginning of the study, the Florida International University Office of Research Integrity reviewed the study protocol and deemed it Exempt via the Exempt Review process. The researcher also complied with required Responsible Conduct of Research and Human Subjects Research certifications. Site-specific approval was also obtained prior to data collection. Both organizations submitted letters of support. 
During the recruitment process, each health center received an introductory letter with a case statement. In addition, meetings were held with health center leaders to explain the purpose of the study and methods, as well as to respond to existing questions and concerns. Also, an informational letter was developed for distribution among key informants. The letter included details about the study aims, the voluntary nature of participation, the minimal exposure to risks, measures to maintain confidentiality, and contact information for both the researcher and FIU's Office of Research Integrity in case of any ethical concerns regarding the study. CHC leaders met with staff and key informants prior to the study to inform them about the process.

The study did not pose significant health or privacy threats for participants. There were no risks of identification of protected health information (PHI), since this type of data was neither collected or analyzed. The information reviewed, collected, and analyzed focused solely on organizational aspects. There were no interactions with patients and no patient data was reviewed. In addition, no names were used or reported. To ensure health center anonymity, any potential health center identifiers were avoided, including specific location and the use of exact numbers when discussing health center profiles. Specific quotes used to highlight a particular point have been reported without any mentions of participant or health center names.

Throughout data collection, sites were respected and disrupted as little as possible. For instance, non-participant direct observations were conducted without affecting health center operations or patient flow. In addition, as part of efforts to give back to participating sites, a report with findings and conclusions will be shared with each site. Participating organizations will also have access to the framework developed to 
support PCMH implementation and chronic disease management among HRSA-funded CHCs. Study findings, conclusions, and the proposed framework will be formally presented to both organizations.

Chapters 3 and 4 develop findings and results yielded by the data analytic procedures outlined above. Each case is presented separately, whenever appropriate comparisons and contrasts are indicated. Chapter 5 summarizes findings from both cases and offers suggestions for future research and policy. 


\section{Chapter III}

\section{CASE 1}

This chapter presents data analysis and findings resulting from the data collection effort conducted at the first participating community health center (CHC1). Data analysis and ensuing findings are presented as an individual case developed for this participating organization after completion of data collection, which included data collected through observations, document review and individual and group interviews with key informants at $\mathrm{CHC} 1$. This process was facilitated by the CHC's Chief Operating Officer (COO), the main liaison with the organization. After reaching out to the health center's Chief Operating Officer (CEO) in an invitation to participate in the study, the CEO determined the $\mathrm{COO}$, who was also responsible for the Patient Services unit and PCMH implementation, would be the main point of contact and facilitator.

A total of 35 hours of observation were completed at this site, 30 of which took place in public areas, patient waiting rooms, hallways, general service areas, and the registration office. Health center walkthroughs were coordinated with a health center liaison to ensure capture of the manifestation of key aspects of the PCMCH model. The remaining five hours included three hours of observations at the corporate level and two hours observing the community and location. In addition to observations conducted at the site, 37 documents were carefully reviewed as part of this research process. These are summarized in Table 2. In addition, the CHC's website and social network account posts were reviewed. 
Table 2.

Documents reviewed as part of the research process at $\mathrm{CHC} 1$

\begin{tabular}{|l|c|l|}
\hline \multicolumn{1}{|c|}{ Type } & Number & \multicolumn{1}{c|}{ Examples } \\
\hline Samples & 11 & $\begin{array}{l}\text { Sample provider schedules (time slots and reasons for } \\
\text { visits) } \\
\text { Worksheets (Quality Measures, PCMH Record } \\
\text { Review) } \\
\text { Screenshots of unidentified electronic charts } \\
\text { Sample tables with data on compliance with PCMH } \\
\text { factors }\end{array}$ \\
\hline $\begin{array}{l}\text { Patient } \\
\text { communications }\end{array}$ & 10 & $\begin{array}{l}\text { PCMH Roles and Responsibilities (English/Spanish) } \\
\text { Roles and responsibilities related to external referrals } \\
\text { Notice about implementation of the PCMH model } \\
\text { Educational brochures and flyers sampled at site }\end{array}$ \\
\hline $\begin{array}{l}\text { Health Center } \\
\text { information }\end{array}$ & 7 & $\begin{array}{l}\text { List of awards and recognitions } \\
\text { Health center brochure } \\
\text { Organizational charts for multiple areas }\end{array}$ \\
\hline $\begin{array}{l}\text { Mealth Center } \\
\text { policies }\end{array}$ & 4 & $\begin{array}{l}\text { PCMH Interdisciplinary Teams Policy } \\
\text { Notice of Privacy Practices } \\
\text { Same Day Policy } \\
\text { Patient Intake During Emergent or Urgent Care }\end{array}$ \\
\hline
\end{tabular}

The final data collection process included a total of eight semi-structured interviews, seven individual and one group interview with the Medical Home Team, constituted by medical home coordinators, the Medical Home Manager, and the COO. The seven individual key informants interviewed during the field research process included: the CHC's CEO, the COO, the Medical Home Manager, Nursing Supervisor, and three medical home coordinators. In total, close to 7 hours (390 minutes) of interviews were conducted at $\mathrm{CHC}$. Interview duration ranged from 30 minutes to 2 
hours. Table 3 includes the list of questions used as guidelines during interviews with key informants. Each category of questions is aligned with the themes that emerged during data analysis and associated emerging terms.

Table 3.

Case 1 Interview Guiding Questions and Emerging Themes

\begin{tabular}{|c|c|c|c|}
\hline Topic & Guiding Questions & Emerging Themes & Associated Terms \\
\hline $\begin{array}{l}\text { Organizational } \\
\text { culture }\end{array}$ & $\begin{array}{l}\text { 1. Tell me about your } \\
\text { organization. } \\
\text { 2. How would you } \\
\text { describe it? } \\
\text { 3. How would you } \\
\text { define the people } \\
\text { working at the } \\
\text { organization? }\end{array}$ & $\begin{array}{l}\text { Patient-centered } \\
\text { culture } \\
\text { Organizational } \\
\text { culture, leadership } \\
\text { culture, and staff } \\
\text { characteristics }\end{array}$ & $\begin{array}{l}\text { Caring for patients } \\
\text { Patient care } \\
\text { Patient-care vision } \\
\text { Patient- } \\
\text { centeredness }\end{array}$ \\
\hline $\begin{array}{l}\text { Patient } \\
\text { Centered } \\
\text { approach }\end{array}$ & $\begin{array}{l}\text { 4. How would you } \\
\text { define "patient- } \\
\text { centered"? } \\
\text { 5. What makes this a } \\
\text { patient-centered } \\
\text { organization? } \\
\text { 6. What do you do to } \\
\text { maintain the "patient- } \\
\text { centeredness" of the } \\
\text { health center? } \\
\text { 7. Does this focus fit } \\
\text { the essence of your } \\
\text { organization? How? }\end{array}$ & $\begin{array}{l}\text { Need-based care } \\
\text { Comprehensive } \\
\text { services } \\
\text { Organizational } \\
\text { culture, leadership } \\
\text { culture, and staff } \\
\text { characteristics }\end{array}$ & $\begin{array}{l}\text { Patient needs } \\
\text { Patient preferences } \\
\text { Patient first } \\
\text { Address needs } \\
\text { Focus on patient } \\
\text { Comprehensive } \\
\text { services }\end{array}$ \\
\hline $\begin{array}{l}\text { PCMH and } \\
\text { Chronic } \\
\text { Disease } \\
\text { Management }\end{array}$ & $\begin{array}{l}\text { 8. How does this } \\
\text { "patient-centered" } \\
\text { approach you are } \\
\text { describing relate to } \\
\text { your work with } \\
\text { chronic disease } \\
\text { management? } \\
\text { 9. How do you use that } \\
\text { "patient-centered" } \\
\text { strategy to support } \\
\text { chronic disease } \\
\text { management? } \\
\text { (Examples) }\end{array}$ & $\begin{array}{l}\text { Team-based care } \\
\text { Care coordination } \\
\text { Planned care } \\
\text { Self-care support } \\
\text { Patient education } \\
\text { Patient } \\
\text { communication } \\
\text { Patient participation } \\
\text { Quality } \\
\text { improvement } \\
\text { Chronic disease } \\
\text { management }\end{array}$ & $\begin{array}{l}\text { Team huddles } \\
\text { Patient follow up } \\
\text { Tracking } \\
\text { Monitoring } \\
\text { Education } \\
\text { Planned visits } \\
\text { Self-management } \\
\text { Patient compliance } \\
\text { Patient } \\
\text { participation, } \\
\text { involvement } \\
\text { Quality } \\
\text { improvement }\end{array}$ \\
\hline
\end{tabular}




\begin{tabular}{|c|c|c|c|}
\hline & $\begin{array}{l}\text { 10. Describe what your } \\
\text { organization does to } \\
\text { support chronic } \\
\text { disease management } \\
\text { 11. What is and/or how } \\
\text { do you see your role } \\
\text { in this process? }\end{array}$ & & $\begin{array}{l}\text { Self-management } \\
\text { support } \\
\text { Coordinated care } \\
\text { Communication } \\
\text { Shared visits } \\
\text { Group education } \\
\text { Diabetes } \\
\text { management } \\
\text { Blood pressure } \\
\text { Patient goals }\end{array}$ \\
\hline $\begin{array}{l}\text { PMCH } \\
\text { Implementation }\end{array}$ & $\begin{array}{l}\text { 12. Tell me about your } \\
\text { experience } \\
\text { implementing the } \\
\text { Patient-Centered } \\
\text { Medical Home model } \\
\text { 13. What were some of } \\
\text { the key changes you } \\
\text { had to go through? } \\
\text { 14. The model has } \\
\text { several components. } \\
\text { Can you tell me } \\
\text { about how your } \\
\text { organization covers } \\
\text { these components? }\end{array}$ & $\begin{array}{l}\text { Quality } \\
\text { improvement } \\
\text { Approach to } \\
\text { implementation } \\
\text { Comprehensive } \\
\text { care } \\
\text { Patient support } \\
\text { Culturally and } \\
\text { linguistically } \\
\text { appropriate services } \\
\text { Patient participation } \\
\text { in care } \\
\text { Access to care } \\
\text { Team-based care } \\
\text { Care coordination } \\
\text { practices } \\
\text { Planned care } \\
\text { Use of technology } \\
\text { Population health } \\
\text { management } \\
\text { Organizational } \\
\text { structure } \\
\text { Leadership culture } \\
\text { Performance and } \\
\text { quality } \\
\text { improvement } \\
\text { Strategic } \\
\text { partnerships }\end{array}$ & $\begin{array}{l}\text { Quality } \\
\text { improvement } \\
\text { Patient goals } \\
\text { Resources } \\
\text { Organizational } \\
\text { model } \\
\text { Staff resources } \\
\text { Funding } \\
\text { Management } \\
\text { resources } \\
\text { Team-based care } \\
\text { Health care teams } \\
\text { Medical Home } \\
\text { Coordinators } \\
\text { Referral } \\
\text { Coordinators } \\
\text { PCMH structure } \\
\text { Medical Home } \\
\text { Team } \\
\text { Team huddles } \\
\text { Partnerships } \\
\text { Multidisciplinary } \\
\text { care } \\
\text { Languages } \\
\text { PCMH assessment } \\
\text { Performance } \\
\text { improvement } \\
\text { Access to care } \\
\text { Patient load/wait } \\
\text { Walk-in/Same Day } \\
\text { Technology/EHR } \\
\text { Continuity of care } \\
\text { Patient portal } \\
\text { Population health } \\
\text { Patient data }\end{array}$ \\
\hline
\end{tabular}




\begin{tabular}{|c|c|c|c|}
\hline & & & $\begin{array}{l}\text { Comprehensive } \\
\text { services } \\
\text { Coordination of } \\
\text { care } \\
\text { Patient } \\
\text { participation } \\
\text { Support services } \\
\text { Specialists }\end{array}$ \\
\hline Facilitators & $\begin{array}{l}\text { 15. Let's talk about the } \\
\text { things or factors you } \\
\text { believe contributed to } \\
\text { the implementation } \\
\text { of the PCMH model } \\
\text { (anything that } \\
\text { facilitated the } \\
\text { process). Which } \\
\text { organizational } \\
\text { attributes do you } \\
\text { think contributed to } \\
\text { this process? } \\
\text { 16. Tell me about your } \\
\text { roles in this process. } \\
\text { 17. What can you say } \\
\text { about the role of the } \\
\text { health center's } \\
\text { leadership in this } \\
\text { process? }\end{array}$ & $\begin{array}{l}\text { Patient } \\
\text { communication and } \\
\text { education } \\
\text { Patient support } \\
\text { Culturally } \\
\text { appropriate services } \\
\text { Patient participation } \\
\text { in the care process } \\
\text { Comprehensive } \\
\text { care } \\
\text { Access to care } \\
\text { Team-based care } \\
\text { Care coordination } \\
\text { Use of technology } \\
\text { to facilitate patient } \\
\text { care } \\
\text { Population health } \\
\text { management } \\
\text { Organizational } \\
\text { structure } \\
\text { Leadership culture } \\
\text { and staff } \\
\text { characteristics } \\
\text { Performance and } \\
\text { quality } \\
\text { improvement } \\
\text { Strategic } \\
\text { partnerships } \\
\text { Facilitators } \\
\text { Approach to PCMH }\end{array}$ & $\begin{array}{l}\text { Medical Home } \\
\text { Coordinators } \\
\text { PCMH structure } \\
\text { Communication } \\
\text { system } \\
\text { Appointment } \\
\text { system } \\
\text { EHR } \\
\text { Technology } \\
\text { Support staff } \\
\text { Patient } \\
\text { communication } \\
\text { Care coordination } \\
\text { Leadership } \\
\text { Recognition } \\
\text { Referral } \\
\text { Coordinators } \\
\text { Team-based care } \\
\text { Specialists } \\
\text { Quality } \\
\text { improvement } \\
\text { Partnerships } \\
\text { Enhanced access } \\
\text { Cultural diversity } \\
\text { Patient-centered } \\
\text { vision } \\
\text { Relationship with } \\
\text { patients } \\
\text { Team support } \\
\text { Team players } \\
\text { Leadership } \\
\text { monitoring } \\
\text { Resources }\end{array}$ \\
\hline Barriers & $\begin{array}{l}\text { 18. Now, let's think } \\
\text { about barriers or } \\
\text { obstacles } \\
\text { encountered along }\end{array}$ & $\begin{array}{l}\text { Barriers to } \\
\text { implementation } \\
\text { Patient } \\
\text { communication }\end{array}$ & $\begin{array}{l}\text { Managed care fees } \\
\text { Costs, expenses } \\
\text { Limited funding } \\
\text { Resources invested }\end{array}$ \\
\hline
\end{tabular}




\begin{tabular}{|l|l|l|l|}
\hline & $\begin{array}{l}\text { the way. Which } \\
\text { factors or issues do } \\
\text { you believe make it } \\
\text { difficult to } \\
\text { implement the } \\
\text { components of this } \\
\text { model? }\end{array}$ & $\begin{array}{l}\text { Team-based care } \\
\text { Care coordination } \\
\text { Patient support }\end{array}$ & $\begin{array}{l}\text { Patient non- } \\
\text { compliance } \\
\text { Complex } \\
\text { processes } \\
\text { Referrals } \\
\end{array}$ \\
& & Communication \\
& & with patient \\
& & Transportation \\
& & Requirements \\
& & Staff recruitment \\
& & Staff shortage, \\
& & limited staff \\
& & Workload \\
\hline
\end{tabular}

In what follows, data are presented according to the themes that naturally emerged from observations, document review, and interview data in response to the questions guiding the study. Rather than grouping data according to the structured format of the questions, the choice was made to allow the data to flow as it naturally emerged from the dynamic exchanges that occurred while interviewing or during observations. A total of 19 themes emerged from the analysis of the data collected through the three procedures discussed. These themes were used as guidelines to develop Case 1 and Case 2, presented in the following chapter. This chapter begins with an overview of $\mathrm{CHC} 1$, a description of the site, a summary of emerging themes identified in the data collected and an integrated discussion of findings by theme. The last section of this chapter summarizes the key findings and major challenges and facilitators identified at this $\mathrm{CHC}$.

\section{Community Health Center 1 (CHC1) Overview}

Founded over four decades ago, this CHC, one of the 17 HRSA-funded CHCs serving the South Florida region, served nearly 50,000 patients in 2017. Most patients were low-income individuals from racial/ethnic minority groups (HRSA, 2018c). In 2017, more than a third of patients served by this CHC were uninsured and nearly one- 
fifth were Medicaid/CHIP beneficiaries. Nearly 20\% of this CHC patients (nearly 9,000) had hypertension and over 10\% had diabetes (close to 5,000). In 2017, close to onefourth of hypertensive patients and one-fourth of diabetic patients were uncontrolled (HRSA, 2018c).

\section{Site Description}

Observations took place at the CHC's main site, a four-story building of nearly 50,000 square feet of office space located in a highly commercial and densely populated urban community. Considered a significant health care and employment resource for the community, this organization had nearly 60 providers across its multiple sites at the time of the study. According to the Centers for Medicare \& Medicaid Services (2018), in 2018 there were over 2,700 primary care providers in the CHC's service area and neighborhoods within 15 miles of the site's location. These included over 600 general and family practitioners, more than 1,600 internists, close to 200 gynecologists, and 180 pediatricians.

The first visit to the health center took place during a weekday winter morning. It was hard to see the glass door entrance to the four-story building due to the multiple patients either arriving, leaving, or waiting to be picked up. Navigation around the facility was a bit challenging at first, mainly because of the way the building is structured and departments are physically arranged. The health center's multiple departments were distributed throughout the four floors, mostly behind closed doors. Every department had its own office space in the building, each with separate waiting areas. It seemed initially confusing to determine where to go, but there was a sign indicating that patients must register on the third floor. 
As arranged by the health center liaison, most observations were conducted in the Endocrinology/Diabetes Management Department. While this office had a small 11-seat patient waiting room, there were about six exam rooms behind the front desk reception door. The area was always clean and bright. It was generally a quiet area with not much taking place, except front desk staff actively calling and receiving calls from patients. Most of the patients visiting the clinic during the observation period were middle-aged, mainly Hispanic and African American.

\section{Emerging Themes}

Nvivo and line-by-line analysis were used to facilitate the examination of emerging themes. After transcribing, documenting, and organizing data collected, the texts produced were imported to Nvivo, one source at a time. A line-by-line text analysis was conducted to confirm the themes identified. Appendix G includes a list of emerging themes by method and associated key terms found. The following sections include a discussion of emerging themes, organized into six major categories, as shown in Table 4.

\section{Table 4.}

Categorized emerging themes for Case 1

\begin{tabular}{|l|l|}
\hline \multicolumn{1}{|c|}{ Categories } & \multicolumn{1}{c|}{ Emerging themes } \\
\hline $\begin{array}{l}\text { 1. Patient-centered, need-based } \\
\text { care }\end{array}$ & $\begin{array}{l}\text { Comprehensive care } \\
\text { Patient support } \\
\text { Culturally and linguistically appropriate services } \\
\text { Patient participation in the care process } \\
\text { Access to care }\end{array}$ \\
\hline $\begin{array}{l}\text { 2. Coordination and integration } \\
\text { of care }\end{array}$ & $\begin{array}{l}\text { Team-based care } \\
\text { Care coordination practices } \\
\text { Planned care }\end{array}$ \\
\hline
\end{tabular}




\begin{tabular}{|l|l|}
\hline $\begin{array}{l}\text { 3. Chronic disease } \\
\text { management, self-care support, } \\
\text { and education }\end{array}$ & $\begin{array}{l}\text { Chronic disease management and self-care support } \\
\text { Patient communication and education }\end{array}$ \\
\hline 4. Technology integration & $\begin{array}{l}\text { Use of technology to facilitate patient care } \\
\text { Population health management }\end{array}$ \\
\hline $\begin{array}{l}\text { 5. Organizational system and } \\
\text { culture }\end{array}$ & $\begin{array}{l}\text { Organizational structure } \\
\text { Organizational culture, leadership culture, and } \\
\text { staff characteristics } \\
\text { Learning climate, performance and quality } \\
\text { improvement } \\
\text { Strategic partnerships }\end{array}$ \\
\hline $\begin{array}{l}\text { 6. Medical Home } \\
\text { implementation }\end{array}$ & $\begin{array}{l}\text { Approach to PCMH implementation } \\
\text { Barriers to implementation } \\
\text { Implementation facilitators }\end{array}$ \\
\hline
\end{tabular}

\section{Patient-Centered, Need-Based Care}

\section{Comprehensive and Integrated Care}

Field observations conducted at waiting areas, hallways, and other general areas

of the facility for 30 hours, including a walkthrough guided by the Medical Home Manager, provided an opportunity to witness multiple services provided across the organization. These services were available onsite, following a "one-stop shopping" model. The patient was able to access many of the services needed in the same facility, without having to move from building to building or see other providers. These included primary care for all life cycles (pediatric, adult, geriatric), preventive screenings, specialty care, patient support services, immunization, chronic disease management, behavioral health, oral health, vision care, pharmacy, radiology, and laboratory.

The Pediatrics and Pharmacy areas could be immediately found on the first floor. Adult primary care areas were on the third and fourth floors. There were also several specialties, such as cardiology and endocrinology, and an obstetrics/gynecology department, which was at the time under an expansion project in response to the 
increased demand. Most of these services were found on the third floor, where the main registration area was also located. Patient brochures examined provided additional information on the services offered, which have been included in Table 5.

\section{Table 5.}

Health services provided by $\mathrm{CHC} 1$

\begin{tabular}{|l|l|}
\hline $\begin{array}{l}\text { Preventive and } \\
\text { Primary Care }\end{array}$ & $\begin{array}{l}\text { Preventive screenings, General Medicine, Internal Medicine, } \\
\text { Pediatrics, Developmental screening, Geriatric care, Chronic } \\
\text { disease management, Obstetrics/Gynecology, On-site laboratory, } \\
\text { Pharmacy, STD Testing/ Treatment/ Prevention, Immunization }\end{array}$ \\
\hline $\begin{array}{l}\text { Behavioral } \\
\text { Health }\end{array}$ & Mental health counseling, Psychiatry, Psychotherapy, Social Work \\
\hline $\begin{array}{l}\text { Oral Health, } \\
\text { Vision, and } \\
\text { Hearing }\end{array}$ & $\begin{array}{l}\text { Preventive and basic dental services, Ophthalmology and } \\
\text { Optometry, Vision \& Hearing Screenings }\end{array}$ \\
\hline Specialty Care & Cardiology, Endocrinology, Podiatry, Specialist referrals \\
\hline HIV/AIDS & $\begin{array}{l}\text { HIV/AIDS Testing, HIV/AIDS Counseling, immune support } \\
\text { programs, HIV Outreach Services, Case Management }\end{array}$ \\
\hline $\begin{array}{l}\text { Enabling/ } \\
\text { Patient Support } \\
\text { Services }\end{array}$ & $\begin{array}{l}\text { Health Education, Affordable Care Act eligibility assistance, case } \\
\text { management, patient navigation, family planning, outreach } \\
\text { services, Women, Infants and Children Program (WIC), } \\
\text { Transportation }\end{array}$ \\
\hline
\end{tabular}

Information about the comprehensiveness of services was also corroborated through interviews and several informal conversations with the $\mathrm{COO}$, the Medical Home Manager, Medical Home Coordinators, and the nursing supervisor at the Endocrinology/Diabetes Management Department. Interviews confirmed that all these services are available internally. The $\mathrm{COO}$ indicated that the health center had specialties that not every CHC has. The Diabetes Management Unit Nursing Supervisor believes this is an important strategy to address patients' needs and enhance access to care. She explained that the $\mathrm{CHC}$ established a system that incorporates specialists that are key to 
chronic disease management and address multiple health needs. These specialists were not only available on-site but are also affordable for patients.

As clarified by the $\mathrm{COO}$, there is no need to create referrals for internal services. Through an appointment template in the electronic system, the staff scheduled appointments with other departments, including behavioral health. If the appointment is needed the same day, the patient can be transferred to the service department or unit. If the patient needs lab work done right away, there are three labs onsite and the patient can get the labs done at the moment. One of the laboratories shared space with the Endocrinology/Diabetes Management department and patients were sent directly for labs when needed.

This is part of the organization's focus on patient-centeredness. According to the $\mathrm{COO}$, in the health center's service delivery model, "everything revolves around the patient." She further explained:

"All the care a patient needs must be resolved for the patient. It's not a matter of putting the patient in the room and just seeing patients. It's a matter of making sure that all the needs are addressed for that patient. If the patient needs to get lab work, see a social worker, get Medicaid, or any type of social services, that's what we call patient-centeredness."

\section{Patient Support and Enabling Services}

A fundamental part of the health center's approach to comprehensive care and patient-centeredness was the availability of patient support and enabling services to help minimize existing barriers to care. Observations collected across the site included signs 
and announcements indicating that services were provided regardless of patients' ability to pay or health care coverage status. In addition, the organization addressed financial barriers through health care coverage eligibility and enrollment assistance, payment plans, and income-based sliding fee discounts aligned with the most recent federal poverty guidelines. There were several signs at the site announcing the availability of sliding fee discounts. In addition, the discount scale was available for patient review at Registration. One staff member in the area observed was seen completing a sliding fee discount evaluation for one of the patients. Also, during the observation period, one of the front desk staff support set up a payment plan for a patient who needed help with his copay.

During an interview, the COO indicated that the health center also had a pharmacy patient assistance program for medications that are expensive and offered vouchers for medications. She added that, to support patients without health coverage, the Outreach and Enrollment staff qualified patients for Medicaid and filled out the Medicaid application for those eligible. This team also helped patients get coverage through the Health Insurance Marketplace. According to the COO, this unit also helped patients with Supplemental Nutrition Assistance Program (SNAP) applications.

As confirmed through the review of organizational documents, policies, and brochures, the $\mathrm{CHC}$ also supported patients through social work, case management, health education, transportation, and translation and interpretation services. There were also several messages and announcements available throughout the facility indicating the availability of translation and interpretation services. 


\section{Linguistically and Culturally Appropriate Services}

As noticed during the 30 hours of onsite observations, the health center made an effort to ensure that patients received services in the language of preference or had access to a system that allowed them to communicate in their preferred language. This was evident throughout the site, where there were informational wall displays in all three most frequently heard patients' languages at the site. Further, the review of organizational communications and print materials indicated that most were available in English, Spanish, and Haitian Creole, which the patient liaison acknowledged represented the three most common patient ethnicities. Observations at the site and informal conversations with patients confirmed that there was always a Spanish-speaking staff member available and some staff members, particularly providers, spoke Creole. For other languages, as indicated in an announcement available in the registration area, the CHC provided interpretation services using the STRATUS system, which offered interpretation for up to 20 different languages. The availability and provision of these services were noted further when reviewing the unidentified electronic patient chart samples provided by the organization. The latter revealed that communications needs were documented in the patient's record. These include language of preference, language the patient best reads and speaks, if an interpreter is needed, or if sign language is needed.

In addition to language, the organization paid attention to cultural competence and respect for diversity. This was further documented by observations and interviews that highlighted the patient support provided by health care team members and their high level of understanding and respect for the patient's needs, culture, values, and preferences. Throughout the time spent at the site, as well as during informal 
conversations and interviews, it became evident that staff members at different levels of the organization demonstrated knowledge and understanding about different cultural behaviors, beliefs, and preferences. Moreover, as observed during the site visit and through the examination of the health center's webpage, the staff was culturally diverse. According to the Nursing Supervisor, this was a key success factor for both her department and the organization. Details about the patient's beliefs, preferences, and values were also documented in the patient's record.

\section{Patient Participation in the Process of Care}

The health care team encouraged patient involvement in the process of care and health care decisions. During the observed "team huddle", a brief daily meeting taking place at the start of each day among members of the health care team, the leading physician pointed out the importance of ensuring patients understood the importance of committing to their own care and engaging in his/her own care. As reviewed, forms signed by patients when referred to an external provider not only stated this responsibility, but also documented patients' knowledge about the referral and why he or she was being referred. As the physician expressed during the "huddle", "this is an important part of engaging the patient."

The PCMH program policy reviewed stated the health center's commitment to involving the patient in decisions about his/her health and health care. This policy document also stated health center and patient roles and responsibilities in the PCMH model as they worked together to keep the patient healthy. According to the document, patients were encouraged to act as full partners in the care process, committing to appointments, participating actively during each visit, helping providers coordinated with 
other providers, following the plan agreed, and providing feedback to help the provider and the organization improve.

Medical home coordinators interviewed while the researcher conducted observations and interviews at the site, discussed how the organization engaged patients with chronic diseases in multiple individual and group educational sessions to help them improve self-management practices. The staff confirmed that patients were also encouraged to participate in different community events throughout the year. As observed in health center social network messages, patients were frequently invited to participate in health fairs, free breast cancer screenings, back-to-school fairs, and family days, among others.

\section{Access to Care}

Practices and strategies in place by the $\mathrm{CHC}$ to enhance access to care included extended hours of operation, acceptance of walk-in patients and same day appointment, and "after hours" care. Their implementation was observed during site visits. For example, hours of operation were displayed across the site, including sings on doors and screens; the website also displayed this information. Regular health center services were available from 7:30 am to 5:00 pm on weekdays. Additionally, primary care, pediatrics, and pharmacy services were available on Saturdays. Specialty care services were only available weekdays until 4:00 p.m.

The CHC made an effort to ensure availability of access to care or clinical advice 24/7 through a phone line service. There were several bulletin boards across the site with instructions on how to access services after regular hours, including a number to reach a medical provider after-hours. Medical home coordinators interviewed indicated that 
providers and medical home coordinators could also be reached through the patient portal. In addition, the CEO explained that patient ID cards had direct contact information for both the physician and the patient's medical home coordinator, including this staff's cell phone number.

During the group interview, the Medical Home Manager affirmed that appointments could be scheduled in person, by phone, or through the patient portal, and that these were usually available in one to two weeks, but a patient with a health issue could get a same-day appointment. As observed in the documents reviewed, as part of its PCMH certification, the $\mathrm{CHC}$ was required to reserve appointment slots for walkin/same-day patients. The organization established a Same Day Policy as part of the Appointment Scheduling Policy to enhance the timeliness and efficiency of its services. According to the policy reviewed, an intake nurse must assess the patient and assign appointment slots in-between scheduled patients. At the observed Endocrinology/ Diabetes Management Department, medical home coordinators assessed the situation and accommodated patients according to their need.

In spite of the effort made by the CHC to accept walk-ins and its workings, as explained by those interviewed, observations suggest that, at times, accepting "walkin"/"same-day" patients represented a challenge to the efforts to enhance the timeliness of visits. In the area observed, wait time for scheduled patients ranged from 15 minutes to up to an hour, depending on the number of people scheduled that day and staff availability. However, one day during the observation period, the office was overcrowded with scheduled patients, same-day patients, and patients who could not be seen the previous day. In the waiting area, some patients expressed that the wait could be very 
long for a patient without a scheduled appointment. Further commenting on scheduling challenges, the $\mathrm{COO}$ and the Medical Home Manager observed that patient load would be exceptionally high the next available day after a holiday, if the health center was closed on Saturday, or the day the provider comes back from a day off or vacations.

\section{Coordination and Integration of Care}

\section{Care Coordination}

One key opportunity throughout the observation period was being able to witness the work of Medical Home Coordinators (MHCs), a network of licensed practical nurses (LPNs) responsible for ensuring patients receive the care needed. Each MHC was assigned to one or more departments and a group of patients for whom they ran the entire care coordination process. The observation process included a site walkthrough that allowed direct observation of the dynamics behind the MHCs work.

During the walkthrough observations, MHCs were working non-stop at their desks reviewing patient charts, calling patients to remind them of either appointments or tests, pre-planning patient visits, and responding to calls or email messages from patients. One female MHC called a patient on the phone to let him/her know that she had just noticed in the electronic system that his/her A1c was due. She told the patient to stop by to get the lab work done so the doctor could have it available in the next visit. MHCs also collected data on the patients confirmed for an upcoming visit and discussed the cases with the members of the health care team during daily "team huddles".

In addition to the $\mathrm{COO}$ and the Medical Home Manager, two MHCs were interviewed during the research period. According to one of the MHCs interviewed, the MHC served as "the main liaison between the patient and the providers." If patients 
needed to have consultations in between appointments or had a particular need, they could reach out any time to their MHC. As described by the MHC, they also assess each patient's situation before their visit and "make sure that patients understand what to do to take care of their conditions at home." She added that if there is a list of patients with a condition like high blood pressure who must be seen, MHCs coordinate with the patients and explain what and why they need to come in for. As expressed by the leading physician in the Endocrinology/Diabetes Management department during the team huddle observed, "some patients may need more attention and 'babying' than others."

According to the $\mathrm{COO}$, this process is important "not only in complying with the requirements of being a medical home, but in assisting the patient in achieving the goals for their conditions." This is why, as she explained, MHCs contributed to a successful implementation of the PCMH model. In addition to MHCs, the two MCHs interviewed observed that the care coordination process was aided by a network of Referral Coordinators (RCs) and that each provider had a referral coordinator assigned to take care of external referrals for that provider. One $\mathrm{MCH}$ noted that, "their primary responsibility is to make the appointment for the patient and find out whether the patient needs an authorization from the health insurance and get the authorization." She added that the RCs also called the specialist's office to make sure the report was received before closing the loop on the referral.

The COO, Medical Home Manager, and MHCs participating in the group interview explained that MHCs coordinated onsite services and worked closely with RCs. MHCs ensured that appointments coordinated by RCs worked for their patients, that patients complied with appointments, and they brought back a report from the external 
provider. This could be a very complex task because of the numerous referrals submitted daily. According to the health center's CEO, CHC providers could issue 60,000 referrals every year. Lastly, no discrepancies were observed between site observations conducted during the walkthrough and information on the purpose and work objectives of the $\mathrm{MCHs}$, as described by the $\mathrm{COO}$ and the Medical Home Manager.

\section{Planned Care}

During the period of observations at the site and during the course of the walkthrough conducted, MHCs were seen assessing each scheduled patient's chart prior to the visit to review their situation, check any tests due, and what they were coming for to ensure they were ready for the visit. MHCs were continuously evaluating patient charts and calling patients to arrange for any procedures, tests, or preventive screenings pending. As explained by MHCs interviewed, pre-planned visits were discussed at the start of each day during "team huddles", which helped maximize visit time and ensured patient needs were addressed.

During the huddle observed, the MHC presented the number of patients confirmed for the day and went over specific situations. For instance, the physician reminded the team of the importance of referring patients for colorectal cancer screening. He also reviewed scheduled patients' charts on his computer and pointed to some cases in need of attention. As evidenced by the documents reviewed, including the Interdisciplinary Teams Policy and the structure of the team huddle agenda, these short daily meetings were expected to cover patient care gaps, challenging patients or situations, "walk-in" slots, any miscategorized appointments, missing vaccines or tests, 
aspects related to patient flow and work flow efficiency, and any particular patient case or health risk.

\section{Team-Based Care}

Team-based care was another key feature of the PCMH model observed. Throughout the observation, different members of the team could be seen working independently on patient issues. Furthermore, participation in the team huddle provided an opportunity to observe the group's social interactions and overall working dynamics of the health care team. The observed team consisted of six members which included the leading physician, the head nurse/supervisor, the MHC, medical assistant, scribe (LPN), and the dietitian. The dietitian, nurse, and physician seemed to collaborate regularly on patients' cases and care plans.

In addition to the above observations and an important component of the triangulation of methods employed in developing the case study, a review was conducted of the CHC's Medical Home Interdisciplinary Teams Policy. The policy stated that these teams were created to support comprehensive and coordinated care. It established that every patient must receive care with the same provider and interdisciplinary team. This document also indicated the required composition of the team: the provider, the licensed practical nurse (LPN)/scribe, medical assistant, medical home coordinator, referral coordinator, and behavioral health coordinator. As further confirmed by the MHCs and the COO during interviews, each MHC was usually in charge of two to five providers each. For example, one of the MHCs interviewed had been assigned to two primary care providers and their residents. 
During the above interview with the COO, she explained that, when the health center's leadership looked at the PCMH model and its requirements, they knew it would be difficult to have just one person take care of all the needs of the patient. She added, "a lot of sites have just the doctor and the medical assistant, which doesn't work because this is a multidisciplinary approach." This was the reason provided by the COO about the decision to incorporate MHCs to the team, which led to the hiring of LPNs for this position. According to the CEO, $10 \mathrm{MHCs}$ and $15 \mathrm{RCs}$ support the process. The $\mathrm{COO}$ added that the health center's CEO had many times expressed that this $\mathrm{CHC}$ could be the one with the largest support staff in a health care team. The COO confirmed that there were at least five people in each interdisciplinary team. This health center leader stressed, "in order to be able to provide comprehensive care and coordinated care, you need this type of support."

\section{Chronic Disease Management, Self-Care Support, and Education}

\section{Chronic Disease Management and Self-Care Support}

The Nursing Supervisor at the observed department, the COO, and the department's MHC confirmed during interviews and informal conversations that every patient with diabetes was seen at the observed department and that diabetes management was a major focus of this area. A poster on the editorial board of this department's conference room highlighted a presentation on some of the organization's accomplishments in diabetes management. For instance, the organization was able to increase the proportion of diabetic patients with an annual eye exam. Observations conducted while in the field at this Center site supported the staff's explanations and 
descriptions of their work. The work of the MHCs, the daily interactions between health care team members, and interactions during the "team huddle" evidenced the department's ongoing focus on maintaining control of these conditions and supporting the patient in this process. The members of the health care team monitored, tracked, and followed up with patients on an ongoing basis.

As expressed by the department's Nursing Supervisor during an interview, "this is not just any specialty care office." She pointed out that this health center's chronic disease management practice consisted of a team-based integrated and continuing approach. Moreover, she explained that the department's work was guided by the patient care vision of the leading physician and an in-depth understanding of patients' needs and beliefs. In addition to providing health care, she indicated that the team focused on addressing different barriers for patients' improvement, such as helping with access to medicines and food and assisting with health insurance plan issues. The team also established a special relationship with patients, which the Nursing Supervisor highlighted during the interview as a key factor for the success of the model within the department. "Regardless of the waiting time, patients want to come back," she expressed.

This Nursing Supervisor highlighted the onsite integration of specialists needed to support their effort on chronic disease management and their improvement, which were important responsibilities of this department. This integration required having at the site important partners in achieving these goals to include the endocrinologist, optometrist, ophthalmologist, podiatrist, dietitian, and cardiologist. She explained that, being on site, these providers were both accessible and affordable to patients. Previously, the provider 
would refer to an external optometrist, but patients would not go because they could not afford the costs.

The COO indicated that the organization had been developing strategies to reach HRSA and Healthy People 2020 (HP2020) goals for multiple chronic conditions, mainly diabetes and hypertension. She indicated that the goal was to continue reducing A1c levels to less than $9 \%$. At the time the research was conducted, the COO reported that the proportion of diabetic patients with A1c levels below $9 \%$ was $22 \%$. She expressed that, while the CHC was below its 25\% goal, the CHC wants to reach HP2020's $16 \%$ target. Even though this target had not been achieved, both the health center's administration and staff seemed keen on their need to continue improving toward their target and addressing the barriers and limitations they faced.

Interviews with MHCs, the COO, and the Nursing Supervisor confirmed the use of patient education as a strategy to increase awareness and knowledge about the conditions, self-monitoring, disease control, and wellness. The COO explained that, as part of its hypertension management program, the $\mathrm{CHC}$ had implemented an in-home self-monitoring program through a grant from the American Heart Association. Through this program, patients with hypertension received free blood pressure machines and support from a nurse navigator. In addition, as explained by MHCs, the health center provided small-group classes in different languages where they discussed symptoms, taught patients to monitor blood pressure, and educated about proper medication use and the importance of diet and physical activity. In addition, the Endocrinology Department organized a combined class for patients with diabetes and blood pressure. The COO added that the $\mathrm{CHC}$ also conducted shared medical visits and group education. 
PCMH documents and computer screenshot samples shared by the $\mathrm{CHC}$ revealed some of the organization's electronic health record system (EHR) capabilities, including tools for self-care support and documentation of patient engagement, compliance with recommendations, commitment to appointments, and treatment adherence. The system was also used to document educational interventions such as counseling on regular physical activity and nutrition and participation in group education and support groups. When reviewing the document on the CHC's compliance with PCMH standards, it was observed that the $\mathrm{CHC}$ also documented patient preferences, functional and lifestyle goals, barriers to meeting these goals, and self-care plans.

\section{Patient Communication and Education}

There were several different publications and announcements available in waiting rooms, including educational and health center brochures, printed organizational policies regarding privacy and patients' rights and responsibilities, as well as educational print materials from other organizations, such as pharmaceutical companies. There were also educational posters on the walls about topics such as flu symptoms and prevention, hand hygiene, zika virus symptoms, and heart health. In addition, screens displayed information on general health issues, health center services, and ads from pharmaceutical companies. Despite concerns expressed on improving diabetes and hypertension management no messages about diabetes, hypertension, or other related conditions were displayed on posters posted throughout the public spaces observed. On the other hand, information about organizational policies and services, such as the availability of sliding fee discounts and interpretive services were well displayed on bulletin boards across the organization. 
The document review process included the examination of patient communication materials shared by the health center liaison, materials available at the waiting room, and contents and materials available through the health center's website. These materials included communications regarding patients' rights and responsibilities and health center and patient PCMH roles, as well as flyers about educational events such as labor classes available through Healthy Start. During a total of 30 hours of field observation at this site, no one was seen reading the posted material. The organization's website, also reviewed as part of this process, included educational materials on a variety of topics: HIV awareness and testing, nutrition, healthy lifestyles, mental health, smoking cessation, tips for taking medications, and different quality-related recognitions and awards. In addition, as observed, the organization had an active social media network account where it posted information about health center and community events, accomplishments, preventive recommendations, and current health topics.

During the group interview, the $\mathrm{COO}$ indicated that educational contents displayed throughout the building were chosen based on currently identified patient need, and different performance improvement measures periodically established by the health center, such as blood pressure and overuse of antibiotics. She underlined that these communications were displayed in a standard way across all delivery sites. Moreover, she explained that the CHC assessed patient's health literacy and educational levels through the EHR and developed materials related to high risk areas, such as medication management, at an eight-grade level. However, after examining other general patient information documents using the Flesch-Kincaid scale, a readability analysis tool, only 
two were found to be at an eight-grade level or below. Others scored over 10 on the Flesch-Kincaid scale.

\section{Use of Technology to Facilitate Patient Care and Population Health Management}

Seven total days of observations of the organization's processes, interviews with staff members and documents review showed the prominent use of technology as a tool for practice management, patient care, and care coordination. Throughout the 30-hour period observing work dynamics on site and a walkthrough, front desk staff and registration staff were seen accessing patient information through a practice management system. They were able to check patients in and out through this system, as well as assess patients' eligibility for sliding fee discounts. Observations and information obtained from interviewees confirmed that all health center areas were connected to one network. For instance, a patient asked the front desk staff if she would be able to see the gynecologist once registered with the Endocrinology department. The staff responded that she could stop by the Gynecology department and they would immediately find her in the system. The COO explained during an interview that all patient information was available within one single network and shared among all units and offices. She explained that the staff made appointments for any internal service, including behavioral health, onsite specialized care, obstetrics/gynecology, among others.

During the observation, MHCs could be seen actively looking up and reviewing patients' charts in the EHR in order to pre-plan patient visits. In addition, during the "team huddle" observed, the leading physician reviewed several charts on his laptop and discussed several patient situations with his team. Interviews with MHCs further confirmed the active use of the system as part of the care coordination process. Through 
the EHR, they were able to track and monitor the patient and assess any gaps in care. The $\mathrm{COO}$ also shared that, through health information exchange agreements with a few local hospitals, providers received information about patients who visited the emergency room and were admitted to the hospital, which enabled the $\mathrm{CHC}$ to follow-up with the patient and ensure continuity of care.

MHCs interviewed pointed out that the EHR also helped the health care team maintain ongoing communication with patients. According to one of the MHC's, through the patient portal, patients could send messages to their MHC and physician, as well as request refills and referrals. During the observation period, front desk staff assisted patients with portal enrollment. They encouraged them to use it, explained the advantages, and stressed that the doctor wanted all patients to use the portal.

During the group interview, participants mentioned that younger patients, prenatal patients, and parents of pediatric patients were active patient portal users, but its adoption and use had been difficult to achieve among certain patient populations, including the elderly. According to the Medical Home Manager, some patients seemed more inclined to try it after receiving information on its advantages. However, many signed up because they were being told to, but ended up not using it. As highlighted during the interview, others, such as many Haitian elderly patients, had limited computer literacy and would not even try it.

Documents reviewed during the research process, including sample screenshots provided by the health center liaison, showed some of the capabilities of the CHC's EHR, including documentation of different patient encounters like health education and counseling. This system allowed the $\mathrm{CHC}$ to document, assess, and identify risks and 
needs of patients and their family, features needed for population health management. During the group interview, the $\mathrm{COO}$ confirmed that population health management features were currently being used for managed care patients. She explained that the system aggregated data for specific high-risk groups, including high emergency room users, diabetic patients, and behavioral health patients. It also allowed the organization to generate specific reports to facilitate decision-making regarding these groups. She also indicated that, while the system had tools to collect data on social determinants of health, the organization had not yet started collecting this data. According to the $\mathrm{COO}$, they were only documenting patient's living or housing status, since this is a HRSA requirement.

\section{Organizational System and Organizational Culture}

\section{Organizational Structure}

The observation process provided a glance at the size of this organization. As mentioned earlier, this was a multi-story building with different departments and services available throughout all four floors. Several different managers were noticed across departments, which provided a view of the centralization patterns of the organization. However, observations took place at just one of the multiple sites of this health center and not every component of the organizational structure was observable. A review of the CHC's organizational chart provided more information about the size and complexity of this CHC. At the top of the organizational structure was the organization's Board of Directors, which delegated on the Chief Executive Officer (CEO) the responsibility of operationalizing the organization's plans and overseeing daily operations. As seen in the organizational chart, the CEO was also the Chief Medical Officer and Laboratory 
Director. Key management was also constituted by the Chief Operating Officer, Chief Information/Compliance Officer, Chief Medical Informatics Officer, and Chief Financial Officer, each in charge of one or more departments and functions. The COO, for instance, was responsible for Patient Services and the PCMH program. Other key positions included the HIV/AIDS Services executive, Strategic Partnerships and Programs executive, Human Resources executive, and the Grant and Development Director.

The structure of the PCMH program was discussed with the $\mathrm{COO}$ during the group interview. As explained by the $\mathrm{COO}$, the $\mathrm{COO}$, as Patient Services leader, guided the operationalization of the PCMH team, constituted by the Medical Home Manager, physicians, nurses, scribes, medical assistants, medical home coordinators, referral coordinators, and front desk teams. She added that the pharmacy authorization staff was also a key piece of the program's structure and part of the multidisciplinary team. She also pointed out that multidisciplinary teams and the MH Manager collaborated in operationalizing this program.

Observations, the review of the organizational chart, and interviews with key informants revealed the existence of a clearly defined hierarchy and an active involvement of department supervisors, managers, and multidisciplinary teams in decisions regarding care and daily department operations. As mentioned by the CEO during the interview, the health center's leadership placed emphasis on ongoing supervision as a way to ensure their vision is followed. He stressed that, being on top of the staff is a key to the success of this $\mathrm{CHC}$. The $\mathrm{CEO}$ and the $\mathrm{COO}$ made rounds every morning, looking at everything, from lighting to how patient care was delivered. "It's an overall monitoring that you have to do on a daily basis," she added. 


\section{Organizational Culture, Leadership Culture, and Staff Characteristics}

The organization's collaboration, teamwork, and leadership cultures were also examined through observations, the review of materials on the communication of policies, and interviews. Staff members could be seen working in groups, as part of teams, and consulting one another. In addition, the review of the Interdisciplinary Teams Policy documented the leadership's focus on team work to provide care. Daily observations of these teams throughout a week supported the implementation of this policy. Health care team members were observed collaboratively planning, coordinating, and delivering patient care, as well as determining strategies needed to improve patient care processes. In particular, this behavior was observed during the "team huddle", the Medical Home Team meeting, and daily interactions of medical home coordinators and other health care team members. In addition, MHCs were very emphatic during interviews about the importance of teamwork in getting things done, especially when each MHC can be assigned up to five different providers.

Views regarding the health center's "patient-centered" culture were consistent across interviews. It was also evident in observation sessions of staff behaviors, where patients were frequently observed been treated as members of the family and called by their first names on a usual basis. Staff members demonstrated patience and understanding when patients shared different situations and challenges. During all observations, staff were seen treating patients in a very warm manner, reiterating several times that they were there to help them and wanted them to be well. They also seemed highly skilled in addressing patients' concerns and dealing with difficult personalities. 
During observations and in interviews, health care team members showed genuine concern for the patients' well-being and the patient care process. One of the MHCs interviewed shared that the organization made sure that the patient comes first. She added: "the patient is the reason for the organization's existence. Without the patient, we wouldn't be here in the first place. They are the number one priority." According to the $\mathrm{COO}$, the way the organization handled care coordination evidenced their focus on "patient-centeredness". In spite of the "patient centeredness" approach, interviews suggested that the CEO viewed the organization's approach from a decidedly paternalistic perspective. Both of these perspectives were shared with members of the team during "huddles" and Medical Home meetings, as evidenced by reviews of several meeting minutes.

Leadership behaviors were observed in program managers, head nurses, leading physicians, and department champions. According to the Nursing Supervisor in one of the departments observed, one key success factor for the department was the leading provider's vision and role as a leader. "He set a patient care vision for his department and ensures this vision is well understood and followed by his staff." The physician's role as the health care team leader was evident during observations at the "team huddle". The male physician in this case showed his sense of responsibility for guiding the team in the accomplishment of their roles, making sure they followed the PCMH model, but also encouraging them to participate and present their perspectives. This pattern was observed at the corporate level and during Medical Home Team meetings.

During interviews with the corporate level, the Medical Home manager, the Patient Services executive, and the CEO, showed great involvement, engagement, and 
interest in supporting the development and progress of the model. Furthermore, interviews with the $\mathrm{COO}$ and the $\mathrm{MH}$ Manager confirmed the existing level of understanding about the process and activities taking place across the CHC. As evidenced by PCMH policies and communications reviewed, the health center leadership established clear goals, especially regarding patient care, quality, and PCMH performance. These goals were reiterated during the observed "team huddles" and Medical Home Team meetings.

\section{Learning Culture: Performance and Quality Improvement}

According to the $\mathrm{COO}$, the Quality Management team tracks and assesses quality and performance. Documents reviewed, including performance measurement and selfassessment tools, showed that the organization analyzed performance and quality improvement on a quarterly basis to determine progress on multiple measures related to preventive care and chronic disease management, among others. In addition, as observed in an editorial board at the department's conference room, the organization monitored and shared with staff and visitors different accomplishments and quality improvement goals.

Evidence from documents reviewed, including a PCMH self-assessment and the Record Review Workbook (RRWB), supported the organization's overall concern with quality improvement and assessing the implementation of the PCMH model. Samples

provided by the health center liaison for review during the research process showed how the organization examined evidence of PCMH elements in patient records. As observed in the Medical Home Team meeting minutes, the PCMH self-assessment and evidence of PCMH elements in patient records were topics of discussion by the Medical Home team. 
Moreover, as explained by the $\mathrm{COO}$ in the group interview, during the PCMH recognition renewal process, the health center re-assessed its readiness using this assessment tool.

As observed, performance indicators were shared with staff members through internal communications, meetings, and posters on bulletin boards. Team huddles also provided a space to discuss areas and practices that need improvement. In addition, the health center also held monthly Medical Home team meetings, where MHCs discussed concerns and ideas, barriers and facilitators, PCMH updates, new projects, and patient services. Several Medical Home Team meeting minutes were reviewed as part of the research process.

\section{Strategic Partnerships}

Interviews data and documents reviewed revealed the establishment of multiple partnerships with community organizations to support CHC's services. While there were no interactions with partners observed during the site visit period, there were announcements and flyers available for distribution and displayed on bulletin boards about events and educational opportunities in collaboration with community partners. These included a collaboration with the Healthy Start Coalition. The COO also shared information about existing partnerships during one of her interviews. She indicated that the organization had established collaboration with Florida International University's Neighborhood HELP to provide dental services and breast cancer screenings in the community. She also mentioned partnerships with the American Heart Association, American Cancer Society, and Health Foundation of South Florida. Other community strategic partners were identified through the review of health center brochures and 
electronic media. These included: Florida Department of Health, The Children's Trust, Health Choice Network, Health Council of South Florida, Public Health Trust, South Florida AIDS Network, and local pharmaceutical companies.

\section{Medical Home Implementation}

\section{Approach to PCMH implementation}

The different practice elements documented throughout the discussion of emerging themes to this point cover multiple observations regarding the implementation of the PCMH model at this CHC. Given the focus of this study and the way instruments were developed, many of the elements of the model emerged as themes during data analysis. As expressed by the health center's $\mathrm{COO}$, the health organization decided to become a PCMH as a strategy to improve quality and patient outcomes. However, more than just a strategy, observations and interviews showed that the implementation of the PCMH model was a central piece of the organization's functioning.

Observations revealed how each division worked like a medical home itself. Each department had its own health care team with a physician as champion, supported by a head nurse or supervisor and a team of MHCs, RCs, MAs, and other providers needed to address patient needs. Health center leaders interviewed agreed that this structure was a major factor in their success with the implementation of the model. The CHC's PCMH policies corroborated the prominence of this structure.

The review of the above documents provided evidence of the process used by the health center in implementing several key elements of the PCMH model. Policies examined provided a look into the organization's PCMH focus and responsibilities as a medical home, and the patient's role within this model. According to these documents, 
the organization saw the patient as an active agent in the model. The documents also revealed major areas of emphasis in the CHC's PCMH model, including its focus on team-based care, coordination of care, access, quality improvement, and the central role of the EHR in the implementation of the model.

As explained by both the $\mathrm{CEO}$ and $\mathrm{COO}$, the $\mathrm{CHC}$ invested considerable resources to support the implementation of its PCMH model, including significant financial resources, staff, and time. The implementation of this model was very costly for the organization, mainly because of the staff resources dedicated to sustaining the program's structure. MHCs alone were a major expense since they were all LPNs, according to the $\mathrm{COO}$ and $\mathrm{CEO}$. The organization dedicated management resources, to ensure accomplishment of patient care and quality improvement goals.

\section{Challenges and Barriers to Implementation}

Through conversations with key informants at the health center, several organizational challenges and barriers to PCMH implementation of the model were identified. The $\mathrm{COO}$ and $\mathrm{CEO}$ highlighted the resources and processes required to achieve successful care coordination, as established by the model, as two major challenges to PCMH implementation. In addition, multiple complex processes were required for the thousands of encounters and services to be coordinated. According to the CEO, the health center had to invest these significant resources while still providing affordable care for all community members, at the reduced fees agreed by managed care companies. Moreover, it had been difficult to recruit certain positions needed for the implementation of the model, including MHCs, RCs, and operators. In reviewing the 
minutes for the Medical Home Team meetings, personnel shortage was frequently highlighted.

In addition, as evidenced through observations, interviews, policies reviewed, and messages examined, the $\mathrm{CHC}$ invested staff, financial, and time resources in providing self-management support activities, access to an extensive network of health professionals, and ongoing tracking and follow-up. As pointed out by one of the MHCs interviewed, this support had to be provided to the numerous patients affected by chronic diseases. The CEO also highlighted the organization's large investment on a new phone answering system, new patient ID cards with provider and MHCs contact information, new mechanisms for the appointments department, and a network of operators to ensure patient communication with the health center and access to care.

According to health center leaders, complying with PCMH requirements had been a major challenge. As explained by the $\mathrm{COO}$, accrediting agencies provide a list of standards that must not only be followed, but also achieved within a set level or percentage of compliance. She added that some of these standards and compliance levels seemed impossible to achieve at some point. For instance, securing the electronic messaging standard had been very difficult to implement and remained a challenge with a patient population that did not have computer or Internet access or the skills to manage medical technologies.

\section{PCMH Implementation Facilitators}

Several organizational factors emerged as PCMH implementation facilitators for this health center throughout the research and data analysis process. As mentioned earlier, the result of observations, interviews, and documents reviews supported the highly 
structured strategy this $\mathrm{CHC}$ developed to implement the PCMH model. As confirmed by health center leaders during interviews, the health center prioritized the establishment of a PCMH program with the support of a MHCs network as an essential ingredient for the implementation of the model. As observed, CHC leaders were highly involved and engaged in the implementation of the PCMH model. Interviews revealed the willingness to not only invest many financial, staff, and time resources in the process, but also establish organizational mechanisms to make this model a central piece of the organization's functioning. Furthermore, as pointed out by the CEO, leaders ensured ongoing supervision and control over the implementation of the model through daily monitoring. According to the $\mathrm{CEO}$, this made a difference because "the staff knows that there is someone watching and making sure that they are going the extra mile and gives them credit for going that extra mile."

\section{Summary of Key Findings and Organizational Factors Identified}

This section summarizes the salient features of the implementation of the PCMH model observed in Case 1. It also presents a summary of challenges and facilitators to PCMH implementation at this health organization, as revealed by study findings.

\section{Salient Features of CHC1's PCMH Model}

- Service delivery model. The availability of multiple health and patient support services on site facilitated the delivery of patient-centered care, care coordination, and access to care, enhanced by a system of onsite specialists to address multiple health needs, many of whom were key to chronic disease management. 
- Patient-centered culture. Findings showed the CHC leadership's commitment to establishing and sharing a vision for the program and the patient-centered culture. This vision was evident in attitudes and behaviors of staff and leadership, approaches to care coordination, emphasis placed on patient needs, and staff's perspectives about their roles.

- Health care teams. The CHC established a supportive organizational structure for the model that included a fixed medical home team of at least five health professionals in each health center department. A key feature of this structure was the availability of a network of medical home coordinators, responsible for ensuring care coordination, health care planning, monitoring and follow-up, patient education, and self-care support.

- Commitment to "team huddles". Patient-centered care at this CHC seemed to benefit from daily, structured "team huddles" taking place in every department across the organization. CHC leadership monitored compliance with "team huddles".

- Active leadership involvement in PCMH implementation. The health center's leaders had a key role in enforcing PCMH policies and providing continuity to the implementation of practices under the PCMH model, as evidenced by leadership's efforts in following-up and monitoring compliance with PCMH standards.

- Central role of the EHR. The incorporation of the EHR was crucial to patient communication and engagement, follow-up, care coordination processes, monitoring of chronic disease measures, identification of gaps in care, continuity of care, and identification of patient populations at risk, among others. 
- Communication with patients. The health center maintained ongoing communication with patients using different strategies on site and through its website, social networks, and the patient portal.

- Incorporation of chronic disease management to the PCMH strategy. Members of the health care team placed emphasis on ongoing patient monitoring and follow up, education, and self-care support. A major part of this CHC's strategy was the establishment of health care teams entirely focused on chronic care, such as the Diabetes Management department, which incorporated a network of onsite specialists.

- Leadership culture. Top leadership control and oversight and the establishment of a central Medical Home Team guided by the $\mathrm{COO}$ and the Medical Home Manager maintained fidelity to the implementation of the model. CHC leadership also established clear goals regarding patient care, quality, and PCMH performance. The CHC ensured the leadership's vision was disseminated and followed through ongoing supervision.

- Teamwork culture. Teamwork culture was another foundation of PCMH implementation at this $\mathrm{CHC}$, evident at both the corporate and practice levels. Health care team members worked together to collaboratively plan, coordinate, deliver patient care, and determine strategies needed to improve patient care.

- Learning culture and performance improvement. Focusing on improving performance, both in terms of health outcomes and PCMH implementation, the health center established multiple processes for monitoring and analyzing quality 
improvement and PCMH compliance, supported by quality, health care, and Medical Home teams.

- Strategic partnerships. The CHC established multiple partnerships with community organizations not only to support delivery of comprehensive services but also the implementation of the PCMH model.

- PCMH model as a central piece of this organization's functioning. Findings showed that the implementation of the PCMH model was a central piece of this organization's functioning, with each department working as a medical home itself. The $\mathrm{CHC}$ invested in infrastructure and staff resources needed to support this model.

\section{Summary of Challenges and Facilitators to PCMH Implementation}

Table 6 summarizes the major challenges and facilitators to PCMH implementation identified during the research process at $\mathrm{CHC} 1$.

\section{Table 6.}

Summary of Challenges and Facilitators to PCMH Implementation at $\mathrm{CHC} 1$

\begin{tabular}{|l|l|}
\hline Challenges & a. $\begin{array}{l}\text { Multiple resources and complex processes required for the } \\
\text { thousands of encounters and services to be coordinated. } \\
\text { b. Making significant investments while maintaining affordable care } \\
\text { for the community. }\end{array}$ \\
c. Difficulties encountered in recruiting positions needed for the \\
implementation of the model, including MHCs, RCs, and \\
operators. \\
d. Staff shortage in positions required to implement care \\
e. Lardination processes. \\
f. Impact of communications on patients' access to the health center \\
g. Complying with electronic messaging standards given patients' \\
h. Pack of computer and Internet access and technology skills. \\
h. Patients' low compliance with treatment and recommendations. \\
\hline
\end{tabular}




\begin{tabular}{|c|c|}
\hline Facilitators & $\begin{array}{l}\text { a. Availability of multiple onsite health and patient support services. } \\
\text { b. Establishment of a clear vision for the program and patient- } \\
\text { centered culture } \\
\text { c. Establishment of organizational mechanisms and a highly } \\
\text { structured strategy to make this model a central piece of the } \\
\text { organization's functioning. } \\
\text { d. Establishment of health care teams to operationalize PCMH } \\
\text { implementation. } \\
\text { e. Commitment to daily, structured "team huddles" to plan and } \\
\text { coordinate care. } \\
\text { f. Investment in a network of medical home coordinators to sustain } \\
\text { care coordination. } \\
\text { g. Leaders highly involved and engaged in the implementation of the } \\
\text { PCMH model. } \\
\text { h. Significant investments in financial, staff, and time resources } \\
\text { needed. } \\
\text { i. Ongoing leadership supervision and control over the } \\
\text { implementation of the model. } \\
\text { j. Emphasis on continuing learning and performance and quality } \\
\text { improvement. } \\
\text { k. Establishment of strategic partnerships at the community level to } \\
\text { support PCMH implementation. } \\
\text { l. Establishment of health care teams focused on chronic disease } \\
\text { management. Development of strategies to maintain ongoing communication } \\
\text { with patients through multiple means. } \\
\text { Incorporation of health information systems to facilitate } \\
\text { compliance with PCMH standards. }\end{array}$ \\
\hline
\end{tabular}




\section{Chapter IV}

\section{CASE 2}

This chapter presents data analysis and findings from research conducted at the second participating community health center $(\mathrm{CHC} 2)$. As with Case 1, findings from the research process conducted at $\mathrm{CHC} 2$ are presented as an individual case developed after conducting data analysis on field observations, documents, and interviews. The research process was facilitated by the CHC's Executive Director and the director of the observed site. After accepting the invitation to participate in the study, the Executive Director suggested conducting observations and interviews at a delivery site recognized as a PCMH by a national accrediting organization. According to the $\mathrm{CHC}$ leader, while most sites followed the PCMH model, only one had official recognition at the time of the research. The executive designated the Health Information Manager, who was the PCMH leader, as the corporate level informant.

A total of 35 hours of observation were completed at $\mathrm{CHC} 2,30$ of which took place in public areas at the site facility, patient waiting rooms, hallways, and general service areas. The remaining five hours included three hours of observations at the corporate facility and two hours in the community and site location. In addition to observations conducted at the site, 39 documents were carefully reviewed as part of this research process. These are summarized in Table 7. In addition, the CHC's website and social network account posts were reviewed. 


\section{Table 7.}

Documents reviewed as part of the research process at $\mathrm{CHC} 2$

\begin{tabular}{|l|c|l|}
\hline \multicolumn{1}{|c|}{ Type } & Number & \multicolumn{1}{c|}{ Examples } \\
\hline Samples & 8 & $\begin{array}{l}\text { Worksheets (PCMH Record Review) } \\
\text { PCMH self-assessments } \\
\text { Patient flow at PCMH site } \\
\text { Sample EHR screenshots (unidentified) } \\
\text { Sample list of community resources }\end{array}$ \\
\hline Forms and tools & 6 & $\begin{array}{l}\text { Pre-visit patient questionnaire } \\
\text { Pediatric to adult care transition form } \\
\text { Daily tracking of community referrals } \\
\text { "Team huddle" documentation form } \\
\text { Health care plan goals form } \\
\text { PCMH site monitoring tool }\end{array}$ \\
\hline $\begin{array}{l}\text { Patient } \\
\text { communications }\end{array}$ & 6 & $\begin{array}{l}\text { Notice about the PCMH model } \\
\text { Educational brochures and flyers sampled at site }\end{array}$ \\
\hline $\begin{array}{l}\text { Health Center } \\
\text { information }\end{array}$ & 2 & $\begin{array}{l}\text { Health center brochure } \\
\text { Organizational chart }\end{array}$ \\
\hline $\begin{array}{l}\text { Reports and work } \\
\text { plans }\end{array}$ & 7 & $\begin{array}{l}\text { CHC progress reports } \\
\text { PCMH progress reports } \\
\text { PCMH work plan } \\
\text { Patient satisfaction survey report }\end{array}$ \\
\hline Health Center policies & 7 & $\begin{array}{l}\text { Access to clinical advice } \\
\text { Phone triage } \\
\text { Patient support through the Outreach and } \\
\text { Enrollment Program } \\
\text { Walk-in visits and same day appointments } \\
\text { Hospital transfers } \\
\text { Sharing clinical information with hospital } \\
\text { Educating patients about PCMH } \\
\text { Policy on communication with English speakers }\end{array}$ \\
\hline Meeting documents & 3 & \begin{tabular}{l} 
PCMH team meetings (3 meetings) \\
\hline
\end{tabular} \\
\hline
\end{tabular}

The final data collection process included a total of six individual semi-structured interviews with health center key informants: the observed site's administrator, health educator, nutritionist, and nursing supervisor, the health center's Health Information 
Manger (PCMH leader) and the quality manager. In total, nearly 6 hours (355 minutes) of interviews were conducted at $\mathrm{CHC} 2$. Interview duration ranged from 15 minutes to 2 hours. Since this health center was located in Puerto Rico, the organization's staff spoke Spanish. Interviews were conducted in Spanish, transcribed verbatim and then translated to English for the purpose of data analysis and reporting. The resulting case was shared with the health center to validate findings. Table 8 includes the list of questions used as guidelines during interviews with key informants. Each category of questions is aligned with the themes that emerged during data analysis and associated emerging terms.

Table 8.

Case 2 Interview Guiding Questions and Emerging Themes

\begin{tabular}{|c|c|c|c|}
\hline Topic & Guiding Questions & $\begin{array}{c}\text { Emerging } \\
\text { Themes }\end{array}$ & Associated Terms \\
\hline $\begin{array}{l}\text { Organizational } \\
\text { culture }\end{array}$ & $\begin{array}{l}\text { 1. Tell me about your } \\
\text { organization. } \\
\text { 2. How would you } \\
\text { describe it? } \\
\text { 3. How would you define } \\
\text { the people working at } \\
\text { the organization? }\end{array}$ & $\begin{array}{l}\text { Approach to } \\
\text { patient- } \\
\text { centeredness } \\
\text { Organizational } \\
\text { culture, } \\
\text { leadership } \\
\text { culture, and } \\
\text { staff } \\
\text { characteristics }\end{array}$ & $\begin{array}{l}\text { Leadership } \\
\text { Patient-centered } \\
\text { culture } \\
\text { Patient service } \\
\text { culture }\end{array}$ \\
\hline $\begin{array}{l}\text { Patient } \\
\text { Centered } \\
\text { approach }\end{array}$ & $\begin{array}{l}\text { 4. How would you define } \\
\text { "patient-centered"? } \\
\text { 5. What makes this a } \\
\text { patient-centered } \\
\text { organization? } \\
\text { 6. What do you do to } \\
\text { maintain the "patient- } \\
\text { centeredness" of the } \\
\text { health center? } \\
\text { 7. Does this focus you're } \\
\text { describing fit the } \\
\text { characteristics or }\end{array}$ & $\begin{array}{l}\text { Need-based } \\
\text { care } \\
\text { Comprehensive } \\
\text { services } \\
\text { Organizational } \\
\text { culture, staff } \\
\text { characteristics } \\
\text { Patient support } \\
\text { Patient/family } \\
\text { participation } \\
\text { Approach to } \\
\text { patient- } \\
\text { centeredness }\end{array}$ & $\begin{array}{l}\text { Patient needs } \\
\text { Assessment of } \\
\text { needs } \\
\text { Patient/family } \\
\text { participation } \\
\text { Patient Service } \\
\text { culture } \\
\text { Learn about patient } \\
\text { Relationship with } \\
\text { patient } \\
\text { Assessment of } \\
\text { family environment } \\
\text { Comprehensive care }\end{array}$ \\
\hline
\end{tabular}




\begin{tabular}{|c|c|c|c|}
\hline & $\begin{array}{l}\text { essence of your } \\
\text { organization? How? }\end{array}$ & & $\begin{array}{l}\text { Vulnerable } \\
\text { populations } \\
\text { Patient experience, } \\
\text { satisfaction }\end{array}$ \\
\hline $\begin{array}{l}\text { PCMH and } \\
\text { Chronic } \\
\text { Disease } \\
\text { Management }\end{array}$ & $\begin{array}{l}\text { 8. How does this "patient- } \\
\text { centered" approach you } \\
\text { are describing relate to } \\
\text { your work with chronic } \\
\text { disease management? } \\
\text { 9. How do you use that } \\
\text { "patient-centered" } \\
\text { strategy to support } \\
\text { chronic disease } \\
\text { management? } \\
\text { (Examples) } \\
\text { 10. Describe what your } \\
\text { organization does to } \\
\text { support chronic disease } \\
\text { management } \\
\text { 11. What is and/or how do } \\
\text { you see your role in this } \\
\text { process? }\end{array}$ & $\begin{array}{l}\text { Team-based } \\
\text { care } \\
\text { Care } \\
\text { coordination } \\
\text { Planned care } \\
\text { Self-care } \\
\text { support } \\
\text { Patient } \\
\text { education } \\
\text { Patient } \\
\text { communication } \\
\text { Patient } \\
\text { participation } \\
\text { Quality } \\
\text { improvement } \\
\text { Chronic } \\
\text { disease } \\
\text { management } \\
\text { Use of } \\
\text { technology } \\
\text { Patient Support }\end{array}$ & $\begin{array}{l}\text { Chronic diseases } \\
\text { Overweight } \\
\text { Diabetes } \\
\text { Hypertension } \\
\text { Health care team } \\
\text { Doctor, provider } \\
\text { Nutritionist } \\
\text { Patient evaluation } \\
\text { Follow-up } \\
\text { Health educator } \\
\text { Social worker } \\
\text { Nurse } \\
\text { Referrals } \\
\text { Coordination } \\
\text { Collaboration } \\
\text { Meetings } \\
\text { Education } \\
\text { Patient Service } \\
\text { Behavioral change } \\
\text { EHR documentation } \\
\text { Physical activity } \\
\text { Educational events } \\
\text { Home visits } \\
\text { Self-care support } \\
\text { Family participation } \\
\text { Performance } \\
\text { measures } \\
\text { Patient goals } \\
\text { Patient compliance } \\
\text { Adherence to } \\
\text { treatment }\end{array}$ \\
\hline $\begin{array}{l}\text { PMCH } \\
\text { Implementation }\end{array}$ & $\begin{array}{l}\text { 12. Tell me about your } \\
\text { experience } \\
\text { implementing the } \\
\text { Patient-Centered } \\
\text { Medical Home model } \\
\text { 13. What were some of the } \\
\text { key changes you had to } \\
\text { go through? }\end{array}$ & $\begin{array}{l}\text { Approach to } \\
\text { implementation } \\
\text { Comprehensive } \\
\text { care } \\
\text { Patient support } \\
\text { Culturally and } \\
\text { linguistically } \\
\text { appropriate } \\
\text { services }\end{array}$ & $\begin{array}{l}\text { Health care team } \\
\text { Provider } \\
\text { Nutritionist } \\
\text { Health Educator } \\
\text { Social Worker } \\
\text { Nurse } \\
\text { Coordination } \\
\text { Follow-up } \\
\text { Referrals } \\
\text { Collaboration }\end{array}$ \\
\hline
\end{tabular}




\begin{tabular}{|c|c|c|c|}
\hline & $\begin{array}{l}\text { 14. The model has several } \\
\text { components. Can you } \\
\text { tell me about how your } \\
\text { organization covers } \\
\text { these components? }\end{array}$ & $\begin{array}{l}\text { Patient } \\
\text { participation } \\
\text { Access to care } \\
\text { Team-based } \\
\text { care } \\
\text { Care } \\
\text { coordination } \\
\text { Planned care } \\
\text { Use of } \\
\text { technology } \\
\text { Population } \\
\text { health } \\
\text { management } \\
\text { Organizational } \\
\text { structure } \\
\text { Organizational } \\
\text { culture } \\
\text { Leadership } \\
\text { culture } \\
\text { Performance } \\
\text { and quality } \\
\text { improvement } \\
\text { Strategic } \\
\text { partnerships } \\
\text { Approach to } \\
\text { patient- } \\
\text { centeredness }\end{array}$ & $\begin{array}{l}\text { Meetings } \\
\text { Communication } \\
\text { Interdisciplinary } \\
\text { Patient Service } \\
\text { Support services } \\
\text { Comprehensive } \\
\text { services } \\
\text { Specialists } \\
\text { Health care plan } \\
\text { Planned visits } \\
\text { Pre-visit } \\
\text { Team huddles } \\
\text { Site level } \\
\text { implementation } \\
\text { Home visits } \\
\text { Community events } \\
\text { Partnerships } \\
\text { Outpatient } \\
\text { Department } \\
\text { Access to care } \\
\text { Walk-in/Same Day } \\
\text { Tracking } \\
\text { PCMH leader } \\
\text { PCMH assessment } \\
\text { Quality } \\
\text { improvement } \\
\text { PDSA cycle } \\
\text { Planning } \\
\text { Training } \\
\text { Policies } \\
\text { Patient-centered } \\
\text { culture } \\
\text { PCMH recognition } \\
\text { Standards/ } \\
\text { guidelines/ } \\
\text { requirements } \\
\text { Transformation } \\
\end{array}$ \\
\hline Facilitators & $\begin{array}{l}\text { 15. Let's talk about the } \\
\text { things or factors you } \\
\text { believe contributed to } \\
\text { the implementation of } \\
\text { the PCMH model } \\
\text { (anything that facilitated } \\
\text { the process). Which }\end{array}$ & $\begin{array}{l}\text { Patient } \\
\text { communication } \\
\text { and education } \\
\text { Comprehensive } \\
\text { care } \\
\text { Patient support } \\
\text { Patient } \\
\text { participation in }\end{array}$ & $\begin{array}{l}\text { EHR } \\
\text { Leadership support } \\
\text { Leadership } \\
\text { commitment } \\
\text { Patient } \\
\text { communication } \\
\text { Coordination }\end{array}$ \\
\hline
\end{tabular}




\begin{tabular}{|c|c|c|c|}
\hline & $\begin{array}{l}\text { organizational attributes } \\
\text { do you think contributed } \\
\text { to this process? } \\
\text { 16. Tell me about your roles } \\
\text { in this process. } \\
\text { 17. What can you say about } \\
\text { the role of the health } \\
\text { center's leadership in } \\
\text { this process? }\end{array}$ & $\begin{array}{l}\text { the care } \\
\text { process } \\
\text { Access to care } \\
\text { Team-based } \\
\text { care } \\
\text { Care } \\
\text { coordination } \\
\text { Use of } \\
\text { technology to } \\
\text { facilitate } \\
\text { patient care } \\
\text { Organizational } \\
\text { and leadership } \\
\text { culture } \\
\text { Performance } \\
\text { and quality } \\
\text { improvement } \\
\text { Strategic } \\
\text { partnerships } \\
\text { Facilitators }\end{array}$ & $\begin{array}{l}\text { Interprofessional } \\
\text { communication } \\
\text { Patient-centered } \\
\text { vision } \\
\text { Resources } \\
\text { Outpatient } \\
\text { Department } \\
\text { Comprehensive } \\
\text { services } \\
\text { Nurse leader } \\
\text { Nursing staff } \\
\text { PCMH leader } \\
\text { PCMH committees } \\
\text { Leadership } \\
\text { involvement/support } \\
\text { Follow up } \\
\text { Policies } \\
\text { CHC model }\end{array}$ \\
\hline Barriers & $\begin{array}{l}\text { 18. Now, let's think about } \\
\text { barriers or obstacles } \\
\text { encountered along the } \\
\text { way. Which factors or } \\
\text { issues do you believe } \\
\text { make it difficult to } \\
\text { implement the } \\
\text { components of this } \\
\text { model? }\end{array}$ & $\begin{array}{l}\text { Barriers to } \\
\text { implementation } \\
\text { Patient } \\
\text { communication } \\
\text { Access to care } \\
\text { Team-based } \\
\text { care } \\
\text { Care } \\
\text { coordination } \\
\text { Patient support } \\
\text { Approach to } \\
\text { PCMH } \\
\text { Use of } \\
\text { technology }\end{array}$ & $\begin{array}{l}\text { Patient flow } \\
\text { Physical } \\
\text { infrastructure } \\
\text { Appointment } \\
\text { policies } \\
\text { Staff shortage } \\
\text { Shared staff } \\
\text { Administrative } \\
\text { changes } \\
\text { Site level } \\
\text { implementation } \\
\text { EHR capacity } \\
\text { Patient non- } \\
\text { compliance } \\
\text { Nurse workload } \\
\text { Communication } \\
\text { system } \\
\text { Patient access } \\
\text { Behavioral health } \\
\text { Staff commitment }\end{array}$ \\
\hline
\end{tabular}

In what follows, data are presented according to the themes that naturally emerged from observations and interview data in response to the questions guiding the 
study. Rather than grouping data according to the structured format of the questions, the choice was made to allow the data to flow as it naturally emerged from the dynamic exchanges that occurred while interviewing or during observations. Data analysis resulted in 19 themes that emerged from the combined three data sources. These themes aligned with the focus of this study and served as guidelines to develop both cases. This chapter begins with an overview of $\mathrm{CHC} 2$, followed with a description of the site, a summary of emerging themes, as identified from the data analysis and an integrated discussion of findings by theme. The last section summarizes the key findings and major challenges and facilitators identified at this health center.

\section{Community Health Center 2 (CHC2) Overview}

CHC2 is one of the 20 HRSA-funded CHCs serving the population of the US Commonwealth of Puerto Rico. In 2017, this health center, founded nearly 45 years ago, served almost 40,000 patients across seven (7) delivery sites in multiple rural municipalities of Puerto Rico; over 5,000 patients were seen at the observed site. Nearly all patients served by this organization (over 98\%) are low-income and $90 \%$ live at or below the federal poverty level (HRSA, 2018c). Over three-fourths are Medicaid/CHIP beneficiaries, compared to only one-fifth of the population in Case 1; over one-third of patients in Case 1 were uninsured. On the other hand, similar to Case 1, population served at CHC2 is largely affected by chronic diseases. Over 30\% have a hypertension diagnosis and more than $12 \%$ have diabetes (HRSA, 2018c).

One key characteristic of CHCs operating in Puerto Rico is the homogeneity of its population. Close to $99 \%$ of patients served by CHC2 are Hispanic/Latino (HRSA, 2018c). The majority of these patients are Puerto Rican. This level of sameness observed 
at this health center contrasts with the reality of other $\mathrm{CHCs}$ across the nation, including Case 1 . Both patients and providers observed at Case 1 were culturally diverse. Even when close to $70 \%$ of patients served by $\mathrm{CHC} 1$ are Hispanic/Latino, their Hispanic origins are also diverse. Additionally, in Case 2, cultural homogeneity is also observed among staff and providers.

\section{Site Description}

As shown above, 30 hours of observation at this $\mathrm{CHC}$ took place at one of its service delivery sites, the only site recognized as PCMH at the time of the study. The observed site was a recently renovated facility in a rural town in the central northern region of Puerto Rico with an estimated population of almost 35,000. Even though the site was located in a mountainous region with very steep roads, it was easily accessible by car from one major highway. It could be more challenging for municipality residents without their own means of transportation, as there was only one collective transportation route available.

The first visit took place a Friday morning, around 8:30 am, just five months after Hurricane Maria. It was easy to find the site from this very first visit. This was a brightlypainted, modern, medium-size, three-story facility of nearly 30,000 square feet of office space that still looked brand-new. There were many other commercial buildings in the area, including fast food restaurants, pharmacies, local stores, and a non-affiliated hospital facility right beside the site. At first, it was somewhat difficult to know where to go, as there was no general reception area at the entrance. Yet, at the end of the first-floor hallway, there was a large directory with the departments and service areas available. The 
facility was easy to navigate, as most service areas were clearly identified. All building signs were in Spanish.

Each functional and service area was clearly delimited. The first floor of the building had multiple departments: Outpatient Department (OPD), Laboratory, Imaging, Mammography, Referrals, Pharmacy, and Information Management. Except for OPD, all provider offices were on the third floor: Pediatrics, Gynecology, Immunization, Social Work, Nutrition, Health Education, and Primary Care. There were several waiting rooms throughout service areas and a larger waiting area for Primary Care and allied health professions. Most waiting areas were crowded during most of the six days of site observation. There were patients from all age groups: children, young adults, middleaged, and elderly. They were all Hispanic. This population profile differs from the one observed in Case 1, where most of the patients were adults, mainly middle-aged, from various ethnic backgrounds.

\section{Emerging Themes}

Following the analytical methods employed in Case 1, Nvivo and line-by-line analysis were used in examining emerging themes for Case 2. After transcribing, translating, documenting, and organizing data yielded by the three methods, resulting texts were imported to Nvivo, one source at a time. To validate and confirm themes, a line-by-line text analysis was conducted. Appendix $\mathrm{H}$ details the emerging themes identified by this method, paired with a list of related key terms. The following sections discuss emerging themes, organized into six major categories, as shown in Table 9. 
Table 9.

Categorized emerging themes for Case 2

\begin{tabular}{|l|l|}
\hline \multicolumn{1}{|c|}{ Categories } & \multicolumn{1}{c|}{ Emerging themes } \\
\hline $\begin{array}{l}\text { 1. Patient-centered, } \\
\text { need-based care }\end{array}$ & $\begin{array}{l}\text { Comprehensive, integrated care } \\
\text { Patient support } \\
\text { Culturally and linguistically appropriate services } \\
\text { Patient/family participation in the care process } \\
\text { Access to care }\end{array}$ \\
\hline $\begin{array}{l}\text { 2. Coordination and } \\
\text { integration of care }\end{array}$ & $\begin{array}{l}\text { Team-based care } \\
\text { Care coordination practices } \\
\text { Planned care }\end{array}$ \\
\hline $\begin{array}{l}\text { 3. Education and self- } \\
\text { care support }\end{array}$ & $\begin{array}{l}\text { Patient Communication and Education } \\
\text { Chronic disease management and self-management/self- } \\
\text { care support }\end{array}$ \\
\hline $\begin{array}{l}\text { 4. Organizational } \\
\text { system and culture }\end{array}$ & $\begin{array}{l}\text { Organizational structure } \\
\text { Organizational and leadership culture } \\
\text { Learning climate, performance and quality improvement } \\
\text { Strategic partnerships }\end{array}$ \\
\hline $\begin{array}{l}\text { 5. Technology } \\
\text { integration }\end{array}$ & $\begin{array}{l}\text { Technology use to facilitate patient care and manage } \\
\text { population health }\end{array}$ \\
\hline $\begin{array}{l}\text { 6. Medical Home } \\
\text { implementation }\end{array}$ & $\begin{array}{l}\text { Approach to patient-centeredness } \\
\text { Approach to PCMH implementation } \\
\text { Challenges and barriers to implementation } \\
\text { Implementation facilitators }\end{array}$ \\
\hline
\end{tabular}

\section{Patient-Centered, Need-Based Care}

\section{Comprehensive and Integrated Care}

The selected site, one of seven making up the health center, provided a first-hand opportunity to observe the multiple services offered. At this delivery site, services were provided under one roof, allowing patients to move throughout the facility as needed. While at the site, several patients were observed with multiple service appointments in one day, a scenario also seen in Case 1. According to the nursing supervisor, having diverse onsite services to address multiple health care needs was attractive to community 
residents, especially because of shortages of health services and health professionals in the communities served. Table 10 organizes services offered across the health center's multiple sites.

\section{Table 10.}

Health services provided by $\mathrm{CHC} 2$

\begin{tabular}{|l|l|}
\hline $\begin{array}{l}\text { Preventive and } \\
\text { Primary Care }\end{array}$ & $\begin{array}{l}\text { Preventive screenings, General Medicine, Family Medicine, } \\
\text { Internal Medicine, Pediatrics, chronic disease management, } \\
\text { Obstetrics/Gynecology, prenatal care, laboratory, radiology, } \\
\text { sonography, pharmacy, immunization, emergency care }\end{array}$ \\
\hline $\begin{array}{l}\text { Behavioral } \\
\text { Health }\end{array}$ & Mental health counseling, clinical social work, tobacco cessation \\
\hline Oral Health & Dental services (preventive and basic) \\
\hline Specialty Care & Cardiology, Endocrinology, referrals to specialists \\
\hline HIV/AIDS & $\begin{array}{l}\text { Prevention, testing, counseling, outreach services, case } \\
\text { management, treatment }\end{array}$ \\
\hline $\begin{array}{l}\text { Enabling/ } \\
\text { Patient Support }\end{array}$ & $\begin{array}{l}\text { Health education, nutrition, case management, family planning, } \\
\text { outreach and enrollment, transportation, patient transport/transfer, } \\
\text { prescription delivery }\end{array}$ \\
\hline $\begin{array}{l}\text { Special } \\
\text { programs }\end{array}$ & $\begin{array}{l}\text { Mobile unit, health programs for agricultural workers, home care, } \\
\text { hospice, Zika prevention and management, Healthy Families } \\
\text { (support program for young prenatal patients under 21) }\end{array}$ \\
\hline
\end{tabular}

Documents reviewed, including organizational reports and brochures, the health center's website, and interviews corroborated this CHC's system of patient referral to other sites when a service was not available at one of the sites. For instance, not every site had mammography services, but the services were available within the CHC's network and patients were referred internally. The main delivery site, located 25 to 30 
minutes by car from the observed site, included specialists, transportation services, and emergency care, among others.

\section{Patient Support and Enabling Services}

As observed in announcements posted throughout the waiting rooms and hallways at the site and organizational reports reviewed, the $\mathrm{CHC}$ provided options to minimize barriers to care and help patients address other life challenges. For example, as observed in Case 1, there were announcements indicating the availability of a sliding fee discount, medication discounts, and the organization's policy to serve all patients regardless of their capacity to pay for services. Additionally, the Outreach and Enrollment (O\&E) Program, as noticed in multiple organizational reports, the CHC's website, and published policies, helped patients and families obtain health coverage either through Medicaid, CHIP, other medical assistance programs in Puerto Rico, or the Sliding Fee Discount program. Health center reports and information available through the website also documented existing programs to support vulnerable populations, such as agricultural workers and prenatal patients under 21.

\section{Culturally and Linguistically Appropriate Services}

All patients seen during the 30 hours of onsite observation were Hispanic. This is not surprising since the center was not only located in Puerto Rico, but also in a town outside of San Juan's large and cosmopolitan region. As mentioned earlier in this chapter, this patient profile not only differed from the first case, but also gave this $\mathrm{CHC}$ and other CHCs in Puerto Rico a level of homogeneity and ethnic uniqueness not found anywhere else in US mainland. The easy-going communication style patients used when talking to 
one another in waiting rooms was immediately noticeable and entered in the observation journal. The lively conversations and exchanges observed at this site contrasted with observations recorded at the South Florida site (Case 1), where patients were unlikely to engage in conversations while waiting. At the Puerto Rico site, patients behaved as friends, openly and spontaneously sharing different health problems and remedies.

Staff members at this site also used a familiar and colloquial communication style with patients. The health educator and nutritionist, for example, relied on local phrases to discuss health problems in a humorous tone. According to the health educator, nutritionist, and nursing supervisor, their informal tone was an important part of their interactions with patients. Being a culturally homogeneous organization, this might be easier to achieve than at a highly diverse organization such as Case 1. Similarly, education materials were simple and used everyday language, particularly those developed by the organization locally.

In reviewing reports prepared by the $\mathrm{CHC}$ during the $\mathrm{PCMH}$ re-accreditation process, the organization stated that patient documents were assessed with reference to the communication needs of the population served, including language needs of English speakers. According to the CHC's policies, patients or visitors identified as English speakers were paired with a designated bilingual staff. While most announcements displayed at the site were available in Spanish, signs indicating that the Center served patients regardless of their payment capacity, and those announcing sliding fee discounts, were available in both Spanish and English. When the website was examined, contents were available in both languages. Yet, all of the educational materials displayed and sampled at the site were available only in Spanish. The latter might be a practical 
approach, considering the rare occurrence of English-speaking patients at this site. As discussed in Chapter 3, given patient diversity at the first $\mathrm{CHC}$, the organization was attentive in ensuring availability of communications in the three main languages spoken by patients.

\section{Patient and Family Participation in the Care Process}

As seen during the observation period, one of the CHC's main strategies to encourage patient participation was group education. Throughout the six-day site visit, patients were seen participating in educational sessions on breastfeeding, heart health, and mental health. While this practice was not directly observed at the first health center, group education was also mentioned as a major self-management support strategy for this organization. In addition, according to the health educator, the nursing supervisor, and the PCMH leader at the Puerto Rico site, the CHC regularly organized community events for patients, community members, and their families.

Furthermore, as noted when reviewing documents, the organization had a policy on how to involve patients and family members in quality initiatives, which provided opportunities for patient feedback through suggestions boxes and surveys, and during community meetings. The health center's PCMH brochure included a list of patient roles and responsibilities. The patient was expected to stay actively involved in the process of care, as shared by staff members during interviews. This expectation was also frequently shared by staff at CHC1.

Despite the Center's insistence on family participation, this was not evident during the observation period. Besides children accompanied by their parents, most of the patients observed during the 30 hours of onsite observations seemed to be there by 
themselves, a scenario shared by Case 1. Only two elderly patients were accompanied by younger people who appeared to be their children. During an interview with the nutritionist, she stated that family participation depended on the patient's ability to understand his/her condition and the readiness to receive education. According to the health educator, she encouraged patients to bring a family member during the educational process, especially patients with chronic diseases not able to understand the information provided. The health educator explained: "If you have high blood sugar levels, unfortunately, it will not allow learning, so I always try to have a family member present." In contrast, the nursing supervisor, indicated that, unless the patient has a disability, she did not require the presence of a family member.

\section{Access to Care}

Findings from field observations, document analysis, and interviews revealed multiple efforts by this $\mathrm{CHC}$ to enhance access to care across its delivery sites, such as providing services during extended hours. The observed site provided services Monday through Friday, from 7:30 am to 9:00 pm, and Saturdays until 6:00 pm. The pharmacy was available until 7:00 pm on weekdays. Immunization services could be accessed at six CHC sites from 8:00 am to 4:00 pm. According to a PCMH self-assessment and other organizational documents, the $\mathrm{CHC}$ also provided $24 / 7$ access to clinical advice by phone and through the main site's emergency room.

Documents reviewed, including health center policies and PCMH work plans, showed the availability of walk-in/same-day opportunities for patients. The CHC's Walkin/Same Day Policy established that the organization must have at least three appointment slots in the morning and three in the afternoon for walk-in/same-day patients 
per health care provider. According to staff members interviewed, a successful strategy in facilitating walk-in/same-day appointments was the establishment of an Outpatient Department (OPD) exclusively to attend walk-in and same-day patients, since it had helped balance patient load in the regularly scheduled clinics. This documented formal approach to serve walk-in or same day patients was absent in Case 1, where patients indicated long waits, precisely because of the number of patients who came in without scheduled appointments.

As reports stated, on average the OPD saw 25 patients daily, but the OPD nurse could triage up to 35 walk-in/same-day patients a day. During the observation period, the small 15-seat OPD waiting room remained crowded most of the time. Despite procedures in place, same-day patients appeared to the observer to experience long waiting time to see the provider. At the third-floor waiting room, several patients shared that their waiting times could extend up to four hours. Patient wait time during the observation period was on average 1 to 2 hours; the patient cycle could last from $1 \frac{1}{2}$ to 3 hours, including wait time.

\section{Coordination and Integration of Care}

\section{Team-Based Care}

According to several reports, the organization's health care team consisted of the physician, nurse, health educator, nutritionist, social worker, and patient service officers. Yet, staff perceptions of the health care team's composition varied among those interviewed. The nurse supervisor, for example, indicated that the health care team mainly consisted of the physician, nurse, and a patient service officer. On the other hand, the health educator and nutritionist were emphatic about the role of allied health 
professionals as part of the team. One of the several PCMH reports examined highlighted the CHC's investment in health educators, nutritionists, and social workers to support the health care team. This variability in the conceptualization of the health care team at this second $\mathrm{CHC}$ contrasts with first CHC's emphasis on a fixed PCMH structure followed consistently across the organization.

Upon further reviewing additional reports and interviews, the researcher learned that some health professionals at $\mathrm{CHC} 2$ were currently shared between sites. For instance, at the visited site, the health educator was available three days per week and the psychologist only once weekly. According to an interview with the nursing supervisor, areas such as Patient Service were also short-staffed. While staff-sharing was not observed at the site level in the first case, several areas were also short-staffed and some team members were in charge of multiple departments.

Despite staff insistence that the site had a multidisciplinary team in place, team collaborations were not evident during the 30 hours of observations at the site. Observations indicating this practice were limited to the nursing supervisor seen speaking several times on the phone with other nurses and once with a patient where it was overheard that she had discussed her case with the physician. On the other hand, the dyadic partnership between the health educator and the nutritionist was clear during educational sessions and became a topic for discussion during interviews. According to interviewed allied health professionals, they consulted the doctor throughout the intervention and kept him regularly informed about the patient's progress; however, they mainly collaborated with each other, frequently working jointly on different patient cases. 
When asked about participation in "team huddles" or team meetings, both health professionals indicated that presently they were not meeting with the entire health care team to discuss and analyze a patient's situation. However, they both expressed that they maintained regular communication with the physician and other health professionals. Similarly to "team huddles" described for Case 1 and as stated in a PCMH progress report, the physician and nurse at the observed site met daily to validate scheduled patients, examine records, and discuss triage and calls to patients. However, during the interview with the designated PCMH leader, she explained that "team huddles" were not taking place with the same rigor as before. According to this staff member, despite regular communication between the physician and nurse, these meetings did not occur systematically and are no longer documented. This finding contrasts with observations for Case 1, where "team huddles" were a key feature of the PCMH model and teams followed a standardized process across the organization.

\section{Care Coordination}

During the observation period, patient service officers were frequently seen scheduling appointments at the site, coordinating appointments on the phone, or calling patients to remind them of appointments, similar to medical home coordinators observed at $\mathrm{CHC} 1$. According to interviewees, patient service officers registered and checked patients out, served as liaisons between patients and providers, and coordinated internal services. The nursing supervisor explained that one patient service officer was designated to help patients identify external providers and support with insurance authorizations. Contrary to what was observed in Case 1, patients were responsible for coordinating 
appointments with external providers themselves. Yet, she added that nurses tracked referrals created by physicians in the system.

The health educator, nutritionist, and nursing supervisor explained that referrals for services at either the same site or other sites were managed internally. The nutritionist clarified that there was no need for a formal provider referral for most of these services, except for nutrition, because the health insurance plan required it. Furthermore, if health professionals wanted to schedule a team appointment, they could coordinate the appointment themselves.

According to the nursing supervisor, many of the care coordination responsibilities were assigned to nurses, "on top of the many roles they already have." She continued,

"Many times, I have 400 referrals. We are limited because the nurse has to do everything: interview patients, be on top of the patient's education, complete the PHQ-9, take vitals, document the history of a new patient, and complete the chief complaint."

She added that nurses also followed-up with patients with altered labs. As documented by reports, they also mailed reminders for preventive screenings and other services needed. The combined care coordination role of nurses and patient service officers at CHC2 seemed equivalent to the role of medical home coordinators at $\mathrm{CHC} 1$, who coordinated the entire patient care process, in collaboration with referral coordinators.

\section{Planned Care}

Observations related to the care planning process were limited during the site visit due to the restricted access to patient care areas and absence of "team huddles". However, 
during the interview with the nursing supervisor, opportunities became available to observe the health professional interacting with other providers on the phone. These provided insights about care planning functions at this site. As observed, the nursing supervisor, who was also in charge of the HIV/AIDS program, pre-planned patient visits and prepared patient care plans in collaboration with the physician and the social worker. According to a PCMH progress report, nurses reviewed patient records two days prior to the appointment to identify any preventive procedures pending and prepare a plan for the visit. In interviews, the health educator and nutritionist indicated that they also preplanned scheduled visits. The health educator added that she met briefly with the assigned provider at the start of each week. As she explained, the nurse usually identified cases that needed health education reinforcement. Both pre-planned care and health education were also key aspects of the work of medical home coordinators at CHC1. Information documented in the PCMH work plan showed that the health center designed a standardized health care planning tool for providers across all sites. As seen in sample reports shared by the PCMH leader, providers were expected to develop goals for chronic disease patients and document barriers to accomplish these goals. As observed in the health care planning tool, these goals were developed in collaboration with the patient. According to a PCMH progress report, regardless of PCMH recognition, all delivery sites followed this practice. The PCMH leader explained that the $\mathrm{CHC}$ had always prioritized the development of a health care plan, adding that this was an important part of the Health Disparities Collaborative. 


\section{Education and Self-Care Support}

\section{Patient Communication and Education}

There were multiple evidences of communication and education efforts during the observation period at the PCMH-recognized site. From wall and window displays, editorial boards, and print materials to multiple group education sessions, the observed site seemed to maintain active communication with its patients and an ongoing focus on education. There were announcements on variety of topics, including patients' rights and responsibilities, sliding fee discounts, and health issues such as zika, dengue, and healthy lifestyles. Similar to Case 1, during the observation period, no patients were seen stopping to read these announcements and educational materials. However, patient participation was noted during several observed educational sessions. Throughout the six days of observation at this site, there were three group education sessions: one on breastfeeding, one on heart health, and one on mental health.

According to the health center Education Policy, and confirmed by the health educator, educational sessions and materials were standardized across sites, a practice shared as well by $\mathrm{CHC} 1$. In the case of $\mathrm{CHC} 2$, as explained by the health educator, the Puerto Rico Health Insurance Administration required the $\mathrm{CHC}$ to conduct at least 20 monthly educational talks and, as part of the PCMH recognition, the accrediting agency required educational events at least three times weekly. She stressed that these activities were documented in the record to facilitate tracking of patient participation in educational sessions. 


\section{Chronic Disease Management and Self-Management/Self-Care Support}

Many of the patients observed in the general waiting room seemed to be affected by chronic diseases. While speaking to one another, several mentioned having conditions such as diabetes, hypertension, and asthma. The nutritionist validated that the majority of her patients had chronic conditions. Documents reviewed discussed the incorporation of patient lifestyle goals into the health center's care plan as part of efforts to manage chronic diseases. In addition, the CHC's health care goals form prioritized goals related to self-care, medication adherence, glucose and blood pressure monitoring, stress management, and social support.

Several reports and self-assessments reviewed supported the health center provision of access to self-management support and individual and group education, a strategy observed multiple times during the observation period, as discussed earlier. In addition, according to the PCMH leader, the $\mathrm{CHC}$ developed an integrated chronic disease management effort for a selected group of patients that earned the organization a recognition. While this educational approach to self-management support was also observed in the first $\mathrm{CHC}$, it is worth noting that a major part of $\mathrm{CHC1}$ 's strategy was the establishment of health care teams entirely focused on chronic disease management, such as the Diabetes Management department, which incorporated a network of onsite specialists deemed necessary to maintain diabetes control.

During her interview, the observed site's nutritionist explained that one major barrier she confronted was that many patients attended nutrition appointments merely to comply with the doctor's recommendation but did not show interest or fully understood the importance of making changes. "We try to take the patient to the health educator first, 
so that he/she understands why these changes are important," she added. The health educator pointed out that patients with uncontrolled diabetes and/or hypertension received education about their conditions, behavior changes, and the importance of adherence to treatment.

\section{Organizational System and Organizational Culture}

\section{Organizational Structure}

Observations, documents, and interviews provided information about the complexity of the health center's organizational structure. While the administrative structure of the first $\mathrm{CHC}$ was highly complex due to the numerous departments across the organization, this second CHC's organizational structure seemed challenged by the large number of delivery sites. For instance, the observed site, a medium-sized organization with more than 50 employees and its own administration team, was only one of the health center's seven delivery sites. In addition, the organization, as observed during the visit, had a central administration team working at a separate location. The organizational chart indicated that the $\mathrm{CHC}$ is led by a Board of Directors, which delegated the organization's administration to the Executive Director. The health center's key management was also constituted by the Medical Director, Director of Operations, Finance Director, Associate Director, Corporate Compliance Officer, and the Human Resources Director.

As noticed in documents and confirmed with the site's director, leadership positions at service delivery sites included the site director, medical services director, a nursing supervisor, patient service supervisor, and information management supervisor. 
Site administrators and directors responded to the health center's Associate Director, while site medical directors, nursing supervisors, and allied health professionals responded to the corporation's Medical Director.

\section{Organizational, Leadership, and Learning Culture}

The data collected through observations, organizational documents, and interviews revealed multiple efforts focused on the establishment of a patient service culture. Reports indicated that the CHC developed a Customer Service Policy and trained its staff in strategies to address patient needs, including retreats and workshops for health center executives, managers, and supervisors. In addition, documents provided evidence of efforts to develop a Patient Service Department. Several interviews confirmed the central role of the Patient Service area as part of the PCMH. This patient service culture seems aligned with the establishment of a medical home coordinator network as a strategy to provide patient-centered care in Case 1.

In addition to their care coordination efforts, observed dynamics of patient service officers provided a glimpse of their interactions with patients. These staff members seemed mostly courteous and attentive to patients' needs. Staff members at the site exhibited a sense of personal responsibility for improving patient experience and outcomes. The site director, for example, was seen interacting with several patients, making herself accessible to them and requesting their feedback regarding events and services. This was also evident during interviews with the health educator, nutritionist, and nursing supervisor.

Moreover, interviews revealed changes that occurred in the health center's culture resulting from the implementation of the PCMH model. For instance, the health educator 
and PCMH leader underscored physicians' openness to working as part of a

multidisciplinary health care team. The health educator said: "I have been here rotating for a year and a half and have seen doctors more open to referring patients for health education." Both the health educator and the PCMH leader emphasized that the health center's leadership reinforced this team culture.

The CHC's leadership culture was noticeable during observations, interviews, and document review. While sites had their own management teams and were able to make decisions at the site, according to interviewees, most efforts were standardized and guided by the central administration. Nonetheless, as observed on a poster at the site and documents reviewed, the corporate administration promoted leadership development by providing training in executive and organizational coaching for its employees. One outstanding feature observed in $\mathrm{CHC} 1$ but not as noticeable in $\mathrm{CHC} 2$ was the high level of involvement of top health center executives in PCMH implementation.

Reviewed documents evidenced the health center's efforts to promote learning and performance improvement, including care coordination training for health care team members and PCMH training for staff across the organization. The PCMH leader discussed the organization's focus on improving quality of care and organizational performance. Organizational documents corroborated the use of quality improvement strategies such as the adoption and dissemination of clinical guidelines, the involvement of health care team members in performance evaluation processes, and the implementation of Plan-Do-Study-Act (PDSA) cycles, a practice confirmed by the CHC's Quality Manager. The PCMH leader also highlighted efforts to tack and monitor 
PCMH implementation through the use of the Record Review Workbook (RRWB), PCMCH self-assessment tools, and other tools developed internally.

\section{Strategic Partnerships}

According to data from organizational documents and interviews, the $\mathrm{CHC}$ had taken advantage of collaborative opportunities with partners to expand provision of services, reach out to community members, and improve performance in areas such as chronic disease management, similar to what was observed in Case 1. As read in several reports, the $\mathrm{CHC}$ sustained partnerships with other $\mathrm{CHCs}$, the Puerto Rico Department of Health, the local Medicaid program, the PR Department of Education, and the Department of the Family. The health center's O\&E Policy also stated that the organization established collaborations with hospitals, pharmacies, businesses, public agencies, nonprofit organizations, public housing projects, churches, schools, universities, and media outlets. Several interviewees confirmed the importance of these partnerships in involving the community in a variety of community events.

\section{Use of Technology to Facilitate Patient Care and Manage Population Health}

Most of the documents reviewed provided evidence of the organization's investment in health information technologies to support PCMH implementation. During the observation period, patient service staff were seen using the information systems actively to manage patient registration and appointments. According to PCMH selfassessments and sampled EHR screenshots, the CHC's electronic health record (EHR) supported care coordination through a systematic process to document patient care and track preventive procedures, lab tests, orders, referrals, and electronic prescriptions. The 
system also allowed documentation of emergency room visits and hospitalizations. Documents also highlighted the system's capacity to report quality measures, populationbased data, health risks, and information needs of patients and families.

As shared by the health educator, the EHR facilitated assessment of patients' needs. She added that the EHR provided health care team members with information that patients might not disclose during the visit. For instance, the educator highlighted:

"The patient may not want to tell me that six months ago he/she had a toe amputated and would like a shoe that is not as tight. Having access to the electronic record, I learn about this and can address the issue."

The nutritionist stressed the EHR's role in documenting recommendations, indicating that it supported tasks such as tracking compliance with appointments and patient follow-up. According to the nursing supervisor, the system allowed her to make notes about patients' issues and track gaps in preventive care and disease management processes. The EHR also seemed to have a prominent role as part of the implementation of the PCMH in the first health center studied, especially as a patient care support tool. One EHR feature not observed in this second case was the use of the system for patient engagement and communication through the patient portal.

\section{Medical Home Implementation}

\section{Approach to Patient-Centeredness}

Observations at the visited site and interviews with key informants validated the organization's focus on patient-centeredness. As observed at the site, the CHC had focused on expanding the availability of services to address the needs of its patients. The 
observed site had most of the health center's services available under one roof and encouraged patients to accommodate multiple appointments in one day to take care of their different needs. Similar to Case 1, according to the CHC2's PCMH brochure, PCMH was considered a multidisciplinary approach to provide comprehensive care and provide coordinated services.

Several interviewees expressed their views regarding the CHC's "patientcenteredness". According to the nutritionist, the organization was "focused on working for the patient's well-being." She added that the model provided an opportunity to get to know and establish a relationship with the patient and his/her family. The nursing supervisor indicated that patient-centered care involved understanding patient needs and how his/her environment affects the patient's treatment and compliance. She added: "Being 'patient-centered' means being focused on the fact that the patient must have all the necessary elements to maintain his/her health."

\section{Approach to PCMH Implementation}

As explained by the health center's PCMH leader and observed in several organizational reports, the $\mathrm{CHC}$ initially pursued an individual-site $\mathrm{PCMH}$ implementation, rather than a multi-site or organizational approach like the one implemented at the first health center. According to reports and the input of the PCMH leader, the first site was recognized as a level 2 PCMH in 2014. The second site, the one observed, received recognition in 2015. The PCMH leader clarified that this second site was re-accredited in 2017, whereas the first site was not prepared to re-certify when its recognition expired in 2016. However, according to the PCMH leader and the health educator, the site continued implementing the standards of the model. 
The PCMH leader indicated that the PCMH implementation process required the development of multiple new norms and many decision-making processes. Among the first was the designation of a leader and implementation teams. This staff member indicated that the central committee included a Board member to ensure staff buy-in, the executive director, and the medical director, the main support at the central level. The organization also established a sub-committee at the site level constituted by site supervisors, managers, and directors. She heightened the leading role of the observed site's nursing supervisor in the implementation. Reports also showed that the organization prepared a group of "super users" and champions to support implementation of the EHR across the organization.

The PCMH leader clarified that, in order to maintain a similar model throughout the $\mathrm{CHC}$, even though only two sites were officially seeking PCMH recognition, all sites were guided towards the implementation of the minimum required standards. Even when the PCMH structure was not implemented in every site and department throughout the organization, such as in Case 1, along the way, staff across sites developed a culture aligned with the PCMH model. She explained that commitment to patient care and patient service improved. She said the staff was trained on how to educate patients about the importance of complying with appointments and adhering to treatment. The PCMH leader added that nurses played a fundamental role.

Documents reporting the PCMH implementation process and other organizational reports revealed efforts by the CHC to maximize staff availability to support PCMH implementation by, for instance, revising basic salaries and benefits to improve retention and recruitment and maximize opportunities to have full functioning health care teams. 
The health educator explained that the organization also implemented physical infrastructure and patient flow projects aligned with the model and continued to recruit health professionals to complete the teams.

During her interview, the PCMH leader indicated that the CHC's leadership had recently decided not to implement standards established by the accrediting agency they originally selected, since they were interested in pursuing a PCMH recognition at the organizational level. She explained that monitoring of PCMH standards under the agency's model had been discontinued, but sites would still implement the basic principles, which were aligned with the requirements of HRSA's Health Center Program.

\section{Challenges and Barriers to Implementation}

Site observations, interviews, and document reviews identified a series of challenges and barriers to PCMH implementation at this $\mathrm{CHC}$. According to information obtained from all sources, staffing seemed to be the greatest challenge for this organization. As reported in several documents and confirmed by interviewees, the sites needed to recruit more staff and reduce staff turnover in order to have complete health care teams, balance the workload, and enhance patients' access. According to the health educator, the lack of full-time health education staff was a major barrier. The health educator expressed that, given all her responsibilities, she was only able to see 10 patients on average during her three days at the observed site. Other interviewees agreed and indicated similar situations whereby staff was present only once weekly. As in Case 1, in addressing many of these challenges, $\mathrm{CHC} 1$ opted for establishing a network of medical home coordinators with nursing background available to all providers. 
Another challenge documented in a report on the status of the implementation was lack of consistency among patient service staff in complying with appointment policies. The report also highlighted the large number of patients scheduled per day per provider, which limited providers' capacity and time to comply with PCMH requirements. Additionally, the report stressed patients' lack of compliance with appointments, nonadherence to treatment and recommendations, and social barriers as major challenges to achieving the successful implementation of the PCMH.

According to interviewees, frequent changes at the site's administration level was a fundamental problem. The health educator stated that administrator changes affected progress in the implementation of the model, especially if the leader came from a site not recognized as PCMH. This staff member also brought up challenges arising from having to comply with different health insurance companies administrating the local Medicaid program. In addition, since not all sites were implementing the PCMH model, staff had to employ different strategies.

The nursing supervisor noted issues with the EHR system's capacity to automatically integrate different processes. If the organization lacked the necessary interfaces, those implementing the model could encounter multiple obstacles, she noted. Patient communication with the site represented another challenge, according to the nursing supervisor. There had been many complaints from patients indicating they were not able to make appointments. This affected the process because patients ended up getting to the site as walk-in/same-day.

The PCMH leader discussed multiple challenges to PCMH implementation, based on her experience with the process. One key challenge was the lack of commitment of 
PCMH champions at the site to follow-up with team members on the implementation of the model. Another challenge was non-compliance with structured daily "team huddles". She explained that some staff did not want to meet regularly and document the meetings. Additionally, the fact that some sites were not recognized as PCMH and others were generated concerns among those working at PCMH recognized sites. Furthermore, sites not recognized as PCMH were not as committed to the model or following the guidance provided by the PCMH leader.

\section{PCMH Implementation Facilitators}

Several key informants stressed the importance of interprofessional communication as a fundamental factor to the success of PCMH implementation. Communication between professionals, as expressed by the health educator, was key to achieving improvements in the health condition and patient quality of life. Furthermore, the health educator emphasized the importance of physicians acknowledging the role of a multidisciplinary team and the involvement of other health professionals in the care process. The nursing supervisor highlighted the advantage of having young nurses and staff members well-trained in computer systems and the operation of a health care system with multiple health services on site.

Every single interviewee pointed to the essential role of the EHR in the implementation of the PCMH model, an observation also made by key informants at the first health center. The PCMH leader mentioned the system's ability to collect and organize massive amounts of data for numerous performance measures. The nutritionist stressed the role of the EHR in facilitating patient follow-up. According to the health educator, the EHR allowed health care team members to monitor patient flow and patient 
progress. The nursing supervisor highlighted the opportunity to identify and document gaps in preventive care and health maintenance procedures.

The PCMH leader mentioned that many PCMH standards were tied to HRSA requirements, which facilitated implementation and compliance. She explained that patient-centered care is the essence of the CHCs and what the health center did was refine what the organization already had, developing additional standards and policies as needed. Additionally, she explained that most of the preventive and chronic disease measures required by the PCMH were already part of what CHCs report as part of HRSA’s Uniform Data System.

Finally, the PCMH leader stressed the role of nurses in the implementation of the model, as they ensured that patients had all the health care plan requirements and PCMH documentation was complete. This is highly comparable to the facilitating role of medical home coordinators in Case 1, mentioned frequently by study informants at this organization. Moreover, according to CHC2's PCMH leader, every nursing supervisor at this health center had the authority to make decisions at his/her site, which supported behavior modification strategies among staff members. The PCMH leader affirmed that nursing supervisors were highly committed to ensuring their staff collaborated with physicians in complying with PCMH standards.

\section{Summary of Key Findings and Organizational Factors Identified}

This section summarizes the salient features of the implementation of the PCMH model observed in Case 2. It also presents the major challenges and facilitators to the implementation of the PCMH model at this health organization, as revealed by study findings. 


\section{Salient Features of CHC2's PCMH Model}

- Service delivery model. The CHC provided multiple health and patient support services under one structure, improving access to care opportunities. While no specialists were available on site, several specialty care services were available within the CHC network of delivery sites.

- Patient-centered culture. A key feature of this CHC's PCMH model was the establishment of a Patient Service department constituted by a group of patient service officers central to the PCMH strategy. The CHC placed emphasis on patient-centered care, patient engagement, and the establishment of a patient service culture. Leaders and staff shared a clear vision of patient-centered care.

- Multidisciplinary approach. Whereas the physician-nurse partnership was dominant in this organization, the $\mathrm{CHC}$ 's leadership invested in the recruitment of health educators, nutritionists, social workers, and patient service officers to address patient care needs through a multidisciplinary approach.

- Key leadership and coordination role of nurses. Nurses at this CHC had a prominent role in the patient care process and provided fundamental guidance to other members of the health care team. Furthermore, nursing supervisors had authority to make decisions at the site level and conducted efforts to ensure staff commitment to PCMH implementation.

- Access to care. Access to care was a major priority for this CHC. The health center established an Outpatient Department (OPD) exclusively to attend walk-in and same-day patients, which helped balance patient load in the regular clinics. 
The organization also made improvements to patient flow and its facilities to further address patient needs enhance access to care.

- Learning culture and performance improvement. This $\mathrm{CHC}$ had a structured performance improvement program with emphasis on quality of care. During PCMH implementation, the organization focused on staff training across the organization and the establishment of guidelines and processes to assess and improve PCMH implementation.

- Strategic partnerships. The CHC took advantage of collaborative opportunities with partners to expand provision of services, reach out to community members, and improve performance in areas such as chronic disease management.

- Central role of the EHR. The incorporation of the electronic health record (EHR) was fundamental to patient care, care coordination, patient follow-up, identification of health needs, and compliance with PCMH requirements.

- Incorporation of chronic disease management to the PCMH strategy. Members of the health care team, particularly nurses and other allied health professionals, monitored patients with chronic diseases, identified education needs, and provided self-care support on site and at home.

- Emphasis on patient education and communication. The CHC incorporated multiple opportunities for individual, group, and community education, placing emphasis on major chronic diseases affecting the patient population. The health center also maintained ongoing communication with patients and community members through different organizational and health communications and health center and community events. 


\section{Summary of Challenges and Facilitators to PCMH Implementation}

Table 11 summarizes the major challenges and facilitators to PCMH implementation identified during the research process at $\mathrm{CHC} 2$.

\section{Table 11.}

Summary of Challenges and Facilitators to PCMH Implementation at CHC2

\begin{tabular}{|c|c|}
\hline Challenges & $\begin{array}{l}\text { a. Large number of delivery sites, each with its own management and } \\
\text { staff structure, challenged PCMH implementation at multiple sites. } \\
\text { b. The selection of an individual site PCMH approach restricted } \\
\text { opportunities to spread the initiative to other sites and generated } \\
\text { concerns among those working at PCMH recognized sites. } \\
\text { c. Shortage of health education staff limited the CHC's capacity to } \\
\text { provide individualized education to its large number of patients with } \\
\text { chronic diseases. } \\
\text { d. Shortage of patient service staff affected strategies to enhance } \\
\text { access to care and care coordination. } \\
\text { e. Shortage issues prompted staff sharing among sites to complete the } \\
\text { multidisciplinary teams needed to implement the model. } \\
\text { f. Lack of a firm policy establishing the health care team structure. } \\
\text { g. The CHC did not enforce a specific policy or structure for "team } \\
\text { huddles" or other type of health care team meetings. } \\
\text { h. Many care coordination responsibilities under the PCMH model } \\
\text { were assigned to nurses, in addition to the many roles they had. } \\
\text { i. Patients' lack of compliance with appointments, lack of interest in } \\
\text { making changes, and non-adherence to treatment affected chronic } \\
\text { disease management efforts. } \\
\text { j. PCMH implementation efforts at the site level diluted when } \\
\text { ongoing monitoring and follow up from the PCMH leader stopped. } \\
\text { k. Implementation progress was affected by the lack of commitment of } \\
\text { the sites' PCMH champions to follow-up with team members. } \\
\text { 1. Inconsistency among patient service staff in complying with } \\
\text { appointment policies caused large number of appointments per day, } \\
\text { limiting providers' capacity to comply with PCMH requirements. } \\
\text { m. Frequent changes at the site's administration level affected progress } \\
\text { of PCMH implementation, especially if the leader came from a site } \\
\text { not recognized as PCMH. }\end{array}$ \\
\hline
\end{tabular}




\begin{tabular}{|l|l|}
\hline n. & The EHR's capacity to automatically integrate different processes \\
& was limited by the lack of necessary interfaces in place. \\
o. & Issues with communication systems affected phone access to the \\
& CHC and resulted in an increase in walk-in/same-day patients.
\end{tabular}




\section{CHAPTER V DISCUSSION AND CONCLUSION}

The Health Center Program of the Health Resources and Services Administration (HRSA) was established as a response from the federal government to provide access to health care to uninsured and underserved populations. Starting with only two health centers in 1965 , today the nearly 1,400 HRSA-funded community health centers (CHCs) provide care and support to over 27 million people in the US and its territories (HRSA, 2018a). CHCs serve 1 in every 3 low-income uninsured individuals in the US and 1 in every 6 Medicaid beneficiaries (NACHC, 2017). Most of these individuals are not only largely affected by poverty and other social ills, but many of them live with multiple chronic diseases.

CHCs are ideal settings for public health practitioners to develop and implement targeted behavioral health interventions. These interventions have the potential of improving the quality of lives of millions of people across the nation with chronic diseases. Establishing partnerships to support chronic disease prevention and management efforts at $\mathrm{CHCs}$ could help prevent multiple complications. Conditions such as diabetes and hypertension are important contributors to premature mortality, loss of quality of life, and loss of productive years.

CHCs are an essential component of the US health system and play a key role in helping accomplish the nation's health goals. Given their role in the fight against chronic diseases, it is necessary to pay attention to how well these organizations are moving toward the accomplishment of their mission and examine factors that can improve their performance in this endeavor. Addressing the needs of underserved populations affected 
by chronic conditions is essential to Public Health, a field that in the last decades has become increasingly aware of the importance of monitoring these diseases to improve the health of the population.

\section{Summary of the Research}

While research about the PCMH model and the implementation of its standards and principles of this model across different health systems has been extensive, there was a need to understand why anticipated PCMH outcomes with regards to chronic disease management have not been equally achieved among CHCs, why PCMH has only seemed to work effectively at certain CHCs, and what characterizes successful PCMH models at these safety net organizations. As discussed thoroughly in Chapter 1, several researchers proposed that variations in CHC performance and PCMH implementation could be the result of the influence of factors beyond PCMH principles and HRSA support, including organizational context.

The purpose of this study was to explore and describe the organizational factors that contribute to the successful implementation of the PCMH model and improvement of chronic disease management outcomes among HRSA-funded CHCs with PCMH recognition. This research explored and characterized organizational factors that distinguish or affect PCMH implementation at two CHCs with PCMH status recognized by HRSA as high-quality leaders with variations in organizational context with three objectives in mind:

1) to compare and contrast organizational factors identified in the two participating CHCs; 
2) to describe how these $\mathrm{CHCs}$ have implemented PCMH elements related to chronic disease management, mainly diabetes and hypertension control; and 3) to propose an organizational model to support chronic disease management and PMCH implementation among HRSA-funded CHCs using theoretically-framed interpretations.

The study focused mainly on "inner setting" organizational factors involved in PCMH implementation and the "process of implementation, two domains of the Consolidated Framework for Implementation Research (CFIR), discussed earlier in the Introduction. A qualitative collective case study was designed and implemented to support the accomplishment of the study's aim and objectives. Two CHCs with PCMH status, both recognized by HRSA as top-quality performers, but with marked differences in organizational contexts, were included as part of this study. One of these health centers was located in South Florida and one in Puerto Rico. As presented in chapters 3 and 4 and further presented in the forthcoming discussion, observed differences in patient and staff mix, organizational cultures, patient-provider/staff interactions, and practices, among others, provided an instrumental opportunity to explore the organizational adaptation of the PCMH implementation.

In addition to having PCMH recognition, both organizations represented a desired level of performance and quality of care. At the time of the study, these CHCs were designated "Health Center Quality Leaders" for achieving the best overall performance among health centers (HRSA, 2018b). The two CHCs had also been recognized as "National Quality Leaders" for exceeding national benchmarks for chronic disease management, preventive care, or perinatal/prenatal care, including Healthy People 2020 
goals (HRSA, 2018b). This was fundamental to expand the potential of finding and exploring successful models of PCMH implementation at CHCs.

The case study employed three data collection methods: field observations, interviews, and documentation review/analysis. This process involved 70 hours of field observation, 35 at each site, and close to 14 hours of interviews with a total of 13 health center staff members. Data collected and themes emerging from the data analysis allowed the development of two individual cases (chapters 3 and 4) that characterize both participating organizations, their organizational cultures and dynamics, key actors, PCMH implementation practices, and chronic disease management strategies.

\section{Discussion}

This section integrates and compares key findings from both cases to address the main inquiries leading to this study. The focal point of this discussion is the presentation of organizational level aspects, characteristics, and dynamics that contribute to or hinder the capacity of the organizations studied to implement the PCMH model and bring about desired chronic disease outcomes among the populations served. The discussion also reviews key findings in light of the existing $\mathrm{PCMH}$ implementation literature and two fundamental theoretical frameworks: The Expanded Chronic Care Model (ECCM) and the Consolidated Framework for Implementation Research (CFIR). The ECCM supports the analysis of findings regarding PCMH implementation as they relate to CHCs' efforts to improve chronic disease outcomes. The CFIR provides a basis for the discussion of the "inner setting" characteristics and "implementation processes" that facilitate or challenge the implementation (Damschroder et al., 2013). 
As documented earlier, the two cases included in this study were two high-quality CHCs implementing the PCMH model, one located in a densely populated, metropolitan community in South Florida (Case 1) and the second one in a rural, mountainous town in Puerto Rico (Case 2). Case 1 provides services across three main delivery sites within a 16-mile distance, while Case 2 delivers services at seven sites located in six different municipalities in Puerto Rico. Both organizations were founded over 40 years ago and serve a similar number of patients.

One key characteristic of CHCs operating in Puerto Rico is the homogeneity of its population. Close to $99 \%$ of patients served by $\mathrm{CHC} 2$ are Hispanic/Latino (HRSA, 2018c). The majority are Puerto Rican. This level of homogeneity and cultural uniqueness is highly unlikely at other CHCs across the continental states. This could be an advantage when planning and designing strategies for this population, especially educational programs. It was interesting to observe patients at the Puerto Rico site holding lively exchanges as friends, whereas patients at the South Florida site were unlikely to engage in conversations. Given the cultural homogeneity in Case 2, found in both patients and staff, staff members were able to use a familiar and colloquial communication style with patients. This may be more difficult to achieve at a highly diverse organization such as Case 1, where observed patients and staff were culturally diverse.

The ethnic differences observed above, however, did not seem to influence the implementation of the PCMH model, delivery of services, or patient care processes. For example, both of the organizations studied demonstrated optimal performance, as evidenced by recognitions awarded by HRSA. In addition, PCMH recognition had also 
been achieved by both organizations. The implementation of multiple PCMH standards and principles was clearly observed and documented throughout the study.

This research provided a comprehensive view of CHCs' efforts in multiple areas aligned with PCMH standards and principles established by different accrediting agencies, many of which emerged as themes in this study. As discussed in chapters 3 and 4, both organizations provided comprehensive services, offered patient support and enabling services, implemented efforts to enhance access to care, placed emphasis on care coordination, team-based care, planned care, and used technology information systems to support these interventions. These CHCs have also incorporated multiple elements of the Chronic Care Model and the Expanded Chronic Care Model, including self-management support, community partnerships, clinical decision support systems, and proactive teams (Barr et al., 2003). Yet, whereas there were similarities identified in the implementation of these standards among participating CHCs, there were important differences noted in the implementation of the model, a variability documented previously by PCMH researchers. In fact, as discussed in Chapter 1, researchers have noted that the high level of variability in the implementation of the model obstructed exploring the impact of the PCMH on health care practices and health outcomes (Jackson et al., 2013; Morgan et al., 2014; Davy et al., 2015; Shippee et al. 2017; Quinn et al., 2013; and Hoff, 2010).

Both participating CHCs provided a vast array of health services to address the needs of the communities served, including enabling services to minimize barriers to care. As HRSA-funded CHCs, both offered services to all patients regardless of their ability to pay. Another key finding among the two CHCs was the use of strategies to 
enhance access to care through a variety of approaches, including walk-in/same-day appointments. Case 2, for instance, implemented a formal approach to serve walk-in or same day patients through an Outpatient Department (OPD) exclusively for these patients, which helped balance patient load in the regularly scheduled clinics. This is not only a way of addressing the needs and preferences of the population served, as established by the PCMH standard of "Access to Care," but is also crucial in ensuring enough time for health maintenance and chronic disease patients, who require ongoing monitoring, self-care support, and education.

As observed during the field research process and discussed with key informants at this health center, to facilitate the care coordination practices required to provide integrated care, $\mathrm{CHC} 1$ invested in the establishment of a network of medical home coordinators (MHCs) and referral coordinators (RCs). CHC2 relied primarily on nurses to coordinate and track all patient care processes and a patient service area to handle scheduling, registration, and authorizations. In both $\mathrm{CHCs}$, nurses played a leading role in managing the entire care coordination process. Yet, contrary to what was observed in Case 1, CHC2 patients were responsible for coordinating appointments with external providers themselves. This could be a barrier to the completion of the patient care cycle as patients could find it difficult to manage the complexities of the health care system. The health care system in Puerto Rico is highly complex and fragmented. It requires patients to move from provider to provider and in between administrative offices to obtain the referrals and paperwork needed to access a health service. As shared by several health center key informants, this was the reality for many beneficiaries of the local 
government health insurance plan, who constituted the majority of patients seen by CHCs in this US Territory.

Findings regarding the composition and dynamics of health care teams are also worth discussing, especially considering that team-based care is not only a major PCMH element, but also a key ingredient to achieve coordinated care. Furthermore, according to the CCM, improved chronic disease outcomes are the result of interactions between activated patients and proactive teams of health care providers and other health professionals (Barr et al., 2003). Case 1 placed emphasis on the consistent availability of health care teams across the organization, based on a fixed team structure. Case 2 seemed to prioritize the physician-nurse partnership, aided by a team of allied health professionals, including a health educator and nutritionist.

Whether organized as part of a structured health care team or as integrated services within the system, the availability of multidisciplinary health professionals seemed fundamental in the implementation of chronic disease management at both organizations. For instance, at $\mathrm{CHC} 1$, the work of medical home coordinators (MHCs), the daily interactions between health care team members, and interactions during the observed "team huddle" supported the ongoing focus of the diabetes management area on maintaining control of this condition and supporting the patient in this process. The members of the health care team monitored, tracked, and followed up with patients on a continuing basis. The different members of the team, MHCs, the leading physician, the nurse, and the dietitian, provided both individual and group education regularly to these patients. At the Puerto Rico site, the health educator, nutritionist, and social worker collaborated with the primary care provider and the nurse to follow-up with patients 
affected by chronic diseases, frequently working jointly to discuss alternatives to address patients' circumstances.

The CCM and ECCM place special emphasis on activating the role of patients in managing their own care through self-management support (Barr et al., 2003). This element of the CCM and ECCM underscores the importance of developing skills for health and wellness among patients with chronic disease and, in the case of the ECCM,

the community. This educational approach to self-management support was evident in the two participating CHCs. Both offered individual and group education to patients with chronic disease, particularly those with uncontrolled glucose and blood pressure levels, as discussed earlier. In Case 1, diabetes self-management support occurred within the structure of an area specialized in diabetes management that incorporated a network of onsite specialists necessary to maintain diabetes control. At $\mathrm{CHC} 2$, while diabetes management was generally guided by the provider-nurse team, the health educator, nutritionist, and social worker were central figures.

\section{Organizational Factors}

According to the CFIR, crucial "inner setting" factors play vital roles in the implementation of a project or intervention (Damschroder et al., 2013). These factors include the organization's culture, networks among organizational agents, staff attitudes and behaviors, the level of commitment to changes, and the availability of resources. As observed throughout this study, the implementation of the PCMH model at participating CHCs seemed influenced by a series of structural factors. Contrary to what is generally expected, culture did not appear to have an impact on the way the model is implemented or its performance. 
After exploring multiple organizational dynamics and processes, organizational structure emerged as an important factor involved in PCMH implementation. Both of these organizations had highly complex organizational structures, which could increase the difficulty of the implementation of organizational transformation processes such as the PCMH. Case 2's organizational structure seemed further challenged by the large number of delivery sites, each with its own managerial structure in addition to the corporate central administration. This organizational structure, combined with the choice of an individual site implementation, as documented in Case 2, seems to have affected this organization's capacity to implement the PCMH model across the organization.

It seems important to highlight the PCMH structure established at $\mathrm{CHC} 1$. PCMH at this health center was managed as a program with its own policies and structure, supported by health care teams from every department, including a network of medical home coordinators and referral coordinators. CHC2, on the other hand, managed PCMH as an independent quality project guided by a central level committee and site level subcommittees, but not as an organizational intervention. PCMH implementation seemed isolated from other health center initiatives. During interviews, this health center's PCMH leader discussed the planning process involved and her role in what she frequently referred to as "the project." Furthermore, when asked about the participation of the "Quality Department" in the implementation of the PCMH, she explained that quality improvement initiatives conducted as part of the $\mathrm{PCMH}$ were taking place independently.

Undoubtedly, these two CHCs manifested a patient-centered culture, making "patient-centeredness" part of their organizational cultures. These organizations placed 
emphasis on providing services to address patient and community needs identified, as required by the Health Center Program (HRSA, 2018b). In addition, both cases exhibited patient-centered service delivery models and interactions. Moreover, Case 1 made the PCMH part of its organizational operation through the establishment of a PCMH program implemented across the organization. Case 2 focused on the establishment of what they called a "patient service culture," which included the establishment of a patient service department and a patient service policy for all health center sites. Within a PCMH, these practices are expected to improve the patient's experience and engagement in care (Luxford, Safran \& Delbanco, 2011; Beacham et al., 2012; Platonova et al., 2016). Other inner setting characteristics observed included leadership and learning cultures. Even when the two CHCs exhibited flexibility in regard to decision-making processes at the patient care level, both had clear lines of authority and well-established leadership structures. One particularity observed in Case 1 was the high level of involvement of top-level executives in the implementation of the PCMH model, from adoption to daily monitoring. For example, as discussed in Chapter 3, the CEO and the $\mathrm{COO}$ made rounds every morning, looking at everything, from lighting to how patient care was delivered. According to this health center's CEO, this made a difference because "the staff knows that there is someone watching and making sure that they are going the extra mile and gives them credit for going that extra mile."

The above type of oversight contrasts with $\mathrm{CHC}$, where the PCMH leader's monitoring stopped after the central administration decided to follow a new accreditation process. As shared by the PCMH leader, once the organization decided to pursue PCMH recognition through a different accrediting organization, with different PCMH guidelines, 
she was asked not to continue monitoring the site's compliance with PCMH interventions until a new agency was selected. The autonomy to select from various accrediting organizations, while providing flexibility and self-determination to the PCMH, also contributes to instability in the implementation and monitoring of programs. Even when programs are working well, a transfer to another accrediting organization with its own set of goals and procedures leads to the fragmentation of what otherwise were stable practices and programs at the PCMH. This becomes especially disrupting to established practices and programs related to the control and management of chronic care that require continuity of implementation and evaluation. The openness to withdraw from one accrediting agency to another is structural and external to the PCMH culture and its own organization; however, one that contributes to fragmentation of programs and monitoring at the PCMH.

Despite differences noted with respect to leadership culture, both CHCs shared similarities in their learning cultures. These centers placed major emphasis on performance and quality improvement and had established structured quality improvement programs, possibly due to the fact that this is a key priority for HRSA. According to the Health Center Program requirements, CHCs must have an ongoing quality improvement/assurance (QI/QA) system and establish the necessary organizational processes to support the quality assurance program and provision of highquality patient care (HRSA, 2018d). In addition, CHCs must demonstrate improvement in key preventive care and chronic disease performance measures, among others. 


\section{Summary of Organizational Barriers and Facilitators}

In addition to comparing and contrasting organizational factors in the two participating CHCs, this study helped identify a series of organizational barriers and facilitators involved in the implementation of the PCMH model. The identification of barriers and facilitators can provide guidance on strategies to adapt the PCMH to different CHC settings, a major driver of this study and a concern shared by other researchers (Hoff et al., 2012).

\section{Challenges and Barriers to Implementation}

Processes and resources required to implement the model, including financial, human, and time resources could pose significant challenges for CHCs. Both cases studied made staff investments to ensure they had complete health care teams. They also invested in health information technology infrastructure and systems and, in the case of Case 1, an entire communication system due to issues affecting patient communication with the CHC. While the investment in the Electronic Health Record (EHR) seemed to be a major facilitator, according to Case 2, it can also pose challenges to care coordination practices if the system does not have all necessary capabilities.

Staff shortage and challenges to fill certain necessary positions were also stressed by the two CHCs. This is especially important to the management of chronic conditions, including diabetes and hypertension, which require ongoing monitoring and multiple care coordination interventions. Both CHCs pointed out the impact of staff shortage on care coordination, self-management support, providers' workload, and access to care. For example, key informants at these CHCs expressed concerns over care coordination challenges resulting from the medical home coordinators' and nurses' heavy workload, 
especially when they managed and monitored every event in the patient care cycle.

MHCs interviewed at $\mathrm{CHC1}$, who were in charge of coordinating the entire patient care process, follow-up with patients, and provide health education, explained that they each supported four primary care providers and frequently had to back other MHCs. CHC2's nursing supervisor stressed that care coordination responsibilities were assigned to nurses, "on top of the many roles they already have." In addition, the health educator at this site highlighted that she had to provide services at two different sites and that her limited time only allowed her to see 10 patients weekly at the observed site.

Finally, study findings revealed a second important structural barrier resulting from the flexibility to select one or another of the PCMH implementation approaches. For instance, pursuing an individual-site instead of a multiple-site or organizational approach can lead to lack of commitment among sites not recognized. Additionally, site observations and interviews at $\mathrm{CHC} 2$ informed on perceptions among health professionals in the PCMH-site regarding their heavy workloads, which they thought to be highly demanding when compared to expectations at non-accredited sites. Key informants in Case 2 also brought up the consequences of frequent changes in the site's administration on the continuity and progress of the implementation of the model, as pointed out above, alongside the lack of commitment of PCMH champions at the site level to follow-up with team members. Lastly and important, as discussed in Case 2, a multiple-site implementation can be highly difficult and challenging; however, this challenge, as well as fragmentation in some programs resulting from transitions in accrediting agencies, are structural and beyond the local PCMH site. 


\section{PCMH Implementation Facilitators}

Based on the experience of Case 1, compared to Case 2, establishing the model at an organizational level, rather than an individual site implementation, seems to be a more effective approach to $\mathrm{PCMH}$ implementation. $\mathrm{CHC1}$ 's system-wide model helped facilitate dissemination of the patient-centered culture and standardization of the implementation of PCMH standards across health center departments. As observed during the site visit and discussed with health center key informants, every department worked as a PCMH itself. Moreover, $\mathrm{CHC1}$ seemed to have found a highly efficient strategy by incorporating a network of MHCs, all licensed practical nurses, and referral coordinators. This not only gave continuity to the implementation of the model, but also provided a structure for care coordination practices in every department, which was ultimately favorable to chronic disease management efforts. The experience of both CHCs underlined the role of MHCs, nurses, nursing supervisors, and health educators, which emerged as fundamental to the implementation of patient-centered care. This is critical to the focus of this study since, as revealed by previous PCMH researchers, the PCMH model was developed as a comprehensive approach to improve chronic disease management, a process that benefits from the involvement of the nursing staff and health educators (Setodji et al., 2017, Lieberthal et al., 2017; Rivo et al., 2016; Milani \& Lavie, 2015; Holtrop, 2010; Forbes \& While, 2009; Wagner, 2000).

Leadership engagement was also identified as a facilitator, especially the high level of involvement during the PCMH recognition process and in the daily monitoring of compliance with PCMH standards, as identified in Case 1. Furthermore, based on the experience of Case 1, the availability of complete health care teams working jointly to 
make decisions about the care process, along with daily structured "team huddles", brief health care team meetings recommended as part of the PCMH approach, seemed to facilitate patient care and coordination. These practices were also facilitated by the incorporation of the EHR at the two participating CHCs. The system supports the entire patient care process and is a key tool for the ongoing monitoring and follow-up required by chronic care. Moreover, it is fundamental in managing care for patient populations.

\section{Table 12.}

Summary of organizational barriers and facilitators to $\mathrm{PCMH}$ implementation at $\mathrm{CHCs}$

\section{Organizational factors identified as barriers for PCMH implementation at CHCs}

1. Implementing the model at multiple sites individually

2. Lack of a firm policy establishing the constitution of the health care team

3. Lack of a specific policy to enforce "team huddles" or other types of multidisciplinary health care team meetings

4. Lack of ongoing monitoring and follow up

5. Lack of staff resources to implement complex processes

6. Heavy workloads on nurses and medical home coordinators

7. Lack of commitment of providers, staff, and PCMH champions

8. Making significant investments while maintaining affordable care

9. Difficulties encountered in recruiting positions needed for PCMH implementation

10. Staff shortage in positions required to implement care coordination processes

11. Impact of communications on patients' access to the health center

12. Patients' low compliance with appointments, treatment, and recommendations.

13. Lack of stability at the administration level

14. Limited Electronic Health Record capabilities

\section{Facilitators for PCMH implementation at CHCs}

1. System-wide implementation

2. Patient-centered vision and culture

3. Learning and performance/quality improvement

4. Organizational resources and supporting structures:

a. Financial resources

b. Staff resources

c. Fully implemented Health Information Systems (EHR)

d. Communication systems

e. Staff buy-in and commitment to the patient-centered culture

f. Committed champions and leaders 
g. Leadership and staff stability

h. Leadership engagement in PCMH implementation

5. Fully-functioning health care teams and structured, systematic team meetings, and frequent interprofessional communication

6. Care coordination support networks

7. Ongoing, interactive patient communication

\section{Proposed Organizational Framework for PCMH Implementation at CHCs}

In alignment with the CFIR, this study showed how multiple organizational factors interact with operational and strategic dynamics involved in the implementation of the standards and principles of the PCMH model. Following one of the study's objectives, this research proposes the consideration of a conceptual organizational framework for the implementation of the PCMH model at CHCs, based on the organizational factors identified in this analysis. Figure 3 visually organizes these elements in a proposed organizational framework.

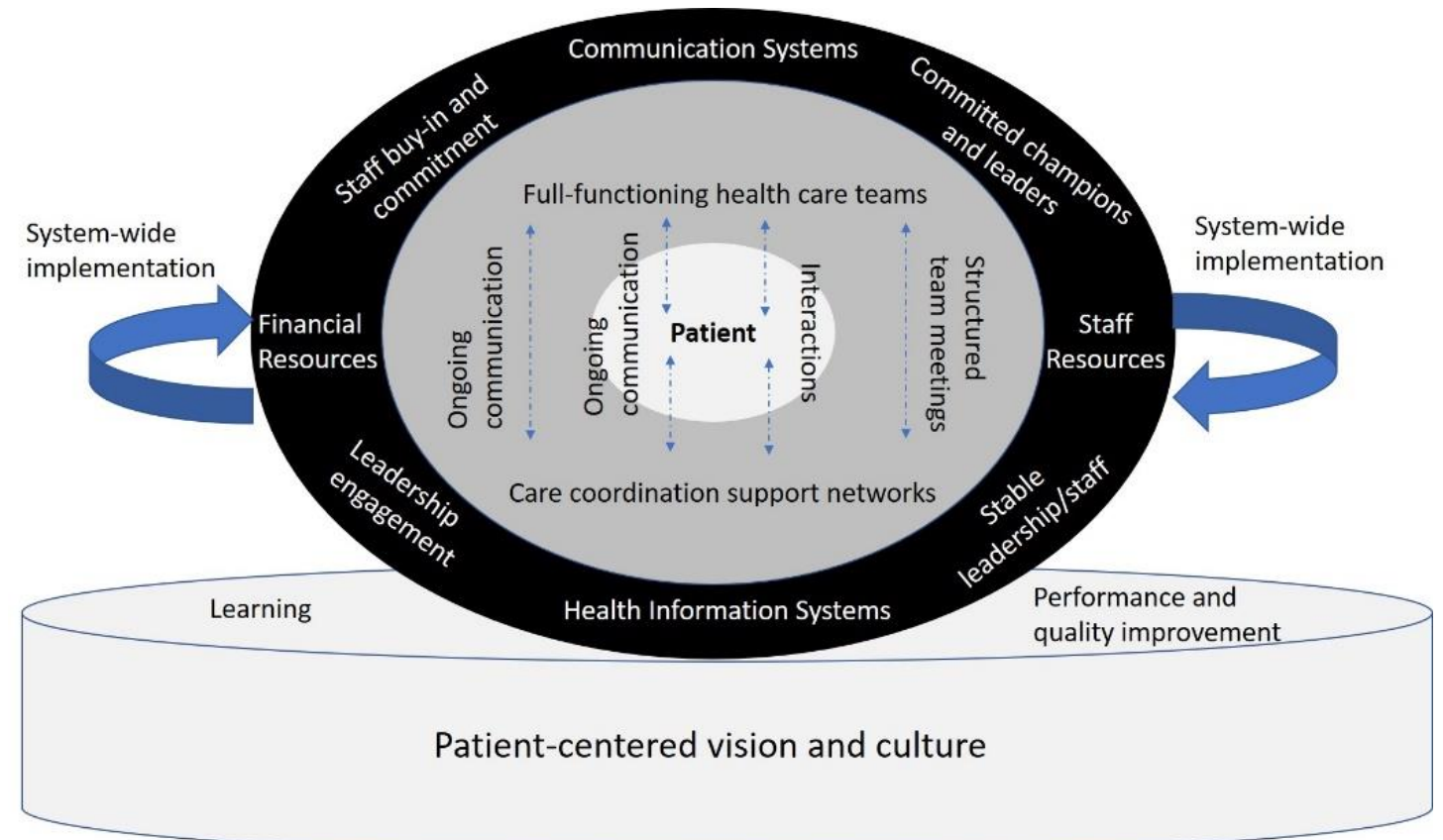

Figure 3. Proposed organizational framework for $\mathrm{PCMH}$ implementation at $\mathrm{CHCs}$ 


\section{Conclusions and Lessons Learned}

HRSA-funded CHCs across the nation seem to experience multiple challenges and barriers in the implementation of the PCMH model. For instance, CHCs confront numerous hurdles, including financial constraints, difficulties in recruiting necessary staff, high staff turnover, and high workloads (Shin et al., 2009; Rosenbaum et al., 2010; Quinn et al., 2013; Perez et al., 2013). Given their fundamental role in the nation's health care system, these organization must have the necessary internal and external supports needed to accomplish their mission of improving the lives of disadvantaged communities.

This research identified a series of organizational factors involved in the implementation of the PCMH model at HRSA-funded CHCs. These factors could be considered at the time of implementing this complex model, regardless of the particularities of the organization. Unquestionably, further research is required to determine whether or which organizational factors are associated with successful implementation of the PCMH and concomitant improvements in chronic disease outcomes. Future studies should continue looking into the effect of the implementation of the PCMH model on health outcomes, compared to the impact of the HRSA Health Center Program model. As revealed by this study, many of the accomplishments achieved by these organizations could be a result of the implementation of the Health Center Program model and the motivation to obtain HRSA recognition for the reported performance measures, rather than the actual implementation of PCMH standards.

The design of this study had several strengths and limitations. The research relied on multiple data collection methods and two cases to illustrate the phenomenon of interest, which strengthens the validity and reliability of the study. Moreover, in addition 
to ensuring that cases selected complied with specific criteria that would provide access to the phenomenon of interest, the study used the same procedures at both sites. To foster fidelity in the replication of the field work, study protocols and data collection guidelines were used and both cases were individually developed following a standardized withincase analysis protocol. It is also worth noting that, after data analysis, findings were shared separately with health center liaisons and other health center leaders as a validation strategy. This provided participating $\mathrm{CHCs}$ with the opportunity to examine findings and validate whether they accurately represented PCMH practices, barriers confronted, and facilitators identified at their health center.

It is worth considering that there may by multiple other organizational factors involved in the implementation of the PCMH model at HRSA-funded CHCs, in addition to those revealed by this study. Several limitations could have affected the opportunities to observe or note other aspects. For instance, this study may be restricted by factors such as days and times of observation, influence of the presence of the researcher, and key informants' understanding of interview questions. Furthermore, the quality and accuracy of findings may have been affected by differences in the information and levels of access provided by participating CHCs.

\section{Lessons Learned}

This study highlights the role of organizational performance in the implementation of chronic disease management and quality improvement initiatives at CHCs. Several researchers have observed that some health organizations struggle to put some of these standards or elements into practice once they receive PCMH recognition (Ottmar et al., 2015; Dobbins et al., 2018; Timbie et al., 2017). Hoff et al. (2012) stressed 
the importance of identifying the strategies or interventions necessary to achieve successful PCMH implementation and the establishment of a blueprint for the implementation of the model. While this could be extremely useful to many CHCs, these health organizations must first establish solid organizational foundations for the implementation of the model.

One key finding from this research is that the PCMH model cannot be effectively implemented without the organizational structure and management necessary to support implementation. The PCMH is an organizational model, not just a program or intervention to improve quality of care or chronic disease outcomes. These are results expected from the implementation of the model, but the focus of the model should not be limited to quality improvement interventions.

Study findings heighten the need for multilevel efforts to improve the likelihood of success of these processes, considering the complexity and particularities of safety net organization like CHCs. PCMH standards established by current accrediting organizations serve as general guidelines for the development of patient care and quality improvement efforts identified as evidence-based best practices. However, PCMH standards do not provide health organizations with guidance on how to effectively implement the recommended practices. PCMH standards should also include guidelines regarding organizational structure, management, staff needed to establish the model Current PCMH standards do not consider these organizational factors. Furthermore, even when they are highly encouraged by HRSA to obtain PCMH recognition, HRSA-funded CHCs do not currently receive any organizational support or guidance from this agency to effectively implement the model. In fact, given its role in 
the direction and scope of the work of CHCs, HRSA should take a stand regarding the implementation of the PCMH model. This role should not only be delegated to accrediting agencies. And when the latter, it is suggested that all accrediting agencies conform to the same standards of implementation, monitoring and evaluation. The implementation of the PCMH, for example, could also be part of the focus of HRSA operational site visits at CHCs. This would further encourage compliance with the model and the standards of the model. It has so far proven effective with the establishment of clinical and financial performance measures and the requirement to report these measures through the Uniform Data System. Focus on HRSA performance measures has kept CHCs engaged in strategies to improve measures in those areas prioritized by HRSA.

Organizational-level strategies can also foster the growth and strengthen the capacity of key patient care areas like nursing and health education, both critical to chronic disease management. Nurses give continuity to the patient care process and are a central piece of the chronic care strategy. Allied health professionals such as health educators and nutritionists also nurture these health centers' approach to health promotion, an essential component of the work of these organizations, both at the health center level and the community level. PCMH implementation must ensure a solid shared vision, strong management, commitment from all levels of the organization, and an effective organizational infrastructure that leads to improved patient care performance and, ultimately, improved disease outcomes. 


\section{REFERENCES}

Accreditation Association for Ambulatory Health Care, Inc. (AAAHC) (2013). Medical Home on-site certification handbook. Retrieved from http://www.aaahc.org/ Global/Handbooks/AAAHC_MH\%20OSC\%20HB13_Final.pdf.

Ackroyd, S. A., \& Wexler, D. J. (2014). Effectiveness of diabetes interventions in the patient-centered medical home. Current Diabetes Reports, 14(3), 1-9.

Agency for Healthcare Research and Quality (2013). Contextual factors: The importance of considering and reporting on context in research on the patient-centered medical home. Rockville, MD: Agency for Healthcare Research and Quality.

American Diabetes Association (2013). Statistics about diabetes. Retrieved from http://www.diabetes.org/diabetes-basics/statistics/.

American Heart Association (2016). Learn how HBP can harm your health. Retrieved from http://www.heart.org/HEARTORG/Conditions/HighBloodPressure/ WhyBloodPressureMatters/Why-Blood-PressureMatters_UCM_002051_Article.jsp\#.

Anderson, D. R., \& Olayiwola, J. N. (2012). Community health centers and the patientcentered medical home: challenges and opportunities to reduce health care disparities in America. Journal of Health Care for the Poor and Underserved, 23(3), 949-957.

Barr, V. J., Robinson, S., Marin-Link, B., Underhill, L., Dotts, A., Ravensdale, D., \& Salivaras, S. (2003). The Expanded Chronic Care Model: an integration of concepts and strategies from population health promotion and the Chronic Care Model. Healthcare Quarterly, 7(1), 73-82.

Beacham, A.O., Kinman, C., Harris, J.G., \& Masters, K.S. (2012). The Patient-Centered Medical Home: Unprecedented workforce growth potential for professional psychology. Professional Psychology: Research and Practice,43 (1), 17-23.

Bodenheimer, T., Wagner, E.H., \& Grumbach, K. (2002). Improving primary care for patients with chronic illness. Journal of the American Medical Association, 288 (14), 1775-1779.

Bojadzievski T. \& Gabbay, R.A. (2011). Patient-centered medical home and diabetes. Diabetes Care, 34, 1047-1053. 
Brown, T. M., Parmar, G., Durant, R. W., Halanych, J. H., Hovater, M. M., Muntner, P., ... \& Safford, M. M. (2011). Health professional shortage areas, insurance status, and cardiovascular disease prevention in the reasons for geographic and racial differences in stroke (REGARDS) study. Journal of Health Care for the Poor and Underserved, 22(4), 1179.

Buttorff, C., Ruder, T., \& Bauman, M. (2017). Multiple chronic conditions in the United States. Santa Monica (CA): RAND Corporation.

Centers for Disease Control and Prevention (2017). National diabetes statistics report: Estimates of diabetes and its burden in the United States, 2017. Atlanta, GA: U.S. Department of Health and Human Services.

Chow, E. A., Foster, H., Gonzalez, V., \& McIver, L. (2012). The disparate impact of diabetes on racial/ethnic minority populations. Clinical Diabetes, 30(3), 130-133.

Cohen, D. \& Crabtree B. (2006). Theory-based or theoretical sampling. In Qualitative Research Guidelines Project. Retrieved from http://www.qualres.org/HomeTheo3806.html.

Cook, N., Hollar, L., Isaac, E., Paul, L., Amofah, A., \& Shi, L. (2015). Patient experience in health center medical homes. Journal of Community Health, 40(6), 1155-1164.

Creswell, J.W. (2013). Qualitative inquiry \& research design: Choosing among five approaches (third edition). Thousand Oaks, CA: Sage Publications.

Damschroder L., Peikes D., \& Petersen D. (2013). Using implementation research to guide adaptation, implementation, and dissemination of Patient-Centered Medical Home models. Rockville, MD: Agency for Healthcare Research and Quality.

Davy, C., Bleasel, J., Liu, H., Tchan, M., Ponniah, S., \& Brown, A. (2015). Effectiveness of chronic care models: opportunities for improving healthcare practice and health outcomes: a systematic review. BMC Health Services Research, 15(1), 194.

Denzin, N. K., \& Lincoln, Y. S. (2008). Strategies of qualitative inquiry (2nd ed.). Thousand Oaks, CA: Sage Publications.

Department of Health and Human Services (2014). About Healthy People 2020. Retrieved from http://www.healthypeople.gov/2020/About-Healthy-People. 
DePuccio, M. J., \& Hoff, T. J. (2014). Medical Home Interventions and Quality Outcomes for Older Adults: A Systematic Review. Quality Management in Healthcare, 23(4), 226-239.

Dobbins, J. M., Peiper, N., Jones, E., Clayton, R., Peterson, L. E., \& Phillips Jr, R. L. (2018). Patient-Centered Medical Home recognition and diabetes control among health centers: Exploring the role of enabling services. Population Health Management, 21(1), 6-12.

Elissen, A., Nolte, E., Hinrichs, S., Conklin, A., Adams, J., Cadier, B., ... \& Frølich, A. (2014). Evaluating chronic disease management in real-world settings in six European countries: Lessons from the collaborative DISMEVAL project. International Journal of Care Coordination, 17(1-2), 25-37.

Forbes, A., \& While, A. (2009). The nursing contribution to chronic disease management: a discussion paper. International Journal of Nursing Studies, 46(1), 120-131.

Geertz, C. (1973). The interpretation of cultures: selected essays. New York: Basic Books.

Glasgow, R. E., Funnell, M. M., Bonomi, A. E., Davis, C., Beckham, V., \& Wagner, E. H. (2002). Self-management aspects of the improving chronic illness care breakthrough series: implementation with diabetes and heart failure teams. Annals of Behavioral Medicine, 24(2), 80-87.

Glasgow, R.E. \& Stange, K.C. (2014). Health system models. In Riekert, K. A., Ockene, J. K., \& Pbert, L. (Eds.). The Handbook of Health Behavior Change (47-65). New York, NY: Springer Publishing Company.

Goldman, R. E., Parker, D. R., Brown, J., Walker, J., Eaton, C. B., \& Borkan, J. M. (2015). Recommendations for a mixed methods approach to evaluating the Patient-Centered Medical Home. The Annals of Family Medicine, 13(2), 168-175.

Gray, D. E. (2013). Doing research in the real world. Chicago, IL: Sage.

Green, E. P., Wendland, J., Carver, M. C., Rinker, C. H., \& Mun, S. K. (2012). Lessons learned from implementing the patient-centered medical home. International Journal of Telemedicine and Applications, 2012 (6), 1-8.

Health Resources and Services Administration (HRSA) (2017). 2016 Health Center Program factsheet. Retrieved from https://www.bphc.hrsa.gov/about/ healthcenterfactsheet.pdf. 
Health Resources and Services Administration (HRSA) (2018a). 2017 national health center data. Retrieved from http://bphc.hrsa.gov/uds/datacenter.aspx.

Health Resources and Services Administration (HRSA) (2018b). Health Center Quality Improvement FY 2018 Grant Awards. Retrieved from https://bphc.hrsa.gov/ programopportunities/fundingopportunities/qualityimprovement/index.html.

Health Resources and Services Administration (HRSA) (2018c). 2017 Health Center Program grantee data. Retrieved from https://bphc.hrsa.gov/uds/ datacenter.aspx? $\mathrm{q}=\mathrm{d}$.

Health Resources and Services Administration (HRSA) (2018d). Health Center Program compliance manual. Retrieved from https://bphc.hrsa.gov/programrequirements/ compliancemanual/ index.html.

Healthy People 2020 (2017). Diabetes. Washington, DC: U.S. Department of Health and Human Services, Office of Disease Prevention and Health Promotion. Retrieved from https://www.healthypeople.gov/2020/topics-objectives/topic/diabetes.

Hoff, T. (2010). Practice under pressure: Primary care physicians and their medicine in the twenty-first century. New Brunswick, NJ: Rutgers University Press.

Hoff, T., Weller, W., \& DePuccio, M. (2012). The Patient-Centered Medical Home: A review of recent research. Medical Care Research and Review,69(6), 619-644.

Holtrop, J. S., \& Jordan, T. R. (2010). The patient-centered medical home and why it matters to health educators. Health Promotion Practice, 11(5), 622-628.

Institute of Medicine (2012). Living well with chronic illness: A call for public health action. Washington, DC: The National Academies Press.

Jackson, G. L., Powers, B. J., Chatterjee, R., Bettger, J. P., Kemper, A. R., Hasselblad, V., ... \& Williams, J. W. (2013). The patient-centered medical home: a systematic review. Annals of Internal Medicine, 158(3), 169-178.

James, W.L., Matthews, K.C., Albrecht, P.A., Fox, S., \& Church, A. (2017). Evidence of a Patient-Centered Medical Home (PCMH) improving the health of chronically ill patients in the Mississippi Delta. Journal of Preventive Medicine and Care, 1(4), 8-16.

Kennedy, A., Rogers, A., \& Bower, P. (2007). Support for self-care for patients with chronic disease. British Medical Journal, 335(7627), 968. 
Liang, S., Kegler, M. C., Cotter, M., Phillips, E., Beasley, D., Hermstad, A., ... \& Riehman, K. (2016). Integrating evidence-based practices for increasing cancer screenings in safety net health systems: a multiple case study using the Consolidated Framework for Implementation Research. Implementation Science, 11(1), 109.

Lieberthal, R. D., Karagiannis, T., Bilheimer, E., Verma, M., Payton, C., Sarfaty, M., \& Valko, G. (2017). Exploring variation in transformation of primary care practices to patient-centered medical homes: a mixed methods approach. Population Health Management, 20(5), 411-418.

Luxford, K., Safran, D. G., \& Delbanco, T. (2011). Promoting patient-centered care: a qualitative study of facilitators and barriers in healthcare organizations with a reputation for improving the patient experience. International Journal for Quality in Health Care, 23(5), 510-515.

Martsolf, G.R., Alexander, J.A., Shi, Y., Casalino, L.P., Rittenhouse, D.R., Scanlon, D.P., Shortell, S.M. (2012). The patient-centered medical home and patient experience. Health Services Research, 47(6), 2273-2295.

Milani, R. V., \& Lavie, C. J. (2015). Health care 2020: Reengineering health care delivery to combat chronic disease. The American Journal of Medicine, 128(4), 337-343.

Miller, D., \& Baumgartner, E. T. (2016). Lessons from the Community-Centered Health Home Demonstration Project: Patient-Centered Medical Homes can improve health conditions in their surrounding communities. Preventing Chronic Disease, 13, E102. Retrieved from https://www.ncbi.nlm.nih.gov/pmc/articles/ PMC4975202/pdf/PCD-13-E102.pdf.

Miller, W. L., Crabtree, B. F., McDaniel, R., \& Stange, K. C. (1998). Understanding change in primary care practice using complexity theory. Journal of Family Practice, 46(5), 369-377.

Miller-Day, M., Applequist, J., Zabokrtsky, K., Dalton, A., Kellom, K., Gabbay, R., \& Cronholm, P.F. (2017). A tale of four practices: A comparative analysis of high and low performing patient-centered medical homes. Journal of Health Organization and Management, 31(6), 630-646.

Morgan, T. O., Everett, D. L., \& Dunlop, A. L. (2014). How do interventions that exemplify the joint principles of the Patient Centered Medical Home affect hemoglobin A1C in patients with diabetes: A review. Health Services Research and Managerial Epidemiology, 1, 2333392814556153. 
National Association of Community Health Centers (2011). So you want to start a health center? A practical guide for starting a Federally Qualified Health Center. Bethesda, MD: Author.

National Committee for Quality Assurance (NCQA) (2017). NCQA Patient-Centered Medical Home standards and guidelines (2017 edition, version 2). Washington, DC: Author.

National Association of Community Health Centers (NACHC) (2017). Community Health Center chartbook. June 2017. Retrieved from http://www.nachc.org/wpcontent/uploads/2017/06/Chartbook2017.pdf.

Nuño, R., Coleman, K., Bengoa, R., \& Sauto, R. (2012). Integrated care for chronic conditions: The contribution of the ICCC Framework. Health Policy, 105 (2012), $55-64$.

Ottmar, J., Blackburn, B., Phillips Jr, R. L., Peterson, L. E., \& Jaen, C. R. (2015). Family physicians' ability to perform population management is associated with adoption of other aspects of the patient-centered medical home. Population Health Management, 18(2), 72-78.

Patton, M. Q. (2002). Qualitative research and evaluation methods. Thousand Oaks, CA: Sage.

Perez, B., Cummings, L., Schrag, J., Mead, H., \& Jewers, M. (2013). Facilitators and barriers to providing patient-centered chronic disease care to patient populations at risk for health and health care disparities in safety net settings. Retrieved from https://www.pcori.org/assets/2014/01/PCORI-Facilitators-Barriers-ProvidingPatient-Centered-Chronic-Disease-Care-120613.pdf.

Platonova, E. R., Warren-Findlow, J., Saunders, W. J., Hutchison, J. A., \& Coffman, M. J. (2016). Hispanics' satisfaction with free clinic providers: an analysis of patientcentered medical home characteristics. Journal of Community Health, 41(6), 1290-1297.

Price, J. H., Khubchandani, J., McKinney, M., \& Braun, R. (2013). Racial/ethnic disparities in chronic diseases of youths and access to health care in the United States. BioMed Research International, 2013.

Quinn, M. T., Gunter, K. E., Nocon, R. S., Lewis, S. E., Vable, A. M., Tang, H., ... \& Chin, M. H. (2013). Undergoing transformation to the patient centered medical 
home in safety net health centers: perspectives from the front lines. Ethnicity \& Disease, 23(3), 356-362.

Rios, E. (May 15, 2017). The looming healthcare crisis on top of Puerto Rico's economic woes. The Hill. Retrieved from http://thehill.com/blogs/punditsblog/healthcare/333364-theres-a-looming-healthcare-crisis-on-top-of-puertoricos.

Rivo, J., Page, T. F., Arrieta, A., Amofah, S. A., McCann, S., Kassaye, H., ... \& Williams, M. L. (2016). The impact of comprehensive pre-visit preparation on patient engagement and quality of care in a population of underserved patients with diabetes: evidence from the care management medical home center model. Population Health Management, 19(3), 171-177.

Rosenbaum, S., Shin, P., Jones, E., \& Tolbert, J. (2010). Community Health Centers: Opportunities and challenges of health reform. Washington, DC: The Henry J. Kaiser Family Foundation.

Schadewaldt, V., McInnes, E., Hiller, J. E., \& Gardner, A. (2014). Investigating characteristics of collaboration between nurse practitioners and medical practitioners in primary healthcare: a mixed methods multiple case study protocol. Journal of Advanced Nursing, 70(5), 1184-1193.

Schulman-Green, D., Jaser, S. , Martin, F., Alonzo, A., Grey, M., McCorkle, R., Redeker, N.S., Reynolds, N., \& Whittemore, R. (2012). Processes of self-management in chronic illness. Journal of Nursing Scholarship, 44(2), 136-144.

Setodji, C. M., Quigley, D. D., Elliott, M. N., Burkhart, Q., Hochman, M. E., Chen, A. Y., \& Hays, R. D. (2017). Patient experiences with care differ with chronic care management in a Federally Qualified Community Health Center. Population Health Management, 20(6), 442-448.

Shi, L., Lock, D. C., Lee, D. C., Lebrun-Harris, L. A., Chin, M. H., Chidambaran, P., ... \& Sripipatana, A. (2015). Patient-Centered Medical Home capability and clinical performance in HRSA-supported health centers. Medical Care, 53(5), 389-395.

Shin, P., Ku, L. C., Jones, E., Finnegan, B., \& Rosenbaum, S. J. (2009). Financing community health centers as patient and community-centered medical homes: a primer. Washington, DC: The George Washington University. 
Shin, P., Sharac, J., Luis, M.N., \& Rosenbaum, S. (2015). Puerto Rico's community health centers in a time of crisis. RCHN Community Health Foundation Research Collaborative (Policy Research Brief \# 43). Retrieved from https://publichealth.gwu.edu/sites/default/files/downloads/GGRCHN/Policy\%20R esearch\%20Brief\%2043.pdf.

Shippee, N. D., Finch, M., \& Wholey, D. (2017). Using statewide data on health care quality to assess the effect of a Patient-Centered Medical Home initiative on quality of care. Population Health Management, 21(2), 148-154.

Smith, L. J., Johnson-Lawrence, V., Andrews, M., \& Parker, S. (2017). Opportunity for interprofessional collaborative care-findings from a sample of federally qualified health center patients in the Midwest. Public Health, 151, 131-136.

Stake, R. E. (1995). The art of case study research. Thousand Oaks, CA: Sage.

Taylor, J. (2004). Fundamentals of community health centers. Retrieved from http://www.nhpf.org/library/background-papers/BP_CHC_08-31-04.pdf.

Timbie, J. W., Hussey, P. S., Setodji, C. M., Kress, A., Malsberger, R., Lavelle, T. A., ... \& Kahn, K. L. (2017a). Association between Patient-Centered Medical Home capabilities and outcomes for Medicare beneficiaries seeking care from Federally Qualified Health Centers. Journal of General Internal Medicine, 32(9), 997-1004.

Timbie, J. W., Setodji, C. M., Kress, A., Lavelle, T. A., Friedberg, M. W., Mendel, P. J., ... \& Kommareddi, M. (2017b). Implementation of Medical Homes in Federally Qualified Health Centers. New England Journal of Medicine, 377(3), 246-256.

Towne, S. D., Bolin, J., Ferdinand, A., Nicklett, E. J., Smith, M. L., \& Ory, M. G. (2017). Assessing diabetes and factors associated with foregoing medical care among persons with diabetes: Disparities facing American Indian/Alaska native, black, Hispanic, low income, and southern adults in the US (2011-2015). International Journal of Environmental Research and Public Health, 14(5), 464.

Tu, S. P., Young, V. M., Coombs, L. J., Williams, R. S., Kegler, M. C., Kimura, A. T., ... \& Fernandez, M. E. (2015). Practice adaptive reserve and colorectal cancer screening best practices at community health center clinics in 7 states. Cancer, 121(8), 1241-1248. 
U.S. Centers for Medicare \& Medicaid Services (2018). Physician compare. Retrieved from https://www.medicare.gov/physiciancompare/

Wagner, E.H. (1998) Chronic disease management: what will it take to improve care for chronic illness? Effective Clinical Practice: ECP, 1998 (1), 2-4.

Wagner, E. H. (2000). The role of patient care teams in chronic disease management. Bmj, 320(7234), 569-572.

Wagner, E. H., Austin, B. T., Davis, C., Hindmarsh, M., Schaefer, J., \& Bonomi, A. (2001). Improving chronic illness care: translating evidence into action. Health Affairs, 20(6), 64-78.

Wagner, E. H., Coleman, K., Reid, R. J., Phillips, K., \& Sugarman, J. R. (2012). Guiding transformation: How medical practices can become patient-centered medical homes. New York, NY: The Commonwealth Fund. Retrieved from https://www.commonwealthfund.org/sites/default/files/documents/__media_files _publications_fund_report_2012_feb_1582_wagner_guiding_transformation_pati entcentered_med_home_v2.pdf.

Yin, R.K. (2003). Case study research: design and methods ( $3^{\text {rd }}$ ed). Thousand Oaks, CA: Sage.

Zhang, Y. \& Wildemuth, B. M. (2009). Qualitative analysis of content. In B. Wildemuth (Ed.), Applications of Social Research Methods to Questions in Information and Library Science, 308-319. Westport, CT: Libraries Unlimited.

Zwar, N., Harris, M., Griffiths, R., Roland, M., Dennis, S., Powell Davies, G., \& Hasan, I. (2006). A systematic review of chronic disease management. Retrieved from https://openresearch-repository.anu.edu.au/bitstream/1885/119226/3/ final_25_zwar_pdf_85791.pdf. 


\section{APPENDICES}


Appendix A: IRB Approval Letter

\section{FLORIDA}

INTERNATIONAL

UNIVERSITY

\section{MEMORANDUM}

To: Dr. Elena Bastida, Principal Investigator

CC: $\quad$ File

From: Eliza Gomez, M.Ed., Coordinator, Research Integrity $C t$

Date: Janurary 12,2017

Protocol Title: $\quad$ "Contextual Factors in Patient-Centered Medical Home Implementation and Chronic Disease Management in HRSA-Funded Community Health Centers"

The Florida International University Office of Research Integrity has reviewed your research study for the use of human subjects and deemed it Exempt via the Exempt Review process.

IRB Protocol Exemption \#: IRB-17-0010 IRB Exemption Date: 01/12/17

TOPAZ Reference \#: $\quad 105419$

As a requirement of $I R B$ Exemption you are required to:

1) Submit an Event Form and provide immediate notification of:

- Any additions or changes in the procedures involving human subjects.

- Every serious or unusual or unanticipated adverse event as well as problems with the rights or welfare of the human subjects.

2) Submit a Project Completion Report Form when the study is finished or discontinued.

Special N/A

Conditions:

For further information, you may visit the $\mathbb{R} B$ website at http://research.fiu.edu/irb. 


\title{
Appendix B: Informational Letter for Participating Health Centers
}

\author{
INFORMATIONAL LETTER \\ Organizational factors in Patient-Centered Medical Home implementation and chronic \\ disease management in HRSA-funded community health centers
}

Hello, my name is Willmarie Latorre. Your health center has agreed to participate in a research study about organizational factors that affect the implementation of the Patient-Centered Medical Home model and chronic disease management efforts at community health centers funded by HRSA.

The purpose of the study is to explore and describe organizational-level factors that contribute to or hinder $\mathrm{PCMH}$ implementation and improvements in chronic disease management among $\mathrm{CHCs}$. If you agree, you will be part of an individual or group interview process with other health center team members. Participation in this process could take up to an hour.

During the interview session, the researcher will guide an interactive question-answer session about organizational contextual factors affecting PCMH implementation and chronic disease management efforts.

There are no foreseeable risks or benefits to you for participating in this study. Your health center will have access to findings, conclusions, and an organizational PCMH implementation model that will be developed to support PCMH implementation and chronic disease management among HRSA-funded CHCs. Findings can be used to improve the implementation of the model and chronic disease management efforts. Supporting PCMH implementation and chronic disease management could help health centers nationwide advance their efforts to promote health and enhance quality of life of patients affected by chronic diseases.

There is no cost or payment to you or your health center. If you have questions while taking part, please stop me and ask.

The session will be audio-recorded solely to facilitate transcription and analysis of responses. Only the researcher will have access to this audio. As findings are documented, analyzed, and reported, your responses will remain anonymous.

If you have questions, you may contact me at (786) 395-8110 or wlato001@fiu.edu. If you would like to talk with someone about your rights of being a subject in this research study or about ethical issues with this research study, you may contact the FIU Office of Research Integrity by phone at 305-348-2494 or by email at ori@fiu.edu.

Your participation in this research is voluntary, and you will not be penalized or lose benefits if you refuse to participate or decide to stop. You may keep a copy of this form for your records. 
Appendix C: Observation Tool 
Organizational factors and dynamics that drive successful PCMH implementation and improved chronic disease management

FIELD OBSERVATION CHECKLIST AND RECORD

INSTRUCTIONS FOR OBSERVER: Complete one Field Observation Checklist for each health center visited. Use the spaces provided to note observations

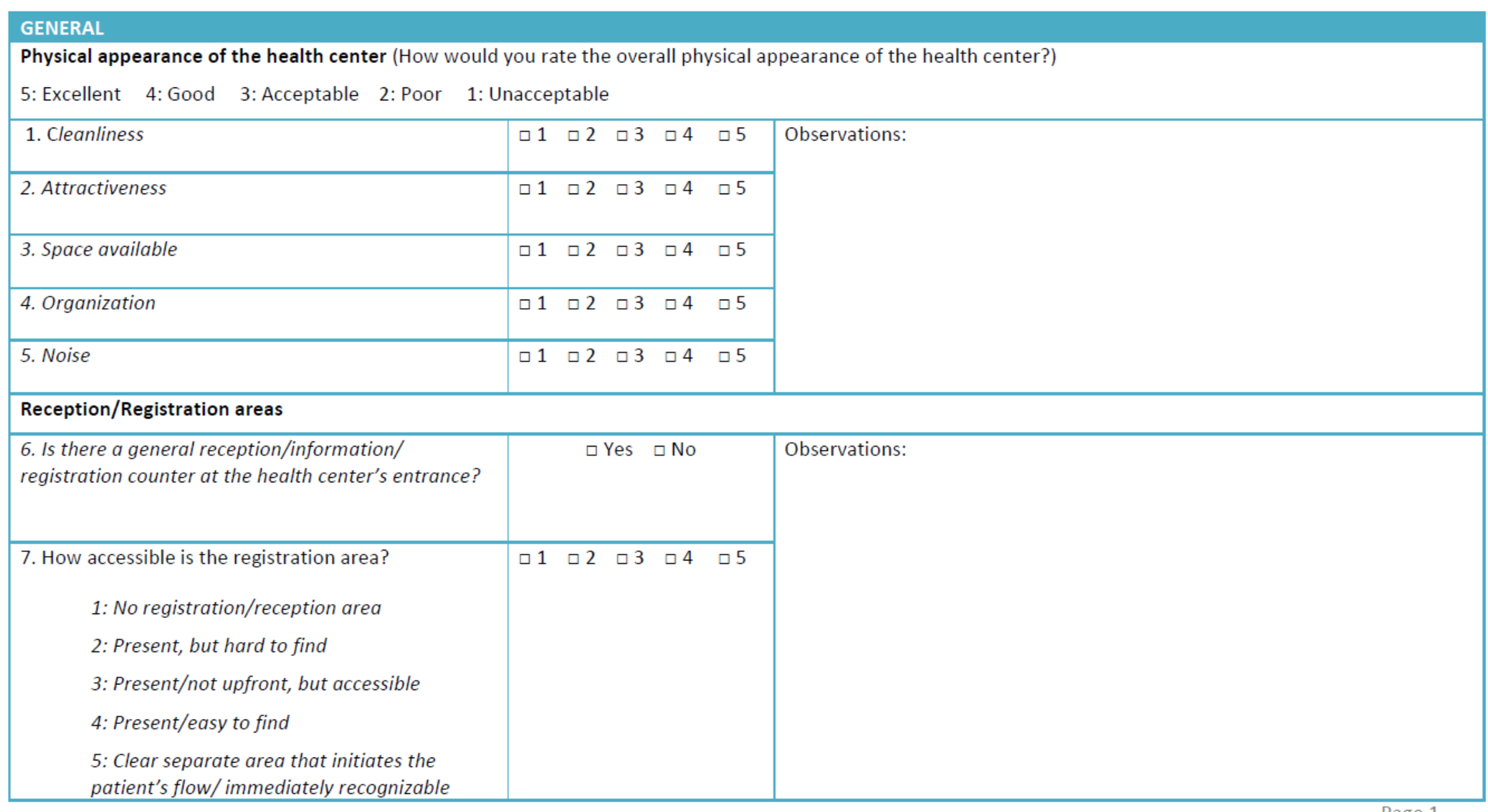


Organizational factors in HRSA-funded CHCs

$\begin{array}{ll}\text { Date: } & \text { Start time: } \\ \text { Health Center ID: } & \text { End time: }\end{array}$

\begin{tabular}{|c|c|c|}
\hline \multicolumn{3}{|l|}{ Patient waiting area } \\
\hline $\begin{array}{l}\text { 8. Characteristics of the waiting area (Check all that } \\
\text { apply) }\end{array}$ & $\begin{array}{l}\square \text { One general waiting area } \\
\square \text { Separate waiting areas } \\
\text { for each step in the process } \\
\square \text { Clean/spaced } \\
\square \text { Print educational } \\
\text { resources available (e.g., } \\
\text { print materials) } \\
\square \text { TV screens with } \\
\text { educational content } \\
\square \text { Educational kiosks } \\
\square \text { Educational posters or } \\
\text { digital displays } \\
\square \text { Patient events taking } \\
\text { place in waiting area } \\
\square \text { Other (specify) }\end{array}$ & Observations: \\
\hline $\begin{array}{l}\text { 9. Patient load in the waiting room } \\
\text { 1: Overcrowded } \\
\text { 2: Crowded } \\
\text { 3: Moderate } \\
\text { 4: Light } \\
\text { 5: Very light }\end{array}$ & $\square 1 \quad \square 2 \quad \square 3 \quad \square 4 \quad \square 5$ & \\
\hline
\end{tabular}




\section{Patient flow}

Health Center ID:

End time:

\begin{tabular}{|c|c|c|}
\hline $\begin{array}{l}\text { 10. Estimated average patient waiting time in } \\
\text { waiting area }\end{array}$ & $\begin{array}{l}\square \text { Less than } 15 \text { minutes } \\
\square 15 \text { to } 30 \text { minutes } \\
\square 31 \text { to } 45 \text { minutes } \\
\square 46 \text { to } 60 \text { minutes } \\
\square \text { More than } 1 \text { hour }\end{array}$ & Observations: \\
\hline $\begin{array}{l}\text { 11. Cycle time - How long does it take patients to } \\
\text { move from one area/process to the other, until the } \\
\text { patient finishes the cycle? (e.g., from moment patient } \\
\text { is called to vitals to provider encounter to checkout) }\end{array}$ & $\begin{array}{l}\square \text { Less than } 15 \text { minutes } \\
\square 15 \text { to } 30 \text { minutes } \\
\square 31 \text { to } 45 \text { minutes } \\
\square 46 \text { to } 60 \text { minutes } \\
\square \text { More than } 1 \text { hour }\end{array}$ & \\
\hline
\end{tabular}

Announcements (written, visual, or electronic) available on site

\begin{tabular}{l|l|}
\hline $\begin{array}{l}\text { 12. Availability of announcements for patients (Check } \\
\text { all that apply) }\end{array}$ & $\begin{array}{l}\square \text { Print/ Written } \\
\square \text { Print/Visual } \\
\square \text { Electronic/ digital }\end{array}$ \\
\hline $\begin{array}{l}\text { 13. Areas where these are located } \\
\square \text { Entrance } \\
\square \text { Registration/ reception } \\
\square \text { Waiting area } \\
\square \text { Hallways } \\
\square \text { Other (specify) }\end{array}$ & $\square$ English $\square$ Spanish \\
& $\square$ Portuguese $\square$ Creole \\
$\begin{array}{l}\text { 14. Languages (Check all that apply) } \\
\square \text { Other (specify) }\end{array}$ \\
$\begin{array}{l}\text { 15. Announcements/communications to educate } \\
\text { patients on health-related issues (Describe) }\end{array}$ & $\square$ Yes $\square$ No
\end{tabular}

Observations:

patients on health-related issues (Describe) 
Organizational factors in HRSA-funded CHCs

\section{Announcements (written, visual, or electronic) available on site}

\begin{tabular}{|c|c|c|}
\hline 16. Contents, topics, or issues & Describe & Observations: \\
\hline $\begin{array}{l}\text { 17. Level of readability (make first hand observations } \\
\text { and take samples to analyze using the Flesch-Kincaid } \\
\text { Grade Level Readbility Scale and the Fry Readability } \\
\text { Graph) }\end{array}$ & $\begin{array}{lll}\square<6^{\text {th }} & \square 6^{\text {th }} & \square 7^{\text {th }} \\
\square 8^{\text {th }} & \square 9^{\text {th }} & \square>9^{\text {th }}\end{array}$ & \\
\hline $\begin{array}{l}\text { 18. Content appropriateness for the patient } \\
\text { population (Describe) }\end{array}$ & $\begin{array}{l}\square \text { Use of complex medical } \\
\text { terminology } \\
\square \text { Use of several concepts } \\
\text { per paragraph or section } \\
\square \text { Illustrations use to } \\
\text { facilitate comprehension } \\
\square \text { Irrelevant/unrelated } \\
\text { illustrations } \\
\square \text { Includes advice/ call to } \\
\text { action } \\
\square \text { Other (specify) }\end{array}$ & \\
\hline 19. Cultural sensitivity & $\begin{array}{l}\square \text { Material available in } \\
\text { more than one language } \\
\square \text { Use of culturally } \\
\text { appropriate visuals } \\
\square \text { Respect of cultural } \\
\text { beliefs } \\
\square \text { Respectful discussion of } \\
\text { sensitive issues for } \\
\text { vulnerable groups (LGBT, } \\
\text { farmworkers, homeless, } \\
\text { veterans, elderly) }\end{array}$ & \\
\hline
\end{tabular}




\section{Technology Use}

20. Check all the systems used at the health center

(Document observations regarding the use of these system

systems)

$\square$ Electronic health record

$\square$ Electronic prescribing

$\square$ Other

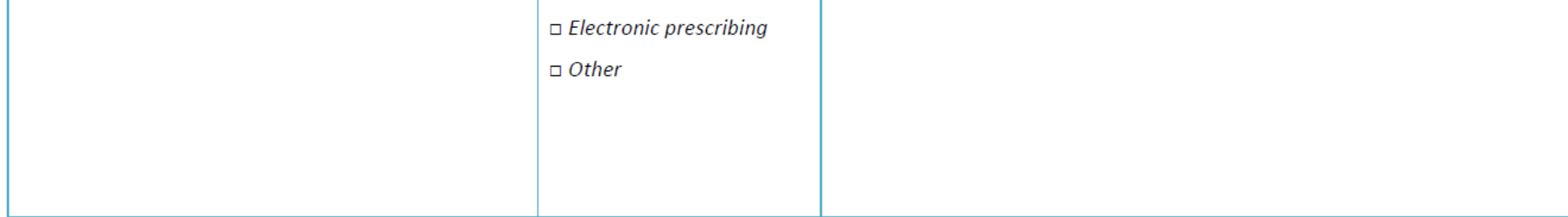

INTERVENTION CHARACTERISTICS (Observation on the implementation of the PCMH model and chronic disease management/support efforts)

\section{COMPREHENSIVE CARE}

21. Comprehensiveness of services provided (physical,

mental/behavioral health care needs, including

prevention and wellness; acute care; and chronic

care)

$\checkmark$ General/Family medicine Observations:

Internal medicine

$\square$ Pediatrics

$\square$ OB/Gyn

$\square$ Preventive screenings

$\square$ Immunization

$\square$ Specialized care

$\square$ Mental/behavioral health

$\square$ Dental

$\square$ Vision

$\square$ Emergency/Urgency care

$\square$ Nutrition

$\square$ Health Education

$\square$ Case management

$\square$ Physical therapy

$\square$ Pharmacy

$\square$ Radiology

- Laboratory

$\square$ Transportation

$\square$ Other 


\section{COMPREHENSIVE CARE}

22. Multidisciplinary team-based care

Rate the level of multidisciplinary team-based care observed

(From 0: None observed to 7: Highly dynamic level of team-based care observed)

23. Multidisciplinary patient-centric team-based care (team is inclusive of patients and accountable to them)

From 0: No interactions with patients observed to $\mathbf{7}$ : highly dynamic interaction between health care team members and patients

$$
\mid
$$

PATIENT-CENTERED CARE (Observations on how the health center provides relationship-based care)

\begin{tabular}{|c|c|c|}
\hline $\begin{array}{l}\text { Rate from 0: not present to } 7 \text { : highly present } \\
\text { 24. Partnerships with patients and their families }\end{array}$ & $\begin{array}{lll}\square 1 & \square 2 & \square 3 \\
\square & \square \\
\square & \square 6 & \square 7\end{array}$ & Observations: \\
\hline $\begin{array}{l}\text { 25. Understanding and respect for patient's needs, } \\
\text { culture, values, and preferences }\end{array}$ & $\begin{array}{lll}\square 1 & \square 2 & \square 3 \quad \square 4 \\
\square 5 & \square 6 & \square 7\end{array}$ & \\
\hline
\end{tabular}




\begin{tabular}{|c|c|c|c|}
\hline 26. Walk-in/same-day patients accepted & $\square$ Yes & $\square$ No & \multirow[t]{6}{*}{ Observations: } \\
\hline 27. Services being provided after $5 \mathrm{pm}$ & $\square$ Yes & $\square$ No & \\
\hline 28. Services provided on weekends & $\square$ Yes & $\square$ No & \\
\hline $\begin{array}{l}\text { 29. Availability of appointments within the next two } \\
\text { weeks }\end{array}$ & $\square$ Yes & $\square$ No & \\
\hline $\begin{array}{l}\text { 30. Announcements (written, visual, or electronic) } \\
\text { indicating availability of access to care or clinical } \\
\text { advice } 24 / 7\end{array}$ & $\square$ Yes & $\square$ No & \\
\hline $\begin{array}{l}\text { 31. Announcements instructing patients what to do to } \\
\text { access care or clinical advice after health center closes } \\
\text { (after-hours) }\end{array}$ & $\square$ Yes & $\square$ No & \\
\hline \multicolumn{4}{|l|}{ PATIENT SUPPORT/ ENABLING SERVICES } \\
\hline $\begin{array}{l}\text { Transportation } \\
\text { 32. Are patients being offered transportation } \\
\text { services? }\end{array}$ & $\square$ Yes & $\square$ No & \multirow[t]{2}{*}{ Observations: } \\
\hline $\begin{array}{l}\text { 33. Any health center van(s) to transport patients to } \\
\text { and from the health center? }\end{array}$ & $\square$ Yes & $\square$ No & \\
\hline
\end{tabular}




\section{PATIENT SUPPORT/ ENABLING SERVICES}

\begin{tabular}{|c|c|c|c|}
\hline $\begin{array}{l}\text { Health care coverage assistance } \\
\text { 34. Availability of health care coverage assistance or } \\
\text { support staff }\end{array}$ & $\square$ Yes & $\square$ No & \multirow[t]{4}{*}{ Observations: } \\
\hline $\begin{array}{l}\text { 35. Announcements indicating availability of this } \\
\text { service }\end{array}$ & $\square$ Yes & $\square$ No & \\
\hline 36. Any patient being offered the service & $\square$ Yes & $\square$ No & \\
\hline $\begin{array}{l}\text { 37. Any patient indicating that he/she has received } \\
\text { the service }\end{array}$ & $\square$ Yes & $\square$ No & \\
\hline $\begin{array}{l}\text { Translation/interpretation services (Other } \\
\text { languages, sign language) } \\
\text { 38. Are translators available when needed? }\end{array}$ & $\square$ Yes & $\square$ No & \multirow[t]{3}{*}{ Observations: } \\
\hline $\begin{array}{l}\text { 39. Does health center staff speak to the patient in } \\
\text { the language of preference? }\end{array}$ & $\square$ Yes & $\square$ No & \\
\hline $\begin{array}{l}\text { 40. Are messages/announcements available in other } \\
\text { languages? }\end{array}$ & $\square$ Yes & $\square$ No & \\
\hline $\begin{array}{l}\text { Other services to remove barriers to care } \\
\text { 41. Financial assistance/counseling }\end{array}$ & $\square$ Yes & $\square$ No & \multirow[t]{4}{*}{ Observations: } \\
\hline 42. Availability of sliding fee discounts & $\square$ Yes & $\square$ No & \\
\hline 43. Case management & $\square$ Yes & $\square$ No & \\
\hline 44. Care coordination & $\square$ Yes & $\square$ No & \\
\hline
\end{tabular}


Organizational factors in HRSA-funded CHCs

\section{STRATEGIC PARTNERSHIPS}

\begin{tabular}{|c|c|c|c|}
\hline $\begin{array}{l}\text { 45. Any collaboration taking place at the community } \\
\text { level (with community organizations, community } \\
\text { members/leaders) }\end{array}$ & $\square$ Yes & $\square$ No & Observations: \\
\hline $\begin{array}{l}\text { 46. Any collaboration taking place with providers } \\
\text { outside the health center (health care system level) }\end{array}$ & $\square$ Yes & $\square$ No & \\
\hline \multicolumn{4}{|c|}{ GUIDANCE AND LEADERSHIP Any observations on staff or provider champions (leaders) facilitating PCMH initiatives } \\
\hline 47. Any particular leadership behaviors noticed? & $\square$ Yes & $\square$ No & Observations: \\
\hline 48. Are you able to observe any outstanding roles? & $\square$ Yes & $\square$ No & \\
\hline
\end{tabular}




\begin{tabular}{|c|c|c|}
\hline PATIENT PARTICIPATION & & \\
\hline $\begin{array}{l}\text { 49. Select and describe observations regarding } \\
\text { patients participating in PCMH activities or chronic } \\
\text { disease management efforts? (Check all that apply) }\end{array}$ & $\begin{array}{l}\square \text { Community events } \\
\square \text { Group patient education } \\
\square \text { Support groups } \\
\square \text { Health fair } \\
\square \text { Patient orientations/ } \\
\text { testimonials } \\
\square \text { Physical activity } \\
\square \text { Patient advisory board } \\
\square \text { Evidence of patient } \\
\text { participation in quality } \\
\text { improvement team } \\
\square \text { Participation in surveys } \\
\square \text { Patient "walk-through" } \\
\square \text { Peer-to-peer surveys } \\
\square \text { Other }\end{array}$ & Observations: \\
\hline $\begin{array}{l}\text { COMMUNICATION } \\
\text { 50. Communication strategies or events related to the } \\
\text { PCMH model and chronic disease management/ } \\
\text { support efforts }\end{array}$ & $\begin{array}{l}\square \text { Distribution of report on } \\
\text { PCMH results } \\
\square \text { Bulletin board/digital } \\
\text { display with charts or } \\
\text { announcements on the } \\
\text { PCMH } \\
\square \text { News release about the } \\
\text { PCMH initiative } \\
\square \text { Announcements on } \\
\text { chronic disease } \\
\text { management efforts } \\
\square \text { Other }\end{array}$ & Observations: \\
\hline
\end{tabular}




\section{INNER SETTING}

\section{STRUCTURAL CHARACTERISTICS}

51. Observation regarding the size of the clinic staff

$\square$ Could not tell

$\checkmark$ Small (10 or less)

$\square$ Medium (11-20)

$\square$ Large (More than 20)

52. Centralization of decision-making

$\square 1 \quad \square 2 \quad \square 3 \quad \square 4$

From 1: Highly centralized at the top level to

$\square 5 \quad \square 6 \quad \square 7$

7: De-centralized (delegation of decision-making powers and flexible processes)

\begin{tabular}{|l|l|}
\hline 53. Size of facilities & $\begin{array}{l}\square \text { Small } \\
\square \text { Medium } \\
\square \text { Large }\end{array}$ \\
\hline $\begin{array}{l}\text { 54. Is each functional or service area in the health } \\
\text { center clearly delimited? }\end{array}$ & $\square$ Yes $\square$ No \\
\hline $\begin{array}{l}\text { 55. Does each area have a clearly designated staff } \\
\text { member? }\end{array}$ & $\square$ Yes $\square$ No \\
\hline $\begin{array}{l}\text { 56. Does each area/unit have a manager/supervisor? } \\
\end{array}$ & $\square$ Yes $\square$ No \\
\hline
\end{tabular}




\section{STRUCTURAL CHARACTERISTICS}

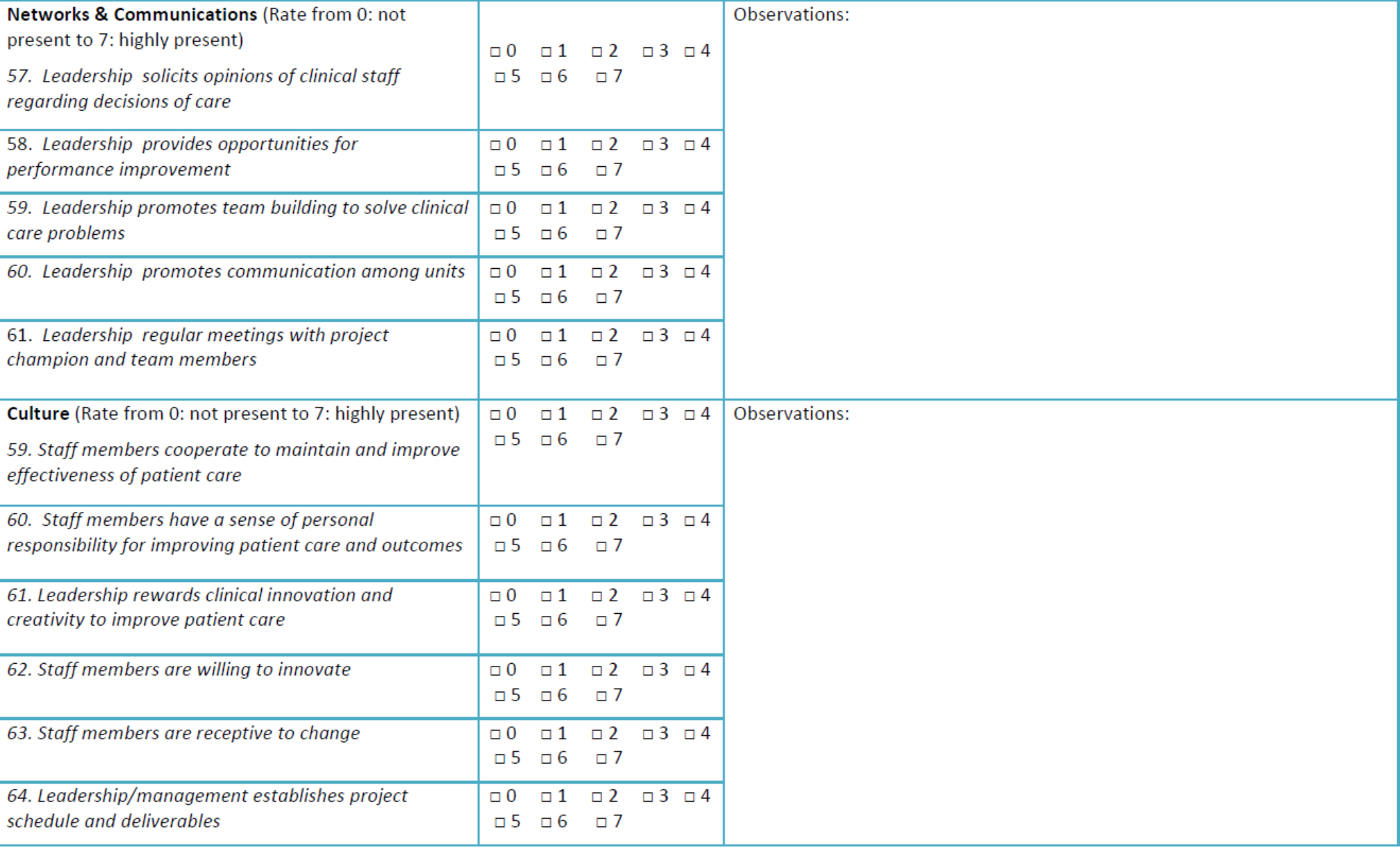


CHARACTERISTICS OF INDIVIDUALS

\begin{tabular}{|c|c|c|c|}
\hline $\begin{array}{l}\text { Based on your observations, rate from 1: Lowest to } 7 \text { : } \\
\text { Highest the following aspects regarding the health } \\
\text { center staff } \\
\text { 77. Team cohesiveness. }\end{array}$ & $\begin{array}{l}\square 1 \\
\square 5\end{array}$ & $\begin{array}{l}\square 2 \\
\square 6\end{array}$ & $\begin{array}{l}\square 3 \square 4 \\
\square 7\end{array}$ \\
\hline 78. Staff empowerment with regards to their roles. & $\begin{array}{l}\square 1 \\
\square 5\end{array}$ & $\begin{array}{l}\square 2 \\
\square 6\end{array}$ & $\begin{array}{l}\square 3 \square 4 \\
\square 7\end{array}$ \\
\hline 79. Staff's level of interest in the patient care process. & $\begin{array}{l}\square 1 \\
\square 5\end{array}$ & $\begin{array}{l}\square 2 \\
\square 6\end{array}$ & $\begin{array}{l}\square 3 \square 4 \\
\square 7\end{array}$ \\
\hline 80. Staff's empathy towards patients. & $\begin{array}{l}\square 1 \\
\square 5\end{array}$ & $\begin{array}{l}\square 2 \\
\square 6\end{array}$ & $\begin{array}{l}\square 3 \square 4 \\
\square 7\end{array}$ \\
\hline 81. Staff's skills in performing their roles. & $\begin{array}{l}\square 1 \\
\square 5\end{array}$ & $\begin{array}{l}\square 2 \\
\square 6\end{array}$ & $\begin{array}{l}\square 3 \square 4 \\
\square 7\end{array}$ \\
\hline 82. Staff's cultural competence. & $\begin{array}{l}\square 1 \\
\square 5\end{array}$ & $\begin{array}{l}\square 2 \\
\square 6\end{array}$ & $\begin{array}{l}\square 3 \square 4 \\
\square 7\end{array}$ \\
\hline $\begin{array}{l}\text { 83. Staff's knowledge about the organization's } \\
\text { processes and programs. }\end{array}$ & $\begin{array}{l}\square 1 \\
\square 5\end{array}$ & $\begin{array}{l}\square 2 \\
\square 6\end{array}$ & $\begin{array}{l}\square 3 \square 4 \\
\square 7\end{array}$ \\
\hline
\end{tabular}


Appendix D: Document Review Tool 
Organizational factors in HRSA-funded CHCS

DOCUMENTATION REVIEW/CONTENT ANALYSIS

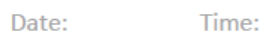

Health Center ID:

Organizational factors and dynamics that drive successful PCMH implementation and improved chronic disease management DOCUMENTATION REVIEW/CONTENT ANALYSIS DATA COLLECTION TOOL

INSTRUCTIONS: Use this tool to record observations, topics, and categories during the review of PMCH-related documents at the health center visited. The main focus of observation and review is the implementation of the PCMH model and related chronic disease management support efforts.

\begin{tabular}{|c|c|}
\hline $\begin{array}{l}\text { Patient flow } \\
\text { How patients move from one area to the other-e.g., from } \\
\text { registration to vitals, from vitals to different encounters }\end{array}$ & Elements observed in the text: \\
\hline $\begin{array}{l}\text { Announcements/communications (written, visual, or } \\
\text { electronic) available on site } \\
\text { a) Contents, topics, or issues } \\
\text { b) Are all available in English and any other languages (e.g., } \\
\text { Spanish, Creole, Portuguese, among others)? } \\
\text { c) Level of readability } \\
\text { d) Content appropriateness } \\
\text { e) Cultural sensitivity } \\
\text { f) Announcements/communications to educate patients on } \\
\text { health-related issues }\end{array}$ & Elements observed in the text: \\
\hline $\begin{array}{l}\text { Use of technology (e.g., practice management systems, } \\
\text { electronic health record, electronic prescribing, others) }\end{array}$ & Elements observed in the text: \\
\hline
\end{tabular}


Organizational factors in HRSA-funded CHCs

INTERVENTION CHARACTERISTICS

(Observations on the implementation of the PCMH model and chronic disease management/support efforts)

\section{COMPREHENSIVE CARE}

a) Comprehensiveness of services provided (physical,

mental/behavioral health care needs, including prevention and

wellness; acute care; and chronic care)

b) Team-based care (multidisciplinary team-based care, health care team composition, roles and responsibilities)

c) Comprehensive care strategies documented

d) Comprehensive health assessment

e) Care coordination (internal and external)

PATIENT-CENTERED CARE AND POPULATION MANAGEMENT

Elements observed in the text:

a) Relationship-based care; partnerships with patients and

their families; understanding and respect for patient's needs,

culture, values, and preferences.

b) Management of patient populations (e.g., comprehensive assessment and management of the health needs of defined

groups of patients, such as patients with hypertension or

diabetes)

c) Identification of patients who would benefit from care

management; planning, management and coordination of care

based on needs and patient characteristics (behavioral

considerations, complexity, social determinants of health, 
Organizational factors in HRSA-funded CHCS DOCUMENTATION REVIEW/CONTENT ANALYSIS

Date: Time:

\section{CARE PLANNING, MANAGEMENT, AND SUPPORT}

Health Center ID:

a) Collaboration between care team and patient/ family

/caregiver to develop and update care plan, incorporating

patient preferences, functional and lifestyle goals, treatment

goals, potential barriers, and self-care plan

b) Self-management support plan

c) Shared decision-making

d) Link with community resources

\section{Elements observed in the text:}

ENHANCED ACCESS (Ways CHC enhances access to care)

Elements observed in the text:

a) Walk-in/same-day patients accepted

b) Providing routine and urgent-care appointments outside

regular business hours (including weekends)

c) Alternative clinical encounters (e.g., real-time

communication, email, group visits, shared appointments)

d) 24/7 Access to Clinical Advice (e.g., clinical advice by

telephone or using a secure, interactive electronic system)

e) Patients have online access to their health information and

can request appointments, prescription refills, referrals and

test results.

f) Patients have two-way communication with the practice. 
Organizational factors in HRSA-funded CHCs

DOCUMENTATION REVIEW/CONTENT ANALYSIS

Date: Time:

\section{TEAM-BASED CARE}

a) Use of a culturally and linguistically appropriate, team-

based approach

b) Defined health care team for each personal clinician ( the

team includes a primary clinician and associated providers,

including behavioral health providers, and support staff who

work with the clinician)

c) Scheduled patient care team meetings or a structured

communication process focused on individual patient care

d) Members of the care team trained and assigned to

coordinate care for individual patients

e) Members of the care team assigned and trained to support

patients/families/caregivers in self-management, self-efficacy

and behavior change

\section{MEDICAL HOME RESPONSIBILITIES}

a) Coordination of patient care across multiple settings

b) Access to evidence-based care, patient/family education and self-management support

c) Comprehensiveness of the scope of services

d) Equal access to all patients regardless of source of payment 
Organizational factors in HRSA-funded CHCs

Date: Time:

DOCUMENTATION REVIEW/CONTENT ANALYSIS

Health Center ID:

\section{PATIENT SUPPORT SERVICES}

Elements observed in the text:

\section{Transportation services}

Health care coverage assistance (e.g., health care coverage

assistance or support staff available; announcements

indicating availability of this service; any patient being offered

the service; any patient indicating that he/she has received the

service)

Translation/interpretation services (Other languages, sign language)

Other services to remove barriers to care (e.g., financial

assistance/counseling, sliding fee discounts, case management,

care coordination, others)

CULTURALLY AND LINGUISTICALLY APPROPRIATE SERVICES

Elements observed in the text:

a) Assessment of population diversity and language needs

b) Emphasis on communication strategies and services that match the patient's language needs and preferences

\section{STRATEGIC PARTNERSHIPS}

Elements observed in the text:

(e.g., collaborations at the community level or the health care

system level - other providers) 
Organizational factors in HRSA-funded $\mathrm{CHCS}$

DOCUMENTATION REVIEW/CONTENT ANALYSIS

Health Center ID:

\section{PLANNING \\ Existence of an implementation plan or strategic plan that}

Elements observed in the text:

incorporates the implementation of the PCMH or chronic

disease management/support plan

\section{GUIDANCE AND LEADERSHIP}

Elements observed in the text:

Appointment of champions to facilitate model/initiative

implementation, champion behaviors/roles

Patient participation in the implementation of the model or

chronic disease management/support efforts

\section{Elements observed in the text:}

\section{COMMUNICATION}

Elements observed in the text:

Communication strategies or events related to the model and

chronic disease management/support efforts; communications

about implementation results 
Organizational factors in HRSA-funded CHCS

\section{INNER ORGANIZATIONAL SETTING}

\section{STRUCTURAL CHARACTERISTICS}

Elements observed in the text:

a) How tasks and responsibilities are divided to accomplish

objectives

b) Roles for clinical and nonclinical team members

c) Team structure

d) Staff leading and sustaining team based care

\section{NETWORKS \& COMMUNICATIONS}

Elements observed in the text:

a) Working relationships among health center staff and with leaders

b) Use of meetings; structure, frequency and focus of meetings

c) Meetings/relationships with key stakeholders (e.g.

community providers and leaders)

\section{CULTURE}

a) Observations about the culture of the organization (team

culture, hierarchical, flexible, entrepreneurial, rational,

internal/external focus);

b) Organizational beliefs, norms, values, assumptions,

embracing of new ideas/ change, level of control,

receptivity 
Organizational factors in HRSA-funded CHCs

DOCUMENTATION REVIEW/CONTENT A

Health Center ID:

\author{
ORGANIZATIONAL DYNAMICS \\ a) Work processes and practices (work flow) \\ b) Incentives and rewards for staff \\ c) Collaboration \\ d) Staff involvement \\ e) Shared planning and decision-making
}

Elements observed in the text:

\title{
LEARNING CLIMATE
}

Elements observed in the text:

a) Quality improvement initiatives, process/performance

improvement activities/efforts, staff training

b) Use of performance data to identify opportunities for

improvement and acts to improve clinical quality, efficiency and patient experience

c) Involvement of care team staff in the health center's performance evaluation and quality improvement activities

d) Involvement of patients/families/caregivers in quality improvement activities

\section{LEADERSHIP ENGAGEMENT}

Elements observed in the text:

a) Level of involvement of leaders

b) Barriers and facilitators created by leaders 
Organizational factors in HRSA-funded CHCs

DOCUMENTATION REVIEW/CONTENT ANALYSIS

RESOURCES

a) Health center's resources to implement the model and

carry out chronic disease management and support

activities (human, time, infrastructure, equipment, funding

resources)

Elements observed in the text:

Health Center ID

\begin{tabular}{l|l|l|} 
& & \\
CHARACTERISTICS OF INDIVIDUALS & Elements observed in the text: \\
$\begin{array}{l}\text { a) Approach to care } \\
\text { b) Team cohesiveness } \\
\text { c) Staff roles } \\
\begin{array}{l}\text { d) Skills (job-related, interpersonal, cultural, language, } \\
\text { among others) }\end{array}\end{array}$ \\
\hline OTHER
\end{tabular}


Appendix E: Interview Questions

\begin{tabular}{|c|c|}
\hline $\begin{array}{l}\text { Organizational } \\
\text { culture }\end{array}$ & $\begin{array}{l}\text { 1. Tell me about your organization. } \\
\text { 2. How would you describe it? } \\
\text { 3. How would you define the people working at the } \\
\text { organization? }\end{array}$ \\
\hline $\begin{array}{l}\text { Patient Centered } \\
\text { Medical Home } \\
\text { approach }\end{array}$ & $\begin{array}{l}\text { 4. How would you define "patient-centered"? } \\
\text { 5. What makes this a patient-centered organization? } \\
\text { 6. What do you do to maintain the "patient-centeredness" of } \\
\text { the health center? } \\
\text { 7. Does this focus you're describing fit the characteristics or } \\
\text { essence of your organization? How? }\end{array}$ \\
\hline $\begin{array}{l}\text { PCMH and Chronic } \\
\text { Disease Management }\end{array}$ & $\begin{array}{l}\text { 8. How does this "patient-centered" approach you are } \\
\text { describing relate to your work with chronic disease } \\
\text { management? } \\
\text { 9. How do you use that "patient-centered" strategy to support } \\
\text { chronic disease management? (Examples) } \\
\text { 10. Describe what your organization does to support chronic } \\
\text { disease management } \\
\text { 11. What is and how do you see your role in this process? }\end{array}$ \\
\hline $\begin{array}{l}\mathrm{PMCH} \\
\text { Implementation }\end{array}$ & $\begin{array}{l}\text { 12. Tell me about your experience implementing the Patient- } \\
\text { Centered Medical Home model } \\
\text { 13. What were some of the key changes you had to go } \\
\text { through? How did you manage this change? } \\
\text { 14. The model has several components and as part of the } \\
\text { implementation. Can you tell me about how your } \\
\text { organization covers these components? }\end{array}$ \\
\hline $\begin{array}{l}\text { Barriers and } \\
\text { facilitators }\end{array}$ & $\begin{array}{l}\text { 15. Let's talk about the things or factors you believe } \\
\text { contributed to the implementation of the PCMH model } \\
\text { (anything in particular that you think facilitated the } \\
\text { process). Which organizational attributes do you think } \\
\text { contributed to this process? } \\
\text { 16. Now, let's think about barriers or obstacles encountered } \\
\text { along the way. Which factors or issues do you believe } \\
\text { make it difficult to implement the components of this } \\
\text { model? } \\
\text { 17. Tell me about your roles in this process. } \\
\text { 18. What can you say about the role of the health center's } \\
\text { leadership in this process? }\end{array}$ \\
\hline
\end{tabular}


Appendix G: Case 1 Emerging Themes and Key Terms by Source of Data

\begin{tabular}{|c|c|c|c|}
\hline $\begin{array}{c}\text { Emerging } \\
\text { themes }\end{array}$ & Observations & Interviews & $\begin{array}{c}\text { Document } \\
\text { Review/Analysis }\end{array}$ \\
\hline $\begin{array}{l}\text { 1. Patient- } \\
\text { centered, need- } \\
\text { based care }\end{array}$ & $\begin{array}{l}\text { Patient/patients } \\
\text { Patient at the center } \\
\text { Patient-centered } \\
\text { care } \\
\text { Patient needs } \\
\text { Patient-centered } \\
\text { care }\end{array}$ & $\begin{array}{l}\text { Patient }(\mathrm{s}) \\
\text { Patient needs } \\
\text { Patient-centered care }\end{array}$ & $\begin{array}{l}\text { Patient(s) } \\
\text { Patient-centered } \\
\text { Patient needs } \\
\text { Patient history } \\
\text { Assessment of } \\
\text { behaviors affecting } \\
\text { health } \\
\text { Social Assessment }\end{array}$ \\
\hline $\begin{array}{l}\text { 2. Medical } \\
\text { Home } \\
\text { approach and } \\
\text { implementation }\end{array}$ & $\begin{array}{l}\text { Medical home } \\
\text { Model } \\
\text { Activities/events } \\
\text { Implementation }\end{array}$ & $\begin{array}{l}\text { Medical home/PCMH } \\
\text { Implementation/Imple } \\
\text { ment } \\
\text { Barriers/ Facilitators } \\
\text { PCMH Program } \\
\text { Money/Funding/Resour } \\
\text { ces }\end{array}$ & $\begin{array}{l}\text { Medical } \\
\text { home/PCMH } \\
\text { PCMH standards, } \\
\text { elements, factors } \\
\text { Guidelines, tools } \\
\text { Policies }\end{array}$ \\
\hline $\begin{array}{l}\text { 3. Care } \\
\text { Coordination }\end{array}$ & $\begin{array}{l}\text { Care coordination } \\
\text { Referrals } \\
\text { Pre-planned visits }\end{array}$ & $\begin{array}{l}\text { Referrals } \\
\text { Authorizations } \\
\text { Coordination } \\
\text { Coordinators } \\
\end{array}$ & $\begin{array}{l}\text { Care coordination } \\
\text { Patient support } \\
\text { Support process of } \\
\text { care }\end{array}$ \\
\hline $\begin{array}{l}\text { 4. Health } \\
\text { center as } \\
\text { organizational } \\
\text { system }\end{array}$ & $\begin{array}{l}\text { Organization } \\
\text { System } \\
\text { Leadership } \\
\text { Management } \\
\text { Managers } \\
\text { Departments }\end{array}$ & $\begin{array}{l}\text { Department(s) } \\
\text { Organizational } \\
\text { Areas/functions } \\
\text { Organizational } \\
\text { Structure } \\
\text { Manager } \\
\text { Management/Leadershi } \\
\text { p } \\
\text { CEO, COO }\end{array}$ & $\begin{array}{l}\text { Organizational } \\
\text { structure } \\
\text { Departments } \\
\text { Leadership } \\
\text { Management }\end{array}$ \\
\hline $\begin{array}{l}5 . \\
\text { Organizational } \\
\text { and Leadership } \\
\text { Culture }\end{array}$ & $\begin{array}{l}\text { Organizational } \\
\text { culture } \\
\text { Leadership } \\
\text { behaviors } \\
\text { Staff interactions } \\
\text { Staff characteristics }\end{array}$ & $\begin{array}{l}\text { Organizational culture } \\
\text { Leadership behaviors }\end{array}$ & $\begin{array}{l}\text { Leadership culture } \\
\text { Team-based culture } \\
\text { Organizational } \\
\text { culture } \\
\text { Learning culture }\end{array}$ \\
\hline $\begin{array}{l}\text { 7. Team-based } \\
\text { care }\end{array}$ & $\begin{array}{l}\text { Team } \\
\text { Doctor } \\
\text { Physician } \\
\text { Provider } \\
\text { Team members } \\
\text { Coordinators } \\
\text { (medical home, } \\
\end{array}$ & $\begin{array}{l}\text { Team } \\
\text { Doctor, physician } \\
\text { Provider(s) } \\
\text { Staff } \\
\text { Support } \\
\text { Nurse }\end{array}$ & $\begin{array}{l}\text { Team } \\
\text { Primary Care } \\
\text { Provider } \\
\text { Manager } \\
\text { Coordinators } \\
\text { Team-based care }\end{array}$ \\
\hline
\end{tabular}




\begin{tabular}{|c|c|c|c|}
\hline & $\begin{array}{l}\text { referral, care } \\
\text { coordinators) } \\
\text { Nurse/ head nurse } \\
\text { Front desk staff } \\
\text { Manager } \\
\text { Assistant } \\
\text { Meetings, Huddles }\end{array}$ & $\begin{array}{l}\text { Medical home } \\
\text { coordinators } \\
\text { Referral coordinators } \\
\text { Coordinators } \\
\text { Medical Home } \\
\text { Coordinators } \\
\text { Referral coordinators } \\
\text { Meetings }\end{array}$ & $\begin{array}{l}\text { Clinical support } \\
\text { staff } \\
\text { Patient support } \\
\text { staff } \\
\text { Behavioral health } \\
\text { providers } \\
\text { Meetings (huddles, } \\
\text { medical home } \\
\text { team) } \\
\text { Pharmacy } \\
\text { involvement in } \\
\text { health care team }\end{array}$ \\
\hline $\begin{array}{l}\text { 8. Access to } \\
\text { care }\end{array}$ & $\begin{array}{l}\text { Available } \\
\text { Availability } \\
\text { Access } \\
\text { Accessible } \\
\text { Appointment } \\
\text { Patient visits } \\
\text { Walk-in visits } \\
\text { Same day visits }\end{array}$ & $\begin{array}{l}\text { Appointment(s) } \\
\text { Visits } \\
\text { Schedule }\end{array}$ & $\begin{array}{l}\text { Appointments } \\
\text { Access } \\
\text { Availability } \\
\text { Enhanced access } \\
\text { Timely } \\
\text { Visits } \\
\text { Scheduling } \\
\text { Walk-in same day }\end{array}$ \\
\hline $\begin{array}{l}9 . \\
\text { Comprehensive } \\
\text { care }\end{array}$ & $\begin{array}{l}\text { Services } \\
\text { Service delivery } \\
\text { Assistance } \\
\text { Services available }\end{array}$ & $\begin{array}{l}\text { Service delivery } \\
\text { Services provided }\end{array}$ & $\begin{array}{l}\text { Service delivery } \\
\text { Services } \\
\text { Referrals } \\
\text { Patient support } \\
\text { services } \\
\text { Screenings } \\
\text { Case management } \\
\text { Behavioral health } \\
\text { services }\end{array}$ \\
\hline $\begin{array}{l}\text { 10. Patient } \\
\text { support }\end{array}$ & $\begin{array}{l}\text { Patient support } \\
\text { services } \\
\text { Financial support } \\
\text { Sliding fee } \\
\text { discounts } \\
\text { Interpretation } \\
\text { services } \\
\text { Accessibility } \\
\text { Support staff }\end{array}$ & $\begin{array}{l}\text { Patient support } \\
\text { Transportation } \\
\text { Translation } \\
\text { Financial assistance } \\
\text { Support with food } \\
\text { program } \\
\text { Support with health } \\
\text { coverage } \\
\text { Patient support staff }\end{array}$ & $\begin{array}{l}\text { Enabling, patient } \\
\text { support services } \\
\text { Translation } \\
\text { Sliding fee } \\
\text { discount } \\
\text { Health coverage } \\
\text { assistance }\end{array}$ \\
\hline $\begin{array}{l}\text { 11. Culturally } \\
\text { and } \\
\text { linguistically } \\
\text { appropriate } \\
\text { services }\end{array}$ & $\begin{array}{l}\text { Languages } \\
\text { Spanish/English/Cre } \\
\text { ole } \\
\text { Interpretation } \\
\text { services }\end{array}$ & Interpretation services & $\begin{array}{l}\text { Languages } \\
\text { (English, Spanish, } \\
\text { Creole) } \\
\text { Interpretation } \\
\text { services }\end{array}$ \\
\hline
\end{tabular}




\begin{tabular}{|c|c|c|c|}
\hline & & & $\begin{array}{l}\text { Communication } \\
\text { needs and } \\
\text { preferences } \\
\text { Cultural diversity } \\
\text { (patients/ staff) } \\
\text { Cultural sensitivity } \\
\text { Cultural } \\
\text { characteristics } \\
\text { Cultural } \\
\text { competence }\end{array}$ \\
\hline $\begin{array}{l}12 . \\
\text { Patient/family } \\
\text { participation in } \\
\text { the care } \\
\text { process }\end{array}$ & $\begin{array}{l}\text { Patients } \\
\text { asking/calling } \\
\text { Patient involvement } \\
\text { Patients taking care } \\
\text { of themselves } \\
\text { Patients involved in } \\
\text { their care } \\
\text { Patient-staff } \\
\text { interaction } \\
\text { Patient roles and } \\
\text { responsibilities }\end{array}$ & $\begin{array}{l}\text { Family participation } \\
\text { Patient meetings with } \\
\text { the health care team }\end{array}$ & $\begin{array}{l}\text { Include, involve } \\
\text { Inclusive process } \\
\text { Patient/family } \\
\text { involvement } \\
\text { Participation } \\
\text { Caregivers } \\
\text { Patient roles and } \\
\text { responsibilities }\end{array}$ \\
\hline $\begin{array}{l}\text { 13. Patient } \\
\text { Communicatio } \\
\mathrm{n} \text { and } \\
\text { Education }\end{array}$ & $\begin{array}{l}\text { Announcements } \\
\text { Communicate, } \\
\text { communication } \\
\text { Communication } \\
\text { with patients } \\
\text { Messages } \\
\text { Screens, } \\
\text { Displays, signs } \\
\text { Posters } \\
\text { Information } \\
\text { Instruct, instruction } \\
\text { Education } \\
\text { Educational, } \\
\text { Educate }\end{array}$ & $\begin{array}{l}\text { Communications } \\
\text { Communication with } \\
\text { the patient } \\
\text { Phones } \\
\text { Information } \\
\text { Education }\end{array}$ & $\begin{array}{l}\text { Information } \\
\text { Informed patient } \\
\text { Provider/staff- } \\
\text { patient } \\
\text { communication) } \\
\text { Communications } \\
\text { Assessing } \\
\text { information/ } \\
\text { education needs } \\
\text { Educational, } \\
\text { Educate, } \\
\text { Resources and } \\
\text { information } \\
\text { Health literacy } \\
\text { assessment } \\
\text { Assessing } \\
\text { understanding of } \\
\text { medication } \\
\text { Community events } \\
\text { Community } \\
\text { participation }\end{array}$ \\
\hline $\begin{array}{l}\text { 14. Use of } \\
\text { technology to }\end{array}$ & $\begin{array}{l}\text { Technology } \\
\text { System }\end{array}$ & $\begin{array}{l}\text { Technology Systems } \\
\text { Practice Management } \\
\text { system }\end{array}$ & $\begin{array}{l}\text { Technology } \\
\text { systems }\end{array}$ \\
\hline
\end{tabular}




\begin{tabular}{|c|c|c|c|}
\hline $\begin{array}{l}\text { facilitate } \\
\text { patient care }\end{array}$ & $\begin{array}{l}\text { Information } \\
\text { management }\end{array}$ & $\begin{array}{l}\text { Electronic health record } \\
\text { Facilitator }\end{array}$ & $\begin{array}{l}\text { Support decision } \\
\text { making at the point } \\
\text { of care } \\
\text { Self-care support } \\
\text { Electronic health } \\
\text { record } \\
\text { Use of } \\
\text { technologies }\end{array}$ \\
\hline $\begin{array}{l}\text { 15. Population } \\
\text { health } \\
\text { management }\end{array}$ & & $\begin{array}{l}\text { Population health } \\
\text { management }\end{array}$ & $\begin{array}{l}\text { Population } \\
\text { Population health } \\
\text { management } \\
\text { Identifying patient } \\
\text { populations } \\
\text { (panels) } \\
\text { Identifying } \\
\text { population needs } \\
\text { Population health } \\
\text { initiatives } \\
\text { Vulnerable } \\
\text { populations } \\
\text { Transient } \\
\text { populations }\end{array}$ \\
\hline $\begin{array}{l}16 . \\
\text { Performance } \\
\text { and Quality } \\
\text { Improvement }\end{array}$ & $\begin{array}{l}\text { Improvement } \\
\text { Health improvement } \\
\text { Quality } \\
\text { improvement } \\
\text { Performance } \\
\text { improvement } \\
\text { Success } \\
\text { Goals }\end{array}$ & $\begin{array}{l}\text { Quality } \\
\text { Quality improvement } \\
\text { Performance } \\
\text { improvement } \\
\text { Improving, } \\
\text { improvements } \\
\text { Monitor, monitoring, } \\
\text { Reports }\end{array}$ & $\begin{array}{l}\text { Quality } \\
\text { improvement } \\
\text { Reporting } \\
\text { Review } \\
\text { Improvement, } \\
\text { improving } \\
\text { Performance } \\
\text { Monitoring } \\
\text { Self-assessment } \\
\text { Compliance } \\
\text { Use of tools } \\
\text { Training } \\
\text { Plan Do Study Act } \\
\text { (PDSA) }\end{array}$ \\
\hline $\begin{array}{l}\text { 17. Planned } \\
\text { Care/Care } \\
\text { Planning }\end{array}$ & $\begin{array}{l}\text { Pre-planned visits } \\
\text { Team huddles }\end{array}$ & Pre-planned visits & $\begin{array}{l}\text { Plan(s)/ Planning } \\
\text { Health care plan } \\
\text { Treatment plan } \\
\text { Planned care } \\
\text { Self-management } \\
\text { plan } \\
\text { Strategies planned } \\
\text { Follow-up plan }\end{array}$ \\
\hline
\end{tabular}




\begin{tabular}{|c|c|c|c|}
\hline & & & $\begin{array}{l}\text { Health education } \\
\text { plan } \\
\text { Pre-planned visits }\end{array}$ \\
\hline $\begin{array}{l}\text { 18. Chronic } \\
\text { disease } \\
\text { management } \\
\text { and self- } \\
\text { management/se } \\
\text { lf-care support }\end{array}$ & $\begin{array}{l}\text { Diabetes } \\
\text { Chronic } \\
\text { disease/condition } \\
\text { Diabetic patients } \\
\text { Diabetes } \\
\text { management } \\
\text { Diabetes care } \\
\text { Self-management }\end{array}$ & $\begin{array}{l}\text { Diabetes } \\
\text { Diabetic } \\
\text { Individual and group } \\
\text { education for diabetes } \\
\text { and hypertension }\end{array}$ & $\begin{array}{l}\text { Chronic conditions } \\
\text { Chronic disease } \\
\text { management } \\
\text { Preventing } \\
\text { complications } \\
\text { Diabetes, } \\
\text { hypertension, } \\
\text { asthma control) } \\
\text { Self-care } \\
\text { Self-management } \\
\text { Self-management } \\
\text { goals } \\
\text { Self-care support } \\
\text { Patient } \\
\text { compliance/adhere } \\
\text { nce } \\
\text { Self-care planning } \\
\text { Patient goals } \\
\text { Provider goals for } \\
\text { patient health }\end{array}$ \\
\hline $\begin{array}{l}\text { 19. Community } \\
\text { partnerships }\end{array}$ & $\begin{array}{l}\text { Community } \\
\text { organizations } \\
\text { Partnerships }\end{array}$ & $\begin{array}{l}\text { Partnerships with } \\
\text { community } \\
\text { organizations }\end{array}$ & $\begin{array}{l}\text { Links with } \\
\text { community } \\
\text { resources } \\
\text { Strategic } \\
\text { community } \\
\text { partnerships }\end{array}$ \\
\hline
\end{tabular}


Appendix H: Case 2 Emerging Themes and Key Terms by Source of Data

\begin{tabular}{|c|c|c|c|}
\hline Emerging themes & Observations & $\begin{array}{c}\text { Document } \\
\text { Review/Analysis }\end{array}$ & Interviews \\
\hline $\begin{array}{l}\text { 1. Patient-centered, } \\
\text { need-based care }\end{array}$ & $\begin{array}{l}\text { Patient Service area } \\
\text { Vulnerable } \\
\text { populations } \\
\text { Patient experience }\end{array}$ & $\begin{array}{l}\text { Patient, patients } \\
\text { Patient needs } \\
\text { Patient flow, } \\
\text { experience } \\
\text { Population health } \\
\text { needs } \\
\text { Vulnerable } \\
\text { populations }\end{array}$ & $\begin{array}{l}\text { Patient-centered } \\
\text { care } \\
\text { Vulnerable } \\
\text { populations } \\
\text { CHC model } \\
\text { Patient } \\
\text { experience, } \\
\text { satisfaction }\end{array}$ \\
\hline $\begin{array}{l}\text { 2. Medical Home } \\
\text { approach and } \\
\text { implementation }\end{array}$ & $\begin{array}{l}\text { Medical home } \\
\text { model }\end{array}$ & $\begin{array}{l}\text { Site level } \\
\text { implementation } \\
\text { Policies established } \\
\text { PCMH self- } \\
\text { assessment } \\
\text { Planning } \\
\text { PCMH leadership } \\
\text { Resources invested } \\
\text { Challenges/barriers } \\
\text { Accomplishments } \\
\text { Facilitators }\end{array}$ & $\begin{array}{l}\text { Barriers } \\
\text { Facilitators } \\
\text { PCMH structure } \\
\text { Patient Flow } \\
\text { PCMH model } \\
\text { PCMH } \\
\text { recognition } \\
\text { Standards, } \\
\text { guidelines, } \\
\text { requirements } \\
\text { PCMH team } \\
\text { Planning } \\
\text { Site } \\
\text { implementation } \\
\text { Organizational } \\
\text { implementation } \\
\text { Flexible } \\
\text { implementation } \\
\text { Quality } \\
\text { recognition } \\
\text { External experts } \\
\text { Resources } \\
\text { Funding and } \\
\text { equipment }\end{array}$ \\
\hline 3. Care Coordination & $\begin{array}{l}\text { Patient Service } \\
\text { officers } \\
\text { Referrals } \\
\text { Patient Flow }\end{array}$ & $\begin{array}{l}\text { Coordination } \\
\text { Referrals } \\
\text { Patient Service } \\
\text { Officers } \\
\text { Care transitions } \\
\text { Tracking and follow- } \\
\text { up } \\
\text { Community resources } \\
\text { Hospital }\end{array}$ & $\begin{array}{l}\text { Patient care } \\
\text { cycle } \\
\text { Care } \\
\text { coordination } \\
\text { Internal referral } \\
\text { External referral } \\
\text { Referral process } \\
\text { Patient Service } \\
\text { officers }\end{array}$ \\
\hline
\end{tabular}




\begin{tabular}{|c|c|c|c|}
\hline & & & $\begin{array}{l}\text { Patient flow } \\
\text { Tracking } \\
\text { Follow-up } \\
\text { Documentation }\end{array}$ \\
\hline $\begin{array}{l}\text { 4. Organizational } \\
\text { system }\end{array}$ & $\begin{array}{l}\text { Site, Facilities } \\
\text { Waiting rooms } \\
\text { Functional areas } \\
\text { Departments } \\
\text { Outpatient } \\
\text { Department } \\
\text { Registration } \\
\text { Administration }\end{array}$ & $\begin{array}{l}\text { Health center } \\
\text { organization } \\
\text { Sites } \\
\text { Organizational } \\
\text { structure }\end{array}$ & $\begin{array}{l}\text { Sites } \\
\text { Staff resources } \\
\text { Administration } \\
\text { Physical } \\
\text { infrastructure }\end{array}$ \\
\hline $\begin{array}{l}\text { 5. Organizational } \\
\text { and Leadership } \\
\text { Culture }\end{array}$ & $\begin{array}{l}\text { Administration } \\
\text { Health Center } \\
\text { Director } \\
\text { Nursing Supervisor } \\
\text { Management, } \\
\text { manager } \\
\text { Staff behaviors } \\
\text { Decision-making } \\
\text { Leadership } \\
\text { behaviors }\end{array}$ & $\begin{array}{l}\text { Organizational } \\
\text { culture } \\
\text { Leadership } \\
\text { Management, } \\
\text { managers } \\
\text { PCMH Coordinator } \\
\text { Team leaders } \\
\text { Champions } \\
\text { Privacy } \\
\text { Officer/Health } \\
\text { Information Manager }\end{array}$ & $\begin{array}{l}\text { Organizational } \\
\text { culture } \\
\text { Culture } \\
\text { transformation } \\
\text { Leadership } \\
\text { culture } \\
\text { Administration } \\
\text { Medical Director } \\
\text { Nursing } \\
\text { leadership } \\
\text { Health } \\
\text { Information } \\
\text { Manager } \\
\text { Corporate } \\
\text { Committee } \\
\text { Corporate } \\
\text { Leadership } \\
\text { Executive } \\
\text { Director } \\
\text { Board members }\end{array}$ \\
\hline 7. Team-based care & $\begin{array}{l}\text { Health educator } \\
\text { Nurse } \\
\text { Social Worker } \\
\text { Nutritionist } \\
\text { Pediatricians } \\
\text { Primary care } \\
\text { doctors }\end{array}$ & $\begin{array}{l}\text { Provider } \\
\text { Multidisciplinary } \\
\text { team } \\
\text { Nurses } \\
\text { Health educators } \\
\text { Social worker } \\
\text { Nutritionist } \\
\text { Specialists } \\
\text { Team meetings }\end{array}$ & $\begin{array}{l}\text { Doctor } \\
\text { Nutritionist } \\
\text { Health Educator } \\
\text { Nurse } \\
\text { Medical Services } \\
\text { Director } \\
\text { Team } \\
\text { communication } \\
\text { Teamwork } \\
\text { Collaboration } \\
\text { Clinical team } \\
\text { meetings }\end{array}$ \\
\hline
\end{tabular}




\begin{tabular}{|c|c|c|c|}
\hline & & & $\begin{array}{l}\text { Interprofessional } \\
\text { communication } \\
\text { Multidisciplinary } \\
\text { team } \\
\text { Patient Service } \\
\text { officer } \\
\text { Social Worker } \\
\text { Allied health } \\
\text { professions } \\
\text { Team huddles } \\
\end{array}$ \\
\hline 8. Access to care & $\begin{array}{l}\text { Hours of service } \\
\text { Wait time } \\
\text { Appointment } \\
\text { scheduling }\end{array}$ & $\begin{array}{l}\text { Service hours } \\
\text { Extended hours } \\
\text { Appointments } \\
\text { Schedule } \\
\text { Walk-in/same-day } \\
\text { After hours care } \\
\text { Phone triage }\end{array}$ & $\begin{array}{l}\text { Appointments } \\
\text { No show } \\
\text { Availability } \\
\text { Outpatient } \\
\text { Department } \\
\text { Phone triage } \\
\text { Wait time }\end{array}$ \\
\hline $\begin{array}{l}\text { 9. Comprehensive } \\
\text { care }\end{array}$ & $\begin{array}{l}\text { Health services } \\
\text { Prenatal care } \\
\text { Pediatrics } \\
\text { Immunization } \\
\text { Specialized care } \\
\text { Pharmacy } \\
\text { Community events }\end{array}$ & $\begin{array}{l}\text { Comprehensiveness } \\
\text { Health services } \\
\text { Prevention } \\
\text { Screenings }\end{array}$ & $\begin{array}{l}\text { Medical services } \\
\text { Health education } \\
\text { Nutrition } \\
\text { Community } \\
\text { health clinics } \\
\text { Screenings, } \\
\text { immunization } \\
\text { Mobile unit } \\
\text { Pharmacy }\end{array}$ \\
\hline 10. Patient support & $\begin{array}{l}\text { Patient support } \\
\text { services } \\
\text { Interpretation } \\
\text { services Sliding Fee } \\
\text { Discount }\end{array}$ & $\begin{array}{l}\text { Transportation } \\
\text { Interpretation } \\
\text { Support, enabling } \\
\text { services } \\
\text { Outreach and } \\
\text { Enrollment }\end{array}$ & $\begin{array}{l}\text { Enabling, } \\
\text { support services } \\
\text { Patient transport } \\
\text { Health coverage } \\
\text { assistance } \\
\text { Home visits }\end{array}$ \\
\hline $\begin{array}{l}\text { 11. Culturally and } \\
\text { linguistically } \\
\text { appropriate services }\end{array}$ & $\begin{array}{l}\text { Language } \\
\text { Spanish/English } \\
\text { Interpretation } \\
\text { services } \\
\text { Patient culture } \\
\text { Cultural behaviors } \\
\text { Culturally- } \\
\text { appropriate events } \\
\text { Cultural } \\
\text { homogeneity }\end{array}$ & $\begin{array}{l}\text { Interpretation } \\
\text { services } \\
\text { Services for English } \\
\text { speakers } \\
\text { Culturally- } \\
\text { appropriate contents }\end{array}$ & $\begin{array}{l}\text { Communication } \\
\text { needs } \\
\text { Cultural } \\
\text { behaviors }\end{array}$ \\
\hline $\begin{array}{l}\text { 12. Patient/family } \\
\text { participation }\end{array}$ & $\begin{array}{l}\text { Patient events } \\
\text { Patient rights and } \\
\text { responsibilities }\end{array}$ & $\begin{array}{l}\text { Patient participation } \\
\text { Patient goals }\end{array}$ & $\begin{array}{l}\text { Family } \\
\text { participation }\end{array}$ \\
\hline
\end{tabular}




\begin{tabular}{|c|c|c|c|}
\hline & & $\begin{array}{l}\text { Patient/community } \\
\text { events }\end{array}$ & $\begin{array}{l}\text { Patient } \\
\text { participation } \\
\text { Patient surveys }\end{array}$ \\
\hline $\begin{array}{l}\text { 13. Communication } \\
\text { and Education }\end{array}$ & $\begin{array}{l}\text { Announcements } \\
\text { Communications } \\
\text { Information } \\
\text { Materials } \\
\text { Signs/posters } \\
\text { English/Spanish } \\
\text { Employee } \\
\text { communication } \\
\text { Educational events }\end{array}$ & $\begin{array}{l}\text { Health center } \\
\text { information } \\
\text { Patient information } \\
\text { Patient education } \\
\text { PCMH } \\
\text { communication } \\
\text { Languages }\end{array}$ & $\begin{array}{l}\text { Patient education } \\
\text { Educational talks } \\
\text { Patient events } \\
\text { Communication } \\
\text { Patient } \\
\text { understanding }\end{array}$ \\
\hline $\begin{array}{l}\text { 14. Use of } \\
\text { technology to } \\
\text { facilitate patient care }\end{array}$ & $\begin{array}{l}\text { Practice } \\
\text { management } \\
\text { system } \\
\text { Technology } \\
\text { systems }\end{array}$ & $\begin{array}{l}\text { Practice management } \\
\text { Electronic health } \\
\text { record } \\
\text { Patient assessment } \\
\text { Population health } \\
\text { management } \\
\text { Electronic } \\
\text { prescribing }\end{array}$ & $\begin{array}{l}\text { Electronic } \\
\text { system } \\
\text { Electronic } \\
\text { referral } \\
\text { Electronic health } \\
\text { record } \\
\text { Documentation } \\
\text { Electronic } \\
\text { prescribing } \\
\text { Information } \\
\text { Systems } \\
\text { Technology } \\
\text { infrastructure }\end{array}$ \\
\hline $\begin{array}{l}\text { 15. Population } \\
\text { health management }\end{array}$ & & $\begin{array}{l}\text { Managing patient } \\
\text { populations } \\
\text { Identifying } \\
\text { population needs } \\
\text { Vulnerable } \\
\text { populations } \\
\text { Population health } \\
\text { management } \\
\end{array}$ & $\begin{array}{l}\text { Identifying } \\
\text { population needs } \\
\text { Vulnerable } \\
\text { populations }\end{array}$ \\
\hline $\begin{array}{l}\text { 16. Learning culture, } \\
\text { performance } \\
\text { assessment, and } \\
\text { quality improvement }\end{array}$ & $\begin{array}{l}\text { Employee training } \\
\text { Staff meeting } \\
\text { Coaching }\end{array}$ & $\begin{array}{l}\text { Self-assessment } \\
\text { Quality improvement } \\
\text { Performance } \\
\text { improvement } \\
\text { Evidence-based } \\
\text { guidelines } \\
\text { Training } \\
\text { Evaluation }\end{array}$ & $\begin{array}{l}\text { Self-assessment } \\
\text { PCMH } \\
\text { compliance } \\
\text { Performance } \\
\text { improvement } \\
\text { Quality } \\
\text { Improvement } \\
\text { Performance } \\
\text { measures } \\
\text { Quality } \\
\text { Department }\end{array}$ \\
\hline
\end{tabular}




\begin{tabular}{|c|c|c|c|}
\hline & & & $\begin{array}{l}\text { Audits } \\
\text { Site visits } \\
\text { PDSA cycle } \\
\text { Training } \\
\text { Documentation }\end{array}$ \\
\hline $\begin{array}{l}\text { 17. Planned } \\
\text { Care/Care Planning }\end{array}$ & Care planning & $\begin{array}{l}\text { Care plan } \\
\text { Pre-planned visits } \\
\text { Pre-visit } \\
\text { questionnaire } \\
\text { Goals }\end{array}$ & $\begin{array}{l}\text { Care plan } \\
\text { Pre-planned } \\
\text { visits } \\
\text { Pre-clinics } \\
\text { Pre-visit } \\
\text { questionnaire }\end{array}$ \\
\hline $\begin{array}{l}\text { 18. Chronic disease } \\
\text { management and } \\
\text { self- } \\
\text { management/self- } \\
\text { care support }\end{array}$ & $\begin{array}{l}\text { Heart health } \\
\text { education } \\
\text { Mental health } \\
\text { education }\end{array}$ & $\begin{array}{l}\text { Diabetes } \\
\text { Hypertension } \\
\text { Obesity } \\
\text { Self-management } \\
\text { support } \\
\text { Medication } \\
\text { management } \\
\text { Medication } \\
\text { adherence } \\
\text { Patient goals }\end{array}$ & $\begin{array}{l}\text { Chronic } \\
\text { conditions } \\
\text { Patient } \\
\text { commitment } \\
\text { Patient } \\
\text { compliance } \\
\text { Behavioral } \\
\text { change } \\
\text { Patient education } \\
\text { Self-care support } \\
\text { Diabetes } \\
\text { Hypertension } \\
\text { Adherence to } \\
\text { treatment }\end{array}$ \\
\hline $\begin{array}{l}\text { 19. Strategic } \\
\text { partnerships }\end{array}$ & & $\begin{array}{l}\text { Community } \\
\text { partnerships }\end{array}$ & $\begin{array}{l}\text { Community } \\
\text { referrals } \\
\text { Community } \\
\text { events }\end{array}$ \\
\hline
\end{tabular}


VITA

WILLMARIE LATORRE

1998-2001

B.A., Communication

University of the Sacred Heart

San Juan, Puerto Rico

2002-2005

M.A., Communication

University of the Sacred Heart

San Juan, Puerto Rico

2004-2013

Strategic Organizational Consultant

Toa Alta, Puerto Rico

2006-2008

M.P.H., Health Promotion and Disease Prevention

Florida International University

Miami, Florida

2009

Certified in Public Health (CPH)

National Board of Public Health Examiners

2013-2019

Doctoral Candidate

Florida International University

Miami, Florida

2013-present

Public Health/Health Care Strategic Consultant

Miami, Florida

2014-2015

Grant Project Director

Ana G. Mendez University System

Miramar, Florida

2018

Senior Grant Developer

Public Health Trust/Jackson Health System

Miami, Florida

\section{PUBLICATIONS AND PRESENTATIONS}

Bastida, E., Kaur, R., Latorre, W., \& Alsaif, B. (2018, July). Becoming Agents of Family

Health Promotion: Mexican American Grandparents Increase Self-Efficacy, Educate

Grandchildren and Improve Physical Health. Presentation at the 2018 Healthy Aging

Summit, Washington, DC. 
Latorre, W. (2005). El rol de las relaciones públicas en las organizaciones sin fines de lucro dedicadas a la promoción de la salud (Master's Thesis). Universidad del Sagrado Corazón, San Juan, PR.

Latorre, W. (2010, May). Strategic Incorporation of the National Access to Care Goal to a Community Health Center's Plan: HRSA's 2015 Access to Care Plan. Conference conducted at the Puerto Rico Primary Care Association Assembly, Dorado, Puerto Rico.

Latorre, W. (2010, May). Effective Planning to Promote the Growth of a Community Health Center. Conference conducted at the Puerto Rico Primary Care Association Assembly, Dorado, Puerto Rico.

Latorre, W. (2011, September). Leadership as a Strategy to Improve Quality, Efficiency, and Effectiveness in a Community Health Center. Conference conducted at Puerto Rico Primary Care Association Clinical Leadership Seminar, Dorado, Puerto Rico.

Latorre, W. (2012, March). Effectively Leading the Transformation of the Health Care Model: Establishing a Patient-Centered Medical Home. Conference conducted at the meeting of Atlantic Medical Center, Barceloneta, Puerto Rico.

Latorre, W. (2012, September). Health Center Board Performance. Conference conducted at the Puerto Rico Primary Care Association Annual Convention, San Juan, Puerto Rico.

Latorre, W. (2012, September). Quality and Performance Improvement in a PatientCentered Medical Home. Conference conducted at the meeting of Corporacion de Servicios Medicos Primarios y Preventivos de Hatillo, Caguas, Puerto Rico.

Weissman, J., Preston, S., Sebekos, E., Latorre, W., Alsaif, B., Krupp, K., Darrow, W. (2016). Associations Between Health and Academic Success at a Florida University: An Exploratory Cross-Sectional Study. Florida Public Health Review, 13, 91-98.

Weissman, J., Preston, S., Sebekos, E., Latorre, W., Alsaif, B., Krupp, K., Darrow, W. (May 31-June 4 2016). Can an on-campus medical home improve academic performance in college students?: An exploratory cross-sectional study. American College Health Association (ACHA) Annual Meeting, San Francisco, CA; Poster Presentation.

Weissman, J., Preston, S., Sebekos, E., Latorre, W., Alsaif, B., Krupp, K., Darrow, W. (May 31-June 4 2016). Psychological health and academic success in college students: An exploratory cross-sectional study. American Public Health Association (APHA) Annual Meeting, San Francisco, CA; Special ICTHP Poster Session. 